\title{
An extremal eigenvalue problem for the Wentzell-Laplace operator
}

\author{
M. Dambrine ${ }^{\text {a }}$, D. Kateb ${ }^{\text {b }}$, J. Lamboley ${ }^{\mathrm{c}, *}$ \\ a Université de Pau et des Pays de l'Adour, France \\ ${ }^{\mathrm{b}}$ Université de Technologie de Compiègne, France \\ c CEREMADE, UMR CNRS 7534, Université Paris-Dauphine, France
}

Received 27 January 2014; received in revised form 24 October 2014; accepted 6 November 2014

Available online 18 November 2014

\begin{abstract}
We consider the question of giving an upper bound for the first nontrivial eigenvalue of the Wentzell-Laplace operator of a domain $\Omega$, involving only geometrical information. We provide such an upper bound, by generalizing Brock's inequality concerning Steklov eigenvalues, and we conjecture that balls maximize the Wentzell eigenvalue, in a suitable class of domains, which would improve our bound. To support this conjecture, we prove that balls are critical domains for the Wentzell eigenvalue, in any dimension, and that they are local maximizers in dimension 2 and 3, using an order two sensitivity analysis. We also provide some numerical evidence.
\end{abstract}

๑ 2014 L'Association Publications de l'Institut Henri Poincaré. Published by Elsevier B.V. All rights reserved.

MSC: primary 35P15; secondary 49K20, 49K40

Keywords: Wentzell eigenvalues; Eigenvalue estimates; Shape optimization; Shape derivatives; Stability; Quantitative isoperimetric inequality

\section{Introduction}

Background. Let $d \geq 2$ and $\Omega$ be a bounded domain in $\mathbb{R}^{d}$ (i.e. a bounded connected open set) supposed to be sufficiently smooth (of class $C^{3}$ ), and we denote by $\Delta_{\tau}$ the Laplace-Beltrami operator on $\partial \Omega$. Motivated by generalized impedance boundary conditions, we consider the eigenvalue problem for Wentzell boundary conditions

$$
\begin{cases}-\Delta u=0 & \text { in } \Omega \\ -\beta \Delta_{\tau} u+\partial_{n} u=\lambda u & \text { on } \partial \Omega\end{cases}
$$

where $\beta$ is a given real number and $\partial_{n}$ denotes the outward unit normal derivative.

The coefficient $\beta$ appears as a surface diffusion coefficient arising in a passage to the limit in the thickness of the boundary layer for coated object (see $[22,1,16]$ ). A general derivation of Wentzell boundary conditions can be

\footnotetext{
* Corresponding author.

E-mail addresses: marc.dambrine@univ-pau.fr (M. Dambrine), djalil.kateb@utc.fr (D. Kateb), lamboley@math.cnrs.fr (J. Lamboley).

URLs: http://web.univ-pau.fr/ mdambrin/Marc_Dambrine/Home.html (M. Dambrine), http://www.lmac.utc.fr/membres/kateb (D. Kateb), https://www.ceremade.dauphine.fr/ lamboley/ (J. Lamboley).
} 
found in [15]. The coefficient can be either positive or negative. We first consider the case $\beta \geq 0$ where the obtained boundary value problem is coercive.

This problem couples surface and volume effects through the Steklov eigenvalue problem in $\Omega$ with the LaplaceBeltrami eigenvalue problem on $\partial \Omega$. Let us recall some known facts about these two problems. The Steklov eigenvalue problem consists in solving

$$
\begin{cases}\Delta u=0 & \text { in } \Omega \\ \partial_{n} u=\lambda^{S} u & \text { on } \partial \Omega\end{cases}
$$

It has a discrete spectrum consisting of a sequence

$$
\lambda_{0}^{S}(\Omega)=0<\lambda_{1}^{S}(\Omega) \leq \lambda_{2}^{S}(\Omega) \ldots \rightarrow+\infty
$$

where the $\lambda^{S}$ are called Steklov eigenvalues. Brock-Weinstock inequality states that $\lambda_{1}^{S}$ is maximized by the ball among all open sets of fixed volume $|\Omega|$. It was first proved in the case $d=2$ by Weinstock and extended by Brock to any dimension in [6] (Weinstock inequality is slightly stronger but restricted to simply-connected domains: he proved indeed that the disk maximizes $\lambda_{1}^{S}$ among simply-connected sets of given perimeter). A quantitative form of this inequality was recently obtained by Brasco, De Philippis and Ruffini who proved in [5] that

$$
\lambda_{1}^{S}(\Omega) \leq \lambda_{1}^{S}(B)\left[1-\delta_{d}\left(\frac{\left|\Omega \Delta B\left(x_{\partial \Omega}\right)\right|}{|\Omega|}\right)^{2}\right],
$$

where $\delta_{d}$ is an explicit nonnegative constant depending only on $d, x_{\partial \Omega}$ is the center of mass of $\partial \Omega$ and $B\left(x_{\partial \Omega}\right)$ is the ball centered in $x_{\partial \Omega}$ with volume $|\Omega|{ }^{1}$ Let us emphasize that no additional topological assumption is needed.

It is well-known that the spectrum of the Laplace-Beltrami operator on $\partial \Omega$, that is numbers $\lambda$ such that the equation $-\Delta_{\tau} u=\lambda u$ on $\partial \Omega$ has nontrivial solutions, is also discrete and satisfies:

$$
\lambda_{0}^{L B}(\partial \Omega)=0<\lambda_{1}^{L B}(\partial \Omega) \leq \lambda_{2}^{L B}(\partial \Omega) \ldots \rightarrow+\infty
$$

Again, one can ask if $\lambda_{1}^{L B}$ takes its maximal value on the euclidean sphere, among hypersurfaces of fixed $(d-1)$-dimensional volume. Here, the answer is more complicated than for the Steklov problem. It depends on both the topology of the surface and the dimension. In [19], Hersch gave a positive answer if $d=3$ for surfaces homomorphic to the euclidean sphere. In the cases $d>3$ or without topological restriction, the answer is negative (see $[3,10,11]$, and Section 2.1 for the 2-dimensional case).

When $\beta \geq 0$, the spectrum of the Laplacian with Wentzell conditions consists of an increasing countable sequence of eigenvalues

$$
\lambda_{0, \beta}(\Omega)=0<\lambda_{1, \beta}(\Omega) \leq \lambda_{2, \beta}(\Omega) \ldots \rightarrow+\infty
$$

with corresponding real orthonormal (in $L^{2}(\partial \Omega)$ ) eigenfunctions $u_{0}, u_{1}, u_{2}, \ldots$ As in the previous cases, the first eigenvalue is zero with constants as corresponding eigenfunctions. As usual, we adopt the convention that each eigenvalue is repeated according to its multiplicity. Hence, the first eigenvalue of interest is $\lambda_{1, \beta}$. A variational characterization of the eigenvalues is available: we introduce the Hilbert space

$$
\mathrm{H}(\Omega)=\left\{u \in \mathrm{H}^{1}(\Omega), \operatorname{Tr}_{\partial \Omega}(u) \in \mathrm{H}^{1}(\partial \Omega)\right\},
$$

where $\operatorname{Tr}_{\partial \Omega}$ is the trace operator, and we define on $\mathrm{H}(\Omega)$ the two bilinear forms

$$
A_{\beta}(u, v)=\int_{\Omega} \nabla u \cdot \nabla v d x+\beta \int_{\partial \Omega} \nabla_{\tau} u . \nabla_{\tau} v d \sigma, \quad B(u, v)=\int_{\partial \Omega} u v,
$$

where $\nabla_{\tau}$ is the tangential gradient. Since we assume $\beta$ is nonnegative, the two bilinear forms are positive and the variational characterization for the $k$-th eigenvalue is

$$
\lambda_{k, \beta}(\Omega)=\min \left\{\frac{A_{\beta}(v, v)}{B(v, v)}, v \in \mathrm{H}(\Omega), \int_{\partial \Omega} v u_{i}=0, i=0, \ldots, k-1\right\}
$$

\footnotetext{
1 The results in [5] are stated with the Fraenkel asymmetry, meaning that the previous inequality is stated for the ball $B$ of volume $|\Omega|$ that minimizes $|\Omega \Delta B|$, but from the proof (see [5, Section 5]) we can conclude that the ball $B\left(x_{\partial \Omega}\right)$ of volume $|\Omega|$ and such that $\int_{\partial \Omega}\left(x-x_{\partial \Omega}\right) d \sigma=0$ is in fact valid as well.
} 
In particular, when $k=1$, the minimum is taken over the functions orthogonal to the eigenfunctions associated to $\lambda_{0, \beta}=0$, i.e. constant functions. To describe this spectrum, one can notice that the eigenvalue problem can be rewritten purely on $\partial \Omega$ as:

$$
-\beta \Delta_{\tau} u+\mathrm{D} u=\lambda u
$$

where D denotes the Dirichlet-to-Neumann map, that is a selfadjoint, positive pseudodifferential operator of order one. Therefore, this problem can be seen as a compact perturbation of the usual Laplace-Beltrami operator. This point of view was used in [4] where it is proven that high order eigenvalues of the Laplace-Wentzell problem look like those of the Laplace-Beltrami operator.

However, we are interested in this work, in studying low order eigenvalues and more precisely in giving an upper bound for the second eigenvalue $\lambda_{1, \beta}$ involving only geometrical information. Please remark that we are not seeking for lower bound, because even with very strong geometrical assumption, there is none. Indeed, a consequence of our results is that

$$
\inf \left\{\lambda_{1, \beta}(\Omega), \Omega \text { convex, }|\Omega|=m\right\}=0
$$

for any value of $\beta \geq 0$ and $m \geq 0$, see Remark 2.5. An important remark at this point is that the bilinear form $A_{\beta}$ is not homogeneous with respect to dilatation of the domain. Therefore, the volume of $\Omega$ plays a crucial role in $\lambda_{1, \beta}$. As a surface term appears also in $A_{\beta}$ (corresponding to the Laplace-Beltrami operator), the perimeter of $\Omega$ (i.e. the volume of $\partial \Omega$ ) should also play a crucial role.

Notice that when $\beta=0$ we retrieve the Steklov eigenvalues, and we recover the Laplace-Beltrami eigenvalues by considering $\frac{1}{\beta} \lambda_{1, \beta}$ and letting $\beta$ go to $+\infty$, see Section 2.1.

Note also that the close but distinct eigenvalue problem

$$
\begin{cases}-\Delta u=\lambda u & \text { in } \Omega \\ \Delta u+\alpha \partial_{n} u+\gamma u=0 & \text { on } \partial \Omega\end{cases}
$$

was considered by J.B. Kennedy in [21]. He transforms this problem into a Robin type problem to prove a Faber-Krahn type inequality when the constants $\alpha, \gamma$ are nonnegative: the ball is the best possible domain among those of given volume.

The results of the paper. We first apply the strategy of F. Brock for the Steklov eigenvalue problem to the Wentzell eigenvalue problem and obtain a first upper bound of $\lambda_{1, \beta}(\Omega)$ in terms of purely geometric quantities (we actually provide a refined version, using [5]):

Theorem 1.1. Let $\Omega$ be a smooth set such that $\int_{\partial \Omega} x=0$. Let $\Lambda[\Omega]$ be the spectral radius of the symmetric and positive semidefinite matrix $P(\Omega)=\left(p_{i j}\right)_{i, j=1, \ldots, d}$ defined as

$$
p_{i j}=\int_{\partial \Omega}\left(\delta_{i j}-n_{i} n_{j}\right)
$$

where $\boldsymbol{n}$ is the outward normal vector to $\partial \Omega$. Then if $\beta \geq 0$, one has:

$$
S(\Omega):=\sum_{i=1}^{d} \frac{1}{\lambda_{i, \beta}(\Omega)} \geq \frac{\int_{\partial \Omega}|x|^{2}}{|\Omega|+\beta \Lambda[\Omega]} \geq \frac{d \omega_{d}^{-1 / d}|\Omega|^{\frac{d+1}{d}}}{|\Omega|+\beta \Lambda[\Omega]}\left[1+\gamma_{d}\left(\frac{|\Omega \Delta B|}{|B|}\right)^{2}\right],
$$

where

$$
\gamma_{d}=\frac{d+1}{d} \frac{2^{1 / d}-1}{4},
$$

$\omega_{d}=\left|B_{1}\right|$ and $B$ is the ball of volume $|\Omega|$ and centered at 0 . Equality holds in (9) if $\Omega$ is a ball.

A consequence of Theorem 1.1 is the following upper bound for $\lambda_{1, \beta}(\Omega)$. 
Corollary 1.2. With the same notations as in Theorem 1.1, if $\beta \geq 0$, it holds:

$$
\lambda_{1, \beta}(\Omega) \leq d \frac{|\Omega|+\beta \Lambda[\Omega]}{\int_{\partial \Omega}|x|^{2}} \leq \frac{|\Omega|+\beta \Lambda[\Omega]}{\omega_{d}^{-1 / d}|\Omega|^{\frac{d+1}{d}}\left[1+\gamma_{d}\left(\frac{|\Omega \Delta B|}{|B|}\right)^{2}\right]},
$$

where $B$ and $\gamma_{d}$ are as in Theorem 1.1. Equality holds in (11) if $\Omega$ is a ball.

Note that the method used for the Wentzell eigenvalue problem also applies for the Laplace-Beltrami case and provides an upper bound for $\lambda_{1}^{L B}$ without any topological assumption on $\Omega$.

Theorem 1.3. With the same notations as in Theorem 1.1, it holds

$$
S^{L B}(\partial \Omega):=\sum_{i=1}^{d} \frac{1}{\lambda_{i}^{L B}(\partial \Omega)} \geq \frac{\int_{\partial \Omega}|x|^{2}}{\Lambda[\Omega]} \geq \frac{d \omega_{d}^{-1 / d}|\Omega|^{\frac{d+1}{d}}}{\Lambda[\Omega]}\left[1+\gamma_{d}\left(\frac{|\Omega \Delta B|}{|B|}\right)^{2}\right]
$$

and

$$
\lambda_{1}^{L B}(\partial \Omega) \leq d \frac{\Lambda[\Omega]}{\int_{\partial \Omega}|x|^{2}} \leq \frac{\Lambda[\Omega]}{\omega_{d}^{-1 / d}|\Omega|^{\frac{d+1}{d}}\left[1+\gamma_{d}\left(\frac{|\Omega \Delta B|}{|B|}\right)^{2}\right]} .
$$

Equality holds in (12) and (13) if $\Omega$ is a ball.

It is expected in this type of extremal eigenvalue problem that balls are maximizers. We are not able to fully justify the natural following conjecture:

Conjecture. The ball maximizes the first non-trivial Wentzell-Laplace eigenvalue among smooth open sets of given volume and which are homeomorphic to the ball.

The topological restriction is motivated by the limit case $\beta \rightarrow+\infty$ as we noticed before (see also Section 2.1). In Section 2.2, we observe that the intermediate bound in (11) has both its numerator and denominator that are minimized by the ball, under volume constraint, so there is a competition. In Section 2.3 we observe that in fact, the ball does not minimize this bound in general (see Fig. 1). Therefore, we cannot deduce from this bound the maximality of balls (though it might work for certain values of $\beta$ and the volume constraint). About the upper bound (11), we show that it is larger than $\lambda_{1, \beta}(B)$ for every $\beta>0$ (with equality for the ball) and hence again does not imply that balls are maximizing $\lambda_{1, \beta}$. To check if balls are relevant candidates for maximizers in our case, we then turn our attention to a shape sensitivity analysis of $\lambda_{1, \beta}$.

Therefore, we first wonder if the ball is a critical shape in any dimension. With respect to shape sensitivity, the main difficulty is to handle multiple eigenvalues which leads to a nonsmooth dependency of $\lambda_{1, \beta}$ with respect to $\Omega$. However, for a fixed deformation field $\boldsymbol{V} \in W^{3, \infty}\left(\Omega, \mathbb{R}^{d}\right)$, along the transport of $\Omega$ by $T_{t}=I+t \boldsymbol{V}$, we prove the existence of smooth branches of eigenvalues and eigenfunctions associated to the subspace generated by the group of eigenvalues and provide a characterization of the derivative along the branches: $\lambda_{1, \beta}$ is then the minimum value among these $d$ smooth branches.

Theorem 1.4. We distinguish the case of simple and multiple eigenvalue.

- If $\lambda=\lambda_{k, \beta}(\Omega)$ is a simple eigenvalue of the Wentzell problem, then the application $t \mapsto \lambda(t)=\lambda_{k, \beta}\left(\Omega_{t}\right)$ (where $\left.\Omega_{t}=(I+t \boldsymbol{V})(\Omega)\right)$ is differentiable and the derivative at $t=0$ is

$$
\lambda^{\prime}(0)=\int_{\partial \Omega} V_{n}\left(\left|\nabla_{\tau} u\right|^{2}-\left|\partial_{n} u\right|^{2}-\lambda H\left|u_{0}\right|^{2}+\beta\left(H I_{d}-2 D^{2} b\right) \nabla_{\tau} u . \nabla_{\tau} u\right) d \sigma
$$


where $u$ is the normalized eigenfunction associated to $\lambda, D^{2} b$ is the Hessian of the signed distance function (see (48)), $H=\operatorname{Tr}\left(D^{2} b\right)$ is the mean curvature of $\partial \Omega, I_{d}$ is the identity matrix of size $d$, and $V_{n}=\boldsymbol{V} \cdot \mathbf{n}_{\partial \Omega}$ is the normal component of the deformation. Moreover, the shape derivative $u^{\prime}$ at $t=0$ of the eigenfunction satisfies

$$
\left\{\begin{aligned}
& \Delta u^{\prime}=0 \quad \text { in } \Omega, \\
&-\beta \Delta_{\tau} u^{\prime}+\partial_{n} u^{\prime}-\lambda u^{\prime}= \beta \Delta_{\tau}\left(V_{n} \partial_{n} u\right)+\beta \operatorname{div}_{\tau}\left(V_{n}\left(H I_{d}-2 D^{2} b\right) \nabla_{\tau} u\right) \\
&+\operatorname{div}_{\tau}\left(V_{n} \nabla_{\tau} u\right)-\lambda^{\prime} u+\lambda V_{n}\left(\partial_{n} u+H u\right) \quad \text { on } \partial \Omega .
\end{aligned}\right.
$$

- Let $\lambda$ be a multiple eigenvalue of order $m \geq 2$. Let $\left(u_{k}\right)_{k=1, \ldots, m}$ denote the eigenfunctions associated to $\lambda$. Then there exist $m$ functions $t \mapsto \lambda_{k, \beta}(t), k=1, \ldots, m$, defined in a neighborhood of 0 such that

$-\lambda_{k, \beta}(0)=\lambda$,

- for every $t$ in a neighborhood of $0, \lambda_{k, \beta}(t)$ is an eigenvalue of $\Omega_{t}=(I+t \boldsymbol{V})(\Omega)$,

- the functions $t \mapsto \lambda_{k, \beta}(t), k=1, \ldots, m$, admit derivatives and their values at 0 are the eigenvalues of the $m \times m$ matrix $M=M_{\Omega}\left(V_{n}\right)$ of entries $\left(M_{i j}\right)$ defined by

$$
M_{i j}=\int_{\partial \Omega} V_{n}\left(\nabla_{\tau} u_{i} . \nabla_{\tau} u_{j}-\partial_{n} u_{i} \partial_{n} u_{j}-\lambda H u_{i} u_{j}+\beta\left(H I_{d}-2 D^{2} b\right) \nabla_{\tau} u_{i} . \nabla_{\tau} u_{j}\right) d \sigma .
$$

Notice that in the notations above and contrary to (3), the functions $\lambda_{k}(t)$ are no longer ordered. As a byproduct of this result, notice that we can write the corresponding shape derivatives for the Steklov and Laplace-Beltrami eigenvalue problem (see Appendix E). Another consequence of this result, regarding our conjecture, is that we are able to check that balls are critical shapes for $\lambda_{1, \beta}$ by computing the trace of the previously defined matrix $M=M_{B}$ (recall that $\lambda_{1, \beta}(B)$ is an eigenvalue of multiplicity $d$, the dimension of the ambient space). But first, we make a short remark about the notion of volume preserving deformation:

Remark 1.5. In the next results and in many places in the paper, we will consider volume preserving smooth deformations of domains, that is to say $\Omega_{t}=T_{t}(\Omega)$ where $t \mapsto T_{t}$ satisfies:

- $T_{0}=I d$,

- for every $t$ near $0, T_{t}$ is a $W^{3, \infty}$-diffeomorphism from $\Omega$ onto its image $\Omega_{t}=T_{t}(\Omega)$,

- the application $t \mapsto T_{t}$ is real-analytic near $t=0$,

- for every $t$ near $0,\left|\Omega_{t}\right|=|\Omega|$.

More generally, it can be sufficient to assume that the volume is preserved at the first or the second order, depending on whether we are interested in first or second order conditions. For example, if one considers $T_{t}=I+t \boldsymbol{V}$ the vector field $\boldsymbol{V}$ is said to be volume preserving at first order if it satisfies $\int_{\partial \Omega} V_{n} d \sigma=0$; indeed for $\Omega_{t}=(I+t \boldsymbol{V})(\Omega)$, we have $\frac{d}{d t}{ }_{t=0}\left|\Omega_{t}\right|=\int_{\partial \Omega} V_{n} d \sigma$.

When dealing with second order considerations as in Theorem 1.7, we need that the volume is preserved at the second order, so $T_{t}$ is volume preserving at second order if

$$
\left.\frac{d^{2}}{d t^{2}}\left|\Omega_{t}\right|\right|_{t=0}=\int_{\partial \Omega}\left(W+V_{n} \partial_{n} V_{n}+H V_{n}^{2}\right) d \sigma=0,
$$

where $\boldsymbol{V}=\frac{1}{t}\left(T_{t}-I\right), V_{n}$ is the value at $t=0$ of $\boldsymbol{V} \cdot n_{\partial \Omega_{t}}$, and $W$ denotes the derivative of $\boldsymbol{V} \cdot n_{\partial \Omega_{t}}$ with respect to $t$ at $t=0$.

Proposition 1.6. Any ball $B$ is a critical shape for $\lambda_{1, \beta}$ with volume constraint, in the sense that for every volume preserving deformations $\boldsymbol{V}$,

$$
\operatorname{Tr}\left(M_{B}\left(V_{n}\right)\right)=\sum_{k=1}^{d} \lambda_{k, \beta}^{\prime}(0)=0,
$$

where $\left(t \mapsto \lambda_{k, \beta}(t)\right)_{k=1 \ldots d}$ are defined in Theorem 1.4. 
In particular, $0 \in \partial \lambda_{1, \beta}\left(B ; V_{n}\right):=\left[\inf _{i=1 \cdots d} \lambda_{i, \beta}^{\prime}(0), \sup _{i=1 \cdots d} \lambda_{i, \beta}^{\prime}(0)\right]$ the directional subdifferential associated to the first nontrivial eigenvalue.

Moreover, this subdifferential reduces to $\{0\}$ if $V_{n}$ is orthogonal to spherical harmonics of order two: in other words, in that case, the directional derivative exists in the usual sense and vanishes.

Two situations can now occur: either the subdifferential in direction $V_{n}$ is not reduced to $\{0\}$ and then one can deduce from the previous statement that $B$ locally maximizes $\lambda_{1, \beta}$ along $t \mapsto B_{t}$ (see for example (c) and (d) in Fig. 5), or the subdifferential in direction $V_{n}$ is $\{0\}$ and then this first order shape calculus does not allow us to conclude that the ball is a local maximizer of $\lambda_{1, \beta}$. Hence, for the directions $V_{n}$ in $\mathcal{H}$ defined as the Hilbert space generated by spherical harmonics of order greater or equal to three, we now consider the second order analysis to wonder if the ball satisfies the second order necessary condition of optimality, and obtain the following result in dimension two and three.

Theorem 1.7. Let $B$ be a ball of radius $R$ in $\mathbb{R}^{2}$ or $\mathbb{R}^{3}$ (i.e. $d=2$ or $d=3$ ) and $t \mapsto B_{t}=T_{t}(B)$ a second order volume preserving deformation. $\lambda_{1, \beta}(B)$ is an eigenvalue of multiplicity $d$, the dimension, and we denote by $t \mapsto \lambda_{k, \beta}(t)$, $k=1, \ldots, d$, the branches obtained in Theorem 1.4.

Then the functions $t \mapsto \lambda_{k, \beta}(t), k=1, \ldots, d$, admit a second derivative and their values at 0 are the eigenvalues of the $d \times d$ matrix $E=E_{B}\left(V_{n}\right)$ defined in Section 4. Moreover, there exists a nonnegative number $\mu(=\mu(\beta))$ independent of the radius $R$ such that

$$
\operatorname{Tr}\left(E_{B}\left(V_{n}\right)\right)=\sum_{k=1}^{d} \lambda_{k, \beta}^{\prime \prime}(0) \leq-\mu K(R) \int_{\partial B}\left(\left|\nabla_{\tau} V_{n}\right|^{2}+\left|V_{n}\right|^{2}\right) d \sigma=-\mu K(R)\left\|V_{n}\right\|_{\mathrm{H}^{1}(\partial B)}^{2}
$$

holds for all $V_{n} \in \mathcal{H}$, with $K(R)=\frac{d}{R^{2+d} \omega_{d-1}}$.

As a consequence of Proposition 1.6 and Theorem 1.7, we have the result:

Corollary 1.8. If $B$ is a ball in $\mathbb{R}^{2}$ or $\mathbb{R}^{3}$, and $t \mapsto T_{t} \in W^{3, \infty}\left(B, \mathbb{R}^{d}\right)$ a smooth (second order) volume preserving deformation, then

$$
\lambda_{1, \beta}(B) \geq \lambda_{1, \beta}\left(T_{t}(B)\right), \quad \text { for } t \text { small enough. }
$$

Plan of the paper. The paper is organized as follows: in Section 2, we prove Theorem 1.1 by adapting the strategy of Brock and present some numerical tests to illustrate the sharpness of the upper bound. The first order shape analysis is presented in Section 3, while the second order shape analysis is presented in Section 4. The background material for shape calculus and the proofs of technical intermediary results are postponed to the annexes.

\section{Upper bound for $\lambda_{1, \beta}$}

\subsection{Preliminary remarks and results}

Let us start by a few remarks on the proofs in the two limit cases $\beta \rightarrow+\infty$ (that is the Laplace-Beltrami eigenvalue problem), and $\beta=0$ (that is the Steklov eigenvalue problem).

On the Laplace-Beltrami case: The case $d=2$ is trivial: it suffices to argue on each connected component of $\partial \Omega$. We introduce $\gamma:[0, L]$ a parametrization by the arclength of a connected component $\Gamma$ of $\partial \Omega$, then for any $u \in \mathrm{H}^{1}(\partial \Omega)$, the Rayleigh quotient can be written as

$$
\frac{\int_{\Gamma}\left|\nabla_{\tau} u\right|^{2}}{\int_{\Gamma} u^{2}}=\frac{\int_{0}^{L}\left[(u \circ \gamma)^{\prime}\right]^{2}}{\int_{0}^{L}(u \circ \gamma)^{2}} .
$$

Hence, the $\lambda_{1}^{L B}(\Gamma)$ is nothing but the infimum of $\left\|u^{\prime}\right\|_{\mathrm{L}^{2}(0, L)}^{2}$ among periodic functions $u$ with 0 mean value and $\|u\|_{\mathrm{L}^{2}(0, L)}=1$, that is to say $4 \pi^{2} / L^{2}$. It is a decreasing function of the length of the connected component of the 
boundary. Then, if $\Omega$ is simply connected, combined with the isoperimetric inequality, the previous computations lead to $\lambda_{1}^{L B}(\partial \Omega) \leq \lambda_{1}^{L B}(\partial B)$ where $B$ is a disk of the same area as $\Omega$.

Moreover, if $\partial \Omega$ has more than one connected component, then $\lambda_{1}^{L B}=0$ since the multiplicity of 0 as eigenvalue is at least the number of connected component. To check that claim, it suffices to check that the functions taking the value 1 on one of the connected component and 0 elsewhere are independent eigenfunctions associated to the eigenvalue 0 . We conclude that in dimension $2, \lambda_{1}^{L B}(\partial \Omega) \leq \lambda_{1}^{L B}(\partial B)$, where $B$ is a disk of the same area as $\Omega$.

The case $d=3$ is more complex. There is a classical result of J. Hersch [19]: if $\Omega \subset \mathbb{R}^{3}$ is homeomorphic to the ball, then

$$
\lambda_{1}^{L B}(\partial \Omega) \leq \lambda_{1}^{L B}(\partial B), \quad \text { for all } \Omega \text { such that }|\partial \Omega|=|\partial B| .
$$

We first extend Hersch statement to domains of the same volume by a classical homogeneity argument.

Lemma 2.1. If $\Omega \subset \mathbb{R}^{3}$ is homeomorphic to the ball, then

$$
\lambda_{1}^{L B}(\partial \Omega) \leq \lambda_{1}^{L B}(\partial B) \quad \text { if }|\Omega|=|B| .
$$

Proof. One easily checks that $\Omega \mapsto \lambda_{1}^{L B}(\partial \Omega)$ is homogeneous of degree -2 , so $\Omega \mapsto \lambda_{1}^{L B}(\Omega)|\partial \Omega|^{2 /(d-1)}$ is homogeneous of degree 0 . Then we get from Hersch's inequality (15), that

$$
\lambda_{1}^{L B}(\partial \Omega)|\partial \Omega|^{\frac{2}{d-1}} \leq \lambda_{1}^{L B}(\partial B)|\partial B|^{\frac{2}{d-1}}, \quad \text { for all } \Omega \text { such that }|\partial \Omega|=|\partial B| .
$$

Thanks to the invariance by translation of $\lambda_{1}^{L B}$ and the perimeter, and using the 0 -homogeneity of the previous product, we get that the previous inequality is in fact valid for any ball $B$ and any domain $\Omega$. We combine with the isoperimetric inequality

$$
\frac{|\partial B|^{\frac{d}{d-1}}}{|B|} \leq \frac{|\partial \Omega|^{\frac{d}{d-1}}}{|\Omega|}
$$

to conclude.

On the Steklov case: In the general case $\beta \geq 0$, we will adapt the original Brock's proof; the main tool is an isoperimetric inequality for the moment of inertia of the boundary $\partial \Omega$ with respect to the origin. The general form of the weighted isoperimetric inequality due to F. Betta, F. Brock, A. Mercaldo and M.R. Posteraro [2] is:

Lemma 2.2. Let $\Omega \subset \mathbb{R}^{d}$ be an open set and let $f$ be a continuous, nonnegative and nondecreasing function defined on $[0, \infty]$. Moreover, we suppose that

$$
t \mapsto\left(f\left(t^{\frac{1}{d}}\right)-f(0)\right) t^{1-\frac{1}{d}} \quad \text { is convex for } t \geq 0
$$

Then

$$
\int_{\partial \Omega} f(|x|) d \sigma \geq f(R)\left|\partial B_{R}\right|,
$$

where $B_{R}$ is the ball centered at the origin such that $\left|B_{R}\right|=|\Omega|$.

Let us remark that the function $t \mapsto t^{p}$ satisfies the assumptions of the lemma as soon as $p \geq 1$ and in particular for $p=2$. In that case and in order to prove a refinement of Brock's inequality, L. Brasco, G. De Philippis and B. Ruffini established a qualitative refinement of this inequality (Theorem B of [5]):

Lemma 2.3. There exists an explicit dimensional constant $\gamma_{d}$ such that for every bounded, open Lipschitz set $\Omega$ in $\mathbb{R}^{d}$,

$$
\int_{\partial \Omega}|x|^{2} d \sigma \geq R^{2}\left|\partial B_{R}\right|\left[1+\gamma_{d}\left(\frac{\left|\Omega \Delta B_{R}\right|}{\left|B_{R}\right|}\right)^{2}\right],
$$

where $B_{R}$ is the ball centered at the origin such that $\left|B_{R}\right|=|\Omega|$ and $\gamma_{d}$ is the constant defined in (10). 
On the Wentzell case: An important remark for the sequel is the particular case when $\Omega$ is a ball $B_{R}$ of radius $R$. The eigenspace corresponding to $\lambda_{1, \beta}$ is $d$-dimensional: it consists of the restrictions on the sphere $S_{R}^{d-1}$ of the linear functions in $\mathbb{R}^{d}$ spanned by the coordinate functions. It follows, from the theory of spherical harmonic functions that

$$
\left.\lambda_{1, \beta}\left(B_{R}\right)=\lambda_{2, \beta}\left(B_{R}\right)=\ldots=\lambda_{d, \beta} B_{R}\right)=\frac{(d-1) \beta+R}{R^{2}} .
$$

The Laplace-Beltrami operator on $\partial B_{R}$ and the Steklov operator also are diagonal on the basis of spherical harmonics, hence

$$
\lambda_{1, \beta}\left(B_{R}\right)=\lambda_{1}^{S}\left(B_{R}\right)+\beta \lambda_{1}^{L B}\left(\partial B_{R}\right),
$$

and more generally the eigenvalue associated to spherical harmonics of order $l$ is

$$
\lambda_{(l)}\left(B_{R}\right)=\frac{l(l+d-2) \beta+R}{R^{2}} .
$$

But, this situation is specific to the ball: indeed, in general we only have the inequality

$$
\lambda_{1, \beta}(\Omega) \geq \lambda_{1}^{S}(\Omega)+\beta \lambda_{1}^{L B}(\Omega) .
$$

Moreover, we can easily prove that for any smooth $\Omega, \lim _{\beta \rightarrow \infty} \frac{1}{\beta} \lambda_{1, \beta}(\Omega)=\lambda_{1}^{L B}(\Omega)$ : indeed, we have a first trivial inequality $\frac{1}{\beta} \lambda_{1, \beta}(\Omega) \geq \lambda_{1}^{L B}(\Omega)$ for any $\beta \geq 0$, and using the variational formulation (5), we obtain $\forall v \in \mathrm{H}(\Omega)$ with the additional condition $\int_{\partial \Omega} v=0$,

$$
\varlimsup_{\beta \rightarrow \infty} \frac{1}{\beta} \lambda_{1, \beta}(\Omega) \leq \varlimsup_{\beta \rightarrow \infty} \frac{\frac{1}{\beta} \int_{\Omega}|\nabla v|^{2}+\int_{\partial \Omega}\left|\nabla_{\tau} v\right|^{2}}{\int_{\partial \Omega} v^{2}}=\frac{\int_{\partial \Omega}\left|\nabla_{\tau} v\right|^{2}}{\int_{\partial \Omega} v^{2}}
$$

which leads to the result.

For example if $d=3$, combining Brock's inequality and Lemma 2.1, we obtain that the right-hand side in the previous inequality is maximized by the ball, among domains of given volume and homeomorphic to the ball. Unfortunately, this is not enough to obtain that balls are maximizing the Wentzell eigenvalue.

So in order to obtain an estimate of $\lambda_{1, \beta}$, we look at the strategies used for the extremal problems, which are the Steklov $(\beta=0)$ and the Laplace-Beltrami $(\beta \rightarrow+\infty)$ cases. The strategies of Brock and Hersch for those cases are actually close but distinct: they use the coordinate functions as test functions in the Rayleigh quotient characterization of eigenvalues. In the case of the Laplace-Beltrami operator though, J. Hersch had an additional step: he first transports the surface $\partial \Omega$ on the sphere by a conformal mapping, and uses the conformal invariance of the Dirichlet energy for 2-dimensional surfaces. In the following, we choose to follow the ideas of Brock. This allows to obtain an estimate with no assumption on the topology or the dimension of the domain. Indeed, the above mentioned phenomenon of decoupling between the different connected components does not appear in the Steklov case, due to the volume term, and in fact Brock's result is valid for every (smooth enough) domain. The same volume term appears in the Wentzell case and the approach of Brock is then the natural one. However, one expects from these topological considerations that it will not provide an optimal result.

\subsection{Proof of Theorem 1.1}

Our strategy to prove Theorem 1.1 is to use the following characterization for the inverse trace of eigenvalues (stated by J. Hersch in [18] and proved by G. Hile and Z. Xu in [20])

$$
\sum_{i=1}^{d} \frac{1}{\lambda_{i, \beta}}=\max _{v_{1}, \cdots, v_{d}} \sum_{i=1}^{d} \frac{B\left(v_{i}, v_{i}\right)}{A_{\beta}\left(v_{i}, v_{i}\right)}
$$

where the functions $\left(v_{i}\right)_{i=1, \ldots, d}$ are nonzero functions that are $B$-orthogonal to the constants and pairwise $A_{\beta}$-orthogonal.

Before proving Theorem 1.1, we now present some preliminary results. 
Lemma 2.4. The matrix $P[\Omega]$ defined by (8) is symmetric, positive definite. Its spectral radius $\Lambda[\Omega]$ satisfies

$$
(d-1)|\partial \Omega| \geq \Lambda[\Omega] \geq \frac{d-1}{d}|\partial \Omega| .
$$

In particular, among sets of given volume, the spectral radius is minimal for the ball.

Proof. The matrix $P(\Omega)$ is symmetric by definition. For $\mathbf{y}=\left(y_{1}, \cdots, y_{d}\right) \in \mathbb{R}^{d}$ with $\mathbf{y} \neq \mathbf{0}$, we check that

$$
\sum_{i, j=1}^{d} y_{i}\left(\delta_{i j}-\boldsymbol{n}_{i} \boldsymbol{n}_{j}\right) y_{j}=\mathbf{y}^{T} \mathbf{y}-\left(\mathbf{y}^{T} \boldsymbol{n}\right)^{2} \geq 0
$$

by Cauchy-Schwarz inequality. By integration over $\partial \Omega, P[\Omega]$ is positive semidefinite. Assume, by contradiction, that $P$ is not definite: then there is a vector $\mathbf{y} \neq \mathbf{0}$ such that

$$
0=\sum_{i, j=1}^{d} y_{i}\left(\int_{\partial \Omega}\left(\delta_{i j}-\boldsymbol{n}_{i} \boldsymbol{n}_{j}\right)\right) y_{j}=\int_{\partial \Omega}\left(\mathbf{y}^{T} \mathbf{y}-\left(\mathbf{y}^{T} \boldsymbol{n}\right)^{2}\right) .
$$

The equality case of Cauchy-Schwarz inequality $\mathbf{y}^{T} \mathbf{y}-\left(\mathbf{y}^{T} \boldsymbol{n}\right)^{2}=0$ is therefore satisfied everywhere on $\partial \Omega$, this holds if and only if $\mathbf{y}$ and $\boldsymbol{n}$ are colinear. Hence, $\boldsymbol{n}$ is constant on $\partial \Omega$ which contradicts the boundedness of $\Omega$.

The matrix $P[\Omega]$ has positive eigenvalues. Their sum is the trace $\operatorname{Tr}(P[\Omega])$, hence

$$
\operatorname{Tr}(P[\Omega]) \geq \Lambda[\Omega] \geq \frac{\operatorname{Tr}(P[\Omega])}{d} \quad \text { with } \operatorname{Tr}(P[\Omega])=\sum_{i=1}^{d} \int_{\partial \Omega}\left(1-\boldsymbol{n}_{i}^{2}\right)=(d-1)|\partial \Omega| .
$$

Therefore

$$
(d-1)|\partial \Omega| \geq \Lambda[\Omega] \geq \frac{(d-1)}{d}|\partial \Omega| \geq \frac{(d-1)}{d}|\partial B| .
$$

The last inequality is obtained by the usual isoperimetric inequality and assuming $B$ is a ball such that $|\Omega|=|B|$. Let us compute $\Lambda[B]$. From the invariance by rotation of the ball, there exists a real number $a$ such that $P[B]=a I_{d}$. In others words, we have

$$
\int_{\partial B} \boldsymbol{n}_{i} \boldsymbol{n}_{j}=0, \quad i \neq j \quad \text { and } \int_{\partial B}\left(1-\boldsymbol{n}_{i}^{2}\right)=\int_{\partial B}\left(1-\boldsymbol{n}_{1}^{2}\right), \quad i=1, \ldots, d .
$$

The real number $a$ is determined using the trace of the matrix: we obtain that $d \Lambda[B]=(d-1)|\partial B|$, and so $\Lambda(\Omega) \geq$ $\Lambda(B)$.

Remark 2.5. The inequalities in (22) are sharp. The lower bound is reached when $\Omega$ is a ball and the upper bound is the limit of the collapsing stadium $S_{\varepsilon}$ (union of a rectangle and two half-disks) of unit area and width $\varepsilon$ when $\varepsilon$ tends to 0 : one checks by an explicit elementary calculus that:

$$
\left|\partial S_{\varepsilon}\right|=\frac{2}{\varepsilon}+\frac{\pi \varepsilon}{2} \quad \text { while } \Lambda\left[S_{\varepsilon}\right]=\frac{2}{\varepsilon} .
$$

This example is also useful to prove (6): indeed, we easily prove

$$
\int_{\partial S_{\varepsilon}}|x|^{2} \geq \frac{\alpha}{\varepsilon^{3}}
$$

where $\alpha$ is a universal constant, so using (11), we obtain (6) for $d=2$ and $m=1$. The other cases can be handled similarly.

Proof of Theorem 1.1. We first translate and rotate coordinates $x_{i}, i=1,2, \ldots d$, such that

$$
\forall i \neq j \in \llbracket 1, d \rrbracket^{2}, \quad \int_{\partial \Omega} x_{i}=0 \quad \text { and } \quad \int_{\partial \Omega} x_{i} x_{j}=0 .
$$


We now construct a family which is pairwise $A_{\beta}$-orthogonal, and $B$-orthogonal to $\mathbb{R}$. We consider a collection of a family of functions $w_{1}, w_{2}, \ldots, w_{d}$ in the vector space spanned by the coordinate functions: there is a matrix $C$ such that

$$
w_{i}=\sum_{j=1}^{d} c_{i j} x_{j}, \quad i \in \llbracket 1, d \rrbracket .
$$

Brock used directly the coordinate functions to deal with $A_{0}$. Here, we need an $A_{\beta}$-orthogonal family, hence the matrix $C$ will be chosen to that end. Since the coordinate functions are $\mathrm{L}^{2}$ orthogonal to the constants, each $w_{i}$ is $\mathrm{L}^{2}$-orthogonal to the constants (that is to say the eigenfunctions associated to the smallest eigenvalue $\lambda_{0}=0$ ).

Let us compute $A_{\beta}\left(w_{i}, w_{j}\right)$. First, we get $\nabla w_{i}=\left(c_{i 1}, c_{i 2}, \ldots, c_{i d}\right)^{T}$ then

$$
\int_{\Omega} \nabla w_{i} \cdot \nabla w_{j}=\int_{\Omega} \sum_{k, m=1}^{d} c_{i k} c_{j m}=|\Omega|\left(C C^{T}\right)_{i j} .
$$

To compute the second term of the sum occurring in $A_{\beta}$, we recall that

$$
\nabla_{\tau} w_{i} \cdot \nabla_{\tau} w_{j}=\nabla w_{i} \cdot \nabla w_{j}-\left(\nabla w_{i} \cdot \boldsymbol{n}\right)\left(\nabla w_{j} \cdot \boldsymbol{n}_{j}\right) .
$$

We therefore get

$$
\begin{aligned}
\int_{\partial \Omega} \nabla_{\tau} w_{i} \cdot \nabla_{\tau} w_{i} & =\int_{\partial \Omega}\left[\sum_{k=1}^{d} c_{i k} c_{j k}-\left(\sum_{k=1}^{d} c_{i k} \boldsymbol{n}_{k}\right)\left(\sum_{k=1}^{d} c_{j k} \boldsymbol{n}_{k}\right)\right] \\
& =\int_{\partial \Omega}\left[\sum_{k=1}^{d} c_{i k} c_{j k}-\sum_{k, l=1}^{d} c_{i k} c_{j l} \boldsymbol{n}_{k} \boldsymbol{n}_{l}\right] .
\end{aligned}
$$

We introduce $P[\Omega]$ the matrix defined in (8) to get

$$
\int_{\partial \Omega} \nabla_{\tau} w_{i} \cdot \nabla_{\tau} w_{j}=\sum_{k, m} c_{i k} p_{k m} c_{j m}=\left(C P[\Omega] C^{T}\right)_{i j} .
$$

Gathering all the terms, it comes that

$$
A_{\beta}\left(w_{i}, w_{j}\right)=|\Omega|\left(C C^{T}\right)_{i j}+\beta\left(C P[\Omega] C^{T}\right)_{i j}
$$

Since $P[\Omega]$ is a real symmetric matrix, we can choose an orthogonal matrix $C$ such that $C P[\Omega] C^{T}$ is diagonal. Hence, $C C^{T}=I$ and finally $w_{i}$ and $w_{j}$ are $A_{\beta}$-orthogonal if $i \neq j$ while

$$
A_{\beta}\left(w_{i}, w_{i}\right)=|\Omega|+\beta\left(C P[\Omega] C^{T}\right)_{i i} \leq|\Omega|+\beta \Lambda[\Omega]
$$

and we can apply Hile and Xu's inequality (see [20]).

Since by assumption

$$
\int_{\partial \Omega} x_{i} x_{j}=0
$$

when $i \neq j$, it comes that

$$
B\left(w_{i}, w_{i}\right)=\sum_{k=1}^{d} c_{i k}^{2} \int_{\partial \Omega} x_{k}^{2}
$$

and then

$$
S(\Omega)=\sum_{i=1}^{d} \frac{1}{\lambda_{i, \beta}(\Omega)} \geq \frac{\sum_{i=1}^{d} \sum_{k=1}^{d} c_{i k}^{2} \int_{\partial \Omega} x_{k}^{2}}{|\Omega|+\beta \Lambda[\Omega]}=\frac{\sum_{k=1}^{d}\left(\int_{\partial \Omega} x_{k}^{2}\right) \sum_{i=1}^{d} c_{i k}^{2}}{|\Omega|+\beta \Lambda[\Omega]}=\frac{\int_{\partial \Omega}|x|^{2}}{|\Omega|+\beta \Lambda[\Omega]},
$$


which is the first part of the result. Then using first the isoperimetric weighted inequality (17) for $p=2$, we get

$$
\int_{\partial \Omega}|x|^{2} \geq R^{2}\left|\partial B_{R}\right|
$$

and so

$$
\frac{\int_{\partial \Omega}|x|^{2}}{|\Omega|+\beta \Lambda[\Omega]} \geq \frac{R^{2}\left|\partial B_{R}\right|}{|\Omega|+\beta \Lambda[\Omega]}=\frac{R^{2}}{\frac{\left|B_{R}\right|}{\left|\partial B_{R}\right|}+\frac{\beta \Lambda[\Omega]}{\left|\partial B_{R}\right|}} .
$$

If $\Omega=B_{R}$, we know that $d\left|B_{R}\right|=R\left|\partial B_{R}\right|$ and then

$$
\frac{R^{2}}{\frac{\left|B_{R}\right|}{\left|\partial B_{R}\right|}+\frac{\beta \Lambda\left[B_{R}\right]}{\left|\partial B_{R}\right|}}=\frac{R^{2}}{\frac{R}{d}+\beta \frac{d-1}{d}}=\frac{d}{\lambda_{1, \beta}\left(B_{R}\right)},
$$

and prove the equality case. By the quantitative version of the isoperimetric inequality for the moment of inertia of $\partial \Omega$ with respect to the origin (18), we also get the precise version:

$$
\frac{\int_{\partial \Omega}|x|^{2}}{|\Omega|+\beta \Lambda[\Omega]} \geq \frac{R^{2}\left|\partial B_{R}\right|}{|\Omega|+\beta \Lambda[\Omega]}\left[1+\gamma_{d}\left(\frac{\left|\Omega \Delta B_{R}\right|}{\left|B_{R}\right|}\right)^{2}\right] .
$$

Using the definition of $R$ and $|\Omega|=\left|B_{R}\right|$, we obtain $R^{2}\left|\partial B_{R}\right|=d \omega_{d}^{-1 / d}|\Omega|^{\frac{d+1}{d}}$ and the desired inequality.

Proof of Corollary 1.2. Since $\lambda_{1, \beta}(\Omega) \leq \lambda_{i, \beta}(\Omega)$ for $i=1, \ldots, d$, we get

$$
\lambda_{1, \beta}(\Omega) \leq \frac{d}{S(\Omega)} \leq d \frac{|\Omega|+\beta \Lambda[\Omega]}{\int_{\partial \Omega}|x|^{2}} \leq \frac{d}{1+\gamma_{d}\left(\frac{\left|\Omega \Delta B_{R}\right|}{\left|B_{R}\right|}\right)^{2}} \frac{|\Omega|+\beta \Lambda[\Omega]}{d \omega_{d}^{-1 / d}|\Omega|^{\frac{d+1}{d}}} .
$$

Proof of Theorem 1.3. It is a direct adaptation of the previous proof to the Laplace-Beltrami case: it suffices to replace the bilinear form $A_{\beta}(u, v)$ by $A(u, v)=\int_{\Omega} \nabla u$. $\nabla v$. Then Eq. (24) becomes $A\left(w_{i}, w_{i}\right)=\left(C P[\Omega] C^{T}\right)_{i i} \leq$ $\Lambda[\Omega]$ and the conclusion follows.

\subsection{On the sharpness of the upper bounds}

Testing the sharpness. Let us denote by $M_{1}(\Omega)$ the upper bound (11). In order to emphasize the improvement to the inequality of Brasco, De Philippis and Ruffini, we also plot the rougher upper bound

$$
M_{3}(\Omega)=\frac{|\Omega|+\beta \Lambda[\Omega]}{\omega_{d}^{-1 / d}|\Omega|^{\frac{d+1}{d}}}=d \frac{|\Omega|+\beta \Lambda[\Omega]}{R^{2}\left|\partial B_{R}\right|} .
$$

It is clear from the bound of $\Lambda[\Omega]$ stated in (22) that

$$
\lambda_{1, \beta}\left(B_{R}\right)=M_{1}\left(B_{R}\right) \leq M_{2}(\Omega)=\frac{d}{\left(1+\gamma_{d} \frac{\left|\Omega \Delta B_{R}\right|}{\left|B_{R}\right|}\right)^{2}} \frac{|\Omega|+\beta \Lambda[\Omega]}{R^{2}\left|\partial B_{R}\right|} .
$$

We also plot the shaper bound

$$
M_{1}(\Omega)=d \frac{|\Omega|+\beta \Lambda[\Omega]}{\int_{\partial \Omega}|x|^{2}} .
$$

This inequality means that proving that balls are maximizers would be strictly better than (11). Let us illustrate this fact with some numerical illustrations. We compute $\lambda_{1, \beta}(\Omega)$ and $M_{i}(\Omega)(i=1,2)$ for several parametrized families of plane domains when $\beta=1$. In Fig. 1(a), we present the case of ellipses of area $\pi$ (their semiaxis are $e^{t}$ and $e^{-t}, t$ is in abscissa) while in Fig. 1(b) and 1(c) we present the case of the star-shaped domains $\Omega_{t}$ defined in polar coordinate by $r(\theta)=a(t)(2+\cos (k \theta))$ where $a(t)$ is a constant chosen such that $\left|\Omega_{t}\right|=\pi$.

From these graphs, it seems that the upper bounds $M_{i}(\Omega)$ lack of precision when $\Omega$ is far from a ball and that the maximality of balls is possible and would improve the upper bound given in Corollary 1.2. 


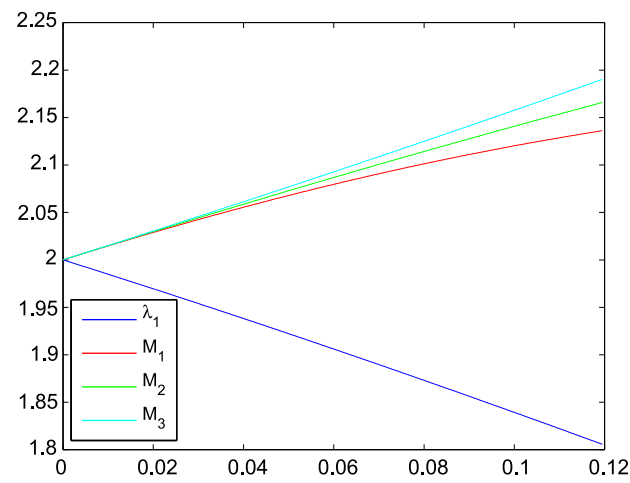

(a) Ellipses of area $\pi$,

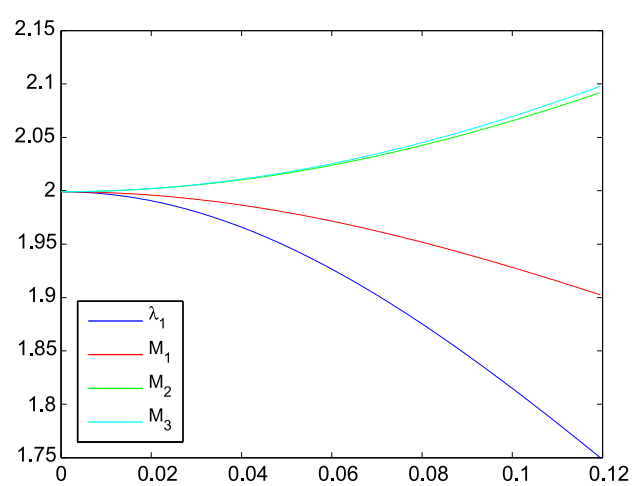

(c) 11 branches star-shaped domains

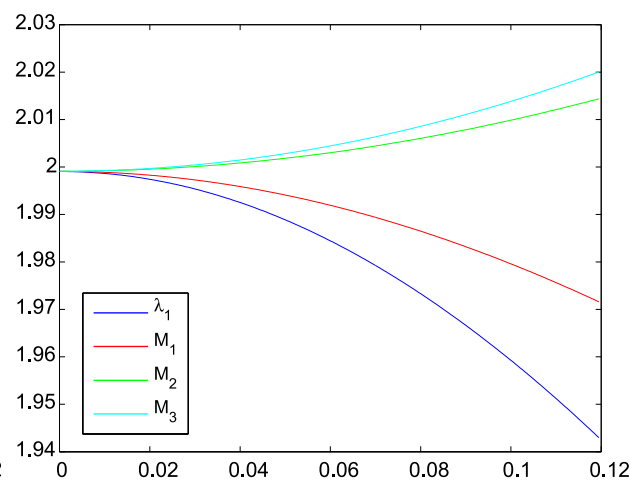

(b) 5 branches star-shaped domains

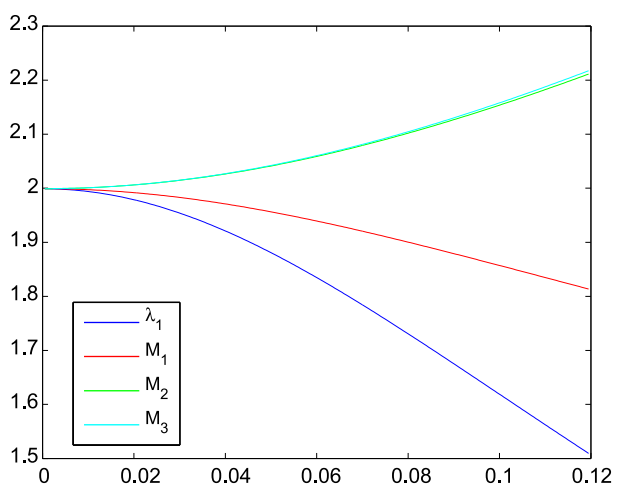

(d) 17 branches star-shaped domains

Fig. 1. Comparison of $\lambda_{1, \beta}(\Omega)$ and $M_{i}(\Omega)$. Here $\lambda_{1, \beta}\left(B_{1}\right)=2$. (For interpretation of the references to color in this figure legend, the reader is referred to the web version of this article.)

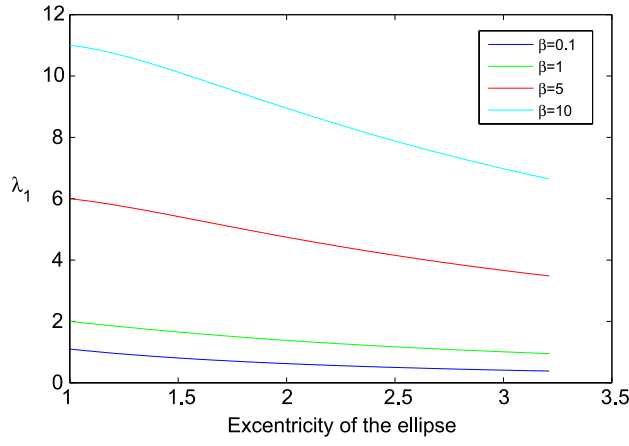

(a) $|\Omega|=\pi$

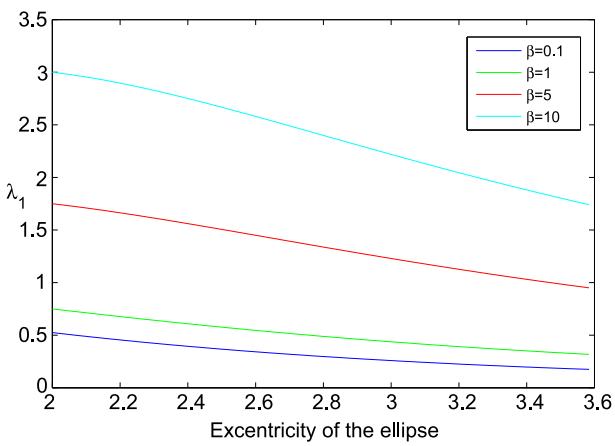

(b) $|\Omega|=4 \pi$

Fig. 2. $\lambda_{1, \beta}(\Omega)$ when $\Omega$ is an ellipse of volume $|\Omega|$. (For interpretation of the references to color in this figure legend, the reader is referred to the web version of this article.)

Some numerical tests. It is natural to wonder if the ball has the largest $\lambda_{1, \beta}$ among all the domains of the same volume that are homeomorphic to the ball. This question cannot be solved with estimate (11), as Fig. 1(a) shows. Therefore, to conclude this section, we would like to present some numerical experiments in favor of such property.

Let us start by computing the value of $\lambda_{1, \beta}(\Omega)$ when $\Omega$ is an ellipse of fixed volume (see Fig. 2). We present here the results of our numerical computations for $\beta \in\{0.1,1,5,10\}$ when $|\Omega|=\pi$, then when $|\Omega|=4 \pi$. In both figures, the abscissa stands for the eccentricity of the ellipse. It seems that the ball maximizes $\lambda_{1, \beta}$ among ellipses of fixed area. 


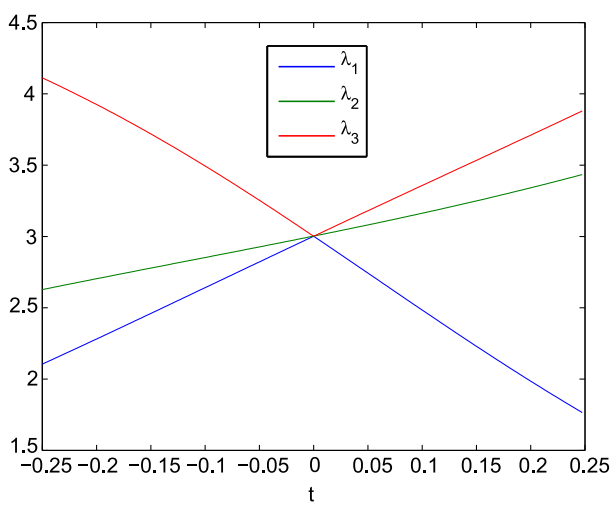

(a) $\alpha=(2,-0.8,-1.2)$

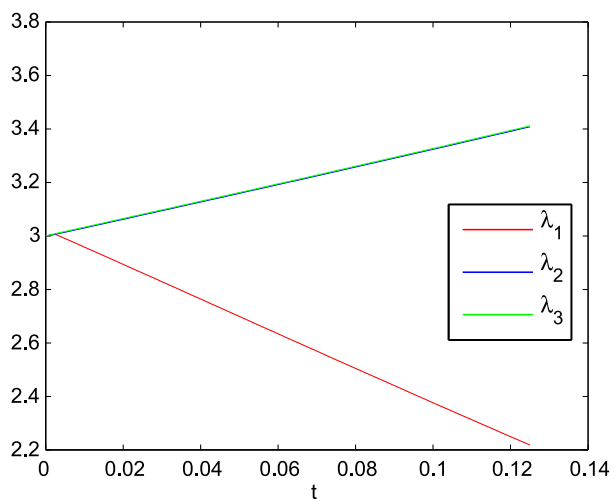

(b) $\alpha=(2,-1,-1)$

Fig. 3. $\left(\lambda_{1, \beta}\left(\Omega_{t}\right), \lambda_{2, \beta}\left(\Omega_{t}\right), \lambda_{3, \beta}\left(\Omega_{t}\right)\right)$ when $\Omega_{t}$ is a parametrized ellipsoid of volume $4 \pi / 3$. (For interpretation of the references to color in this figure legend, the reader is referred to the web version of this article.)

Let us show some computations in dimension three. We consider families of ellipsoids with semi-axes defined by $\left(\exp \left(\alpha_{i} t\right)\right)_{i=1,2,3}$ where $\alpha_{1}+\alpha_{2}+\alpha_{3}=0$ to insure the volume constraint. The ball $B$ corresponds to $t=0$. We remind that in this case, $\lambda_{1, \beta}(B)$ has multiplicity 3 at the sphere, we then have plotted the three corresponding eigenvalues in two cases: first for the family such that $\alpha=(2,-0.8,-1.2)$ in Fig. 3(a), then for $\alpha=(2,-1,-1)$ in Fig. 3(b). In the last case, the defined ellipsoids are of revolution and we observe that in this particular case $\lambda_{3, \beta} \approx \lambda_{4, \beta}$. One can wonder if it is really the case.

Let $E(a, b)$ be an ellipsoid of volume $4 \pi / 3$ where $a$ is the larger semiaxis and $b$ the middle one. We now show in Fig. 4 the surfaces $z=\lambda_{i, \beta}(E(a, b))$ where $i=1,2,3$. The pictures have been obtained by interpolation after the computations of the eigenvalues on 2700 ellipsoids. Again one can attest that the ball seems to maximize $\lambda_{1, \beta}$ among ellipsoids.

\section{First order shape calculus}

In order to go one step further, we adopt a shape optimization point of view and prove in this section that the ball is a critical point. The main difficulty here is that the eigenvalue $\lambda_{1, \beta}(B)$ has multiplicity the dimension of the ambient space. We need some technical material on shape derivative and tangential calculus on manifold to justify the results stated in this section; to simplify the reading of this work, we postpone these reminders in Appendix A.

Let us emphasize that from this point we do not make the assumption $\beta \geq 0$, and therefore all the results of this section and the following are valid for any $\beta \in \mathbb{R}$. Thus from now on we drop the notation $\beta$ in $\lambda_{1, \beta}$ since there is no possible confusion anymore.

\subsection{Notations and preliminary result for shape deformation}

We adopt the formalism of Hadamard's shape calculus and consider the map $t \mapsto T_{t}=I+t \boldsymbol{V}$ where $\boldsymbol{V} \in$ $W^{3, \infty}\left(\Omega, \mathbb{R}^{d}\right)$ and $t$ is small enough. We denote

$$
\Omega_{t}=T_{t}(\Omega)=\{x+t \boldsymbol{V}(x), x \in \Omega\} .
$$

Remark 3.1. More generally the results and computations from this section are valid if $t \mapsto T_{t}$ satisfies:

- $T_{0}=I d$,

- for every $t$ near $0, T_{t}$ is a $W^{3, \infty}$-diffeomorphism from $\Omega$ onto its image $\Omega_{t}=T_{t}(\Omega)$,

- the application $t \mapsto T_{t}$ is real-analytic near $t=0$.

We need to introduce the surface jacobian $\omega_{t}$ defined as

$$
\omega_{t}(x)=\operatorname{det}\left(D T_{t}(x)\right)\left\|\left(D T_{t}(x)^{T}\right)^{-1} \mathbf{n}(x)\right\|,
$$




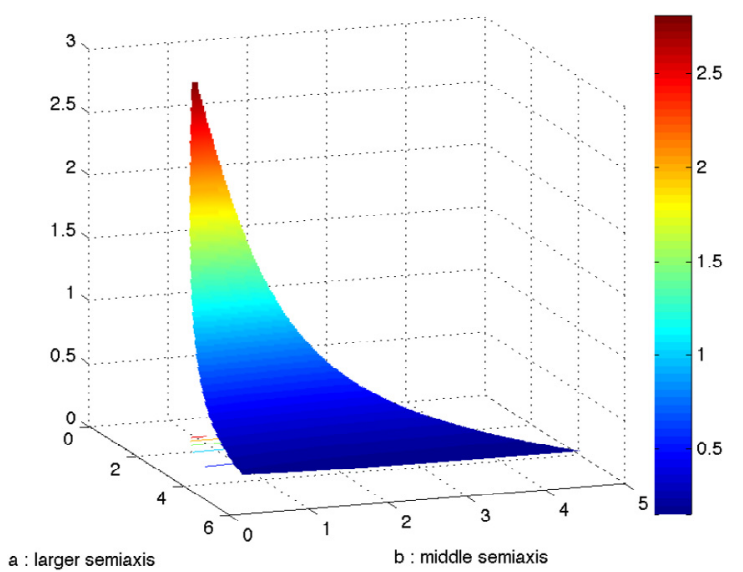

(a) $\lambda_{1, \beta}(E(a, b))$

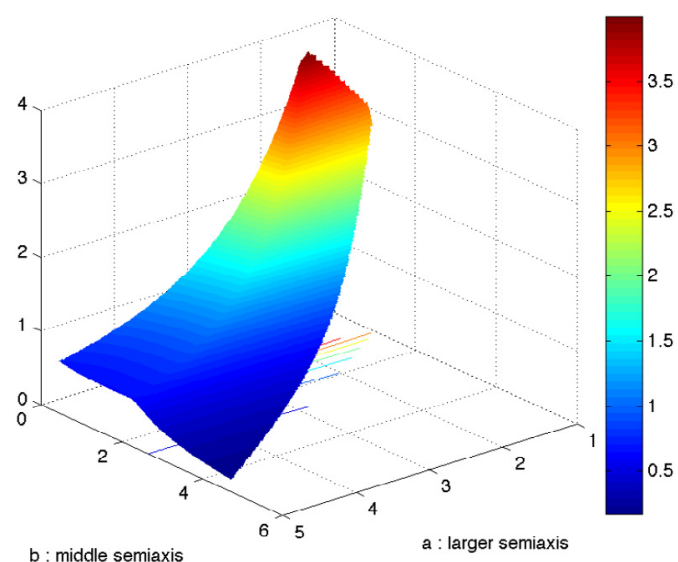

(b) $\lambda_{2, \beta}(E(a, b))$

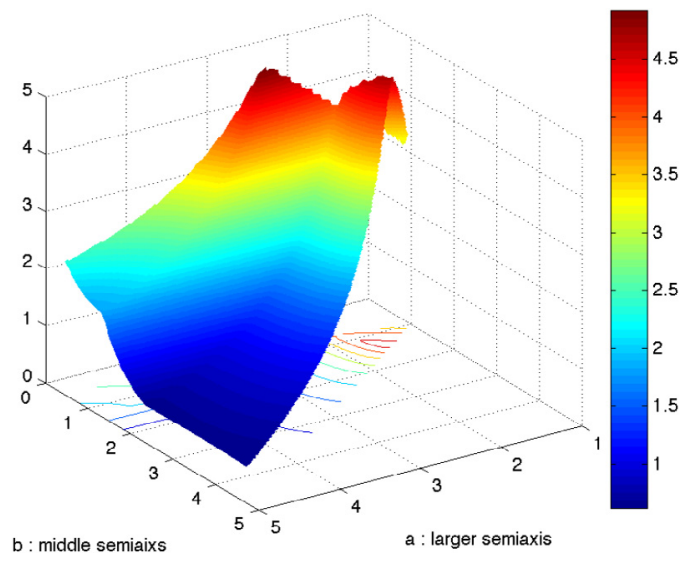

(c) $\lambda_{3, \beta}(E(a, b))$

Fig. 4. $\left(\lambda_{1, \beta}(\Omega), \lambda_{2, \beta}(\Omega), \lambda_{3, \beta}(\Omega)\right)$ when $\Omega=E(a, b)$ is an ellipsoid of volume $4 \pi / 3$. (For interpretation of the references to color in this figure legend, the reader is referred to the web version of this article.)

and the functions

$$
A_{t}(x)=\left(D T_{t}(x)\right)^{-1}\left(D T_{t}(x)^{T}\right)^{-1}, \quad \tilde{A}_{t}(x)=\operatorname{det}\left(D T_{t}(x)\right) A_{t}(x), \quad C_{t}(x)=\omega_{t}(x) A_{t}(x) .
$$

We have to study the transport of the considered eigenvalue problem on the deformed domain $\Omega_{t}$. To that end, we first rewrite the deformed equation on the fixed domain $\Omega$ and its boundary $\partial \Omega$ : we have to describe how are transported the Laplace-Beltrami and the Dirichlet-to-Neumann operators.

Transport of the Dirichlet-to-Neumann map. Let us consider the Dirichlet-to-Neumann operator defined on its natural space $\mathrm{D}_{t}: H^{1 / 2}\left(\partial \Omega_{t}\right) \rightarrow H^{-1 / 2}\left(\partial \Omega_{t}\right)$. It maps a function $\phi_{t}$ in $H^{1 / 2}\left(\partial \Omega_{t}\right)$ onto the normal derivative of its harmonic expansion in $\Omega_{t}$, that is to say $\mathrm{D}_{t}\left(\phi_{t}\right)=\partial_{\boldsymbol{n}_{t}} u_{t}$, where $u^{t}$ solves the boundary values problem:

$$
\begin{cases}-\Delta u_{t}=0 & \text { in } \Omega_{t} \\ u_{t}=\phi_{t} & \text { on } \partial \Omega_{t}\end{cases}
$$

To compute the quantity $\mathcal{D}_{t}$ such that $\mathcal{D}_{t}\left(\phi_{t} \circ T_{t}\right)=\left[\mathrm{D}_{t}\left(\phi_{t}\right)\right] \circ T_{t}$, we transport the boundary value problem (25) back on the domain $\Omega$. In others words, $\mathcal{D}_{t}$ makes the following diagram commutative: 


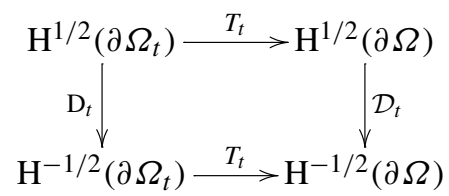

To be more precise, we have the following result proved in [12].

Lemma 3.2. Given $\psi \in H^{1 / 2}(\partial \Omega)$, we denote by $v^{t}$ the solution of the boundary value problem

$$
\begin{cases}-\operatorname{div}\left(\tilde{A}_{t} \nabla v^{t}\right)=0 & \text { in } \Omega \\ v^{t}=\psi & \text { on } \partial \Omega\end{cases}
$$

and then define $\mathcal{D}_{t} \psi \in H^{-1 / 2}(\partial \Omega)$ as:

$$
\mathcal{D}_{t} \psi: f \in H^{1 / 2}(\partial \Omega) \mapsto \int_{\Omega} \tilde{A}_{t}(x) \nabla v^{t}(x) \cdot \nabla E(f)(x) d x,
$$

where $E$ is a continuous extension operator from $H^{1 / 2}(\partial \Omega)$ to $H^{1}(\Omega)$. Then the relation

$$
\left(\mathrm{D}_{t} \varphi\right) \circ T_{t}=\mathcal{D}_{t}\left[\varphi \circ T_{t}\right]
$$

holds for all functions $\varphi \in H^{1 / 2}\left(\Omega_{t}\right)$.

Setting $u^{t}=u_{t} \circ T_{t}$, we check from the variational formulation, that the function $u^{t}$ is the unique solution of the transported boundary value problem:

$$
\begin{cases}-\operatorname{div}\left(\tilde{A}_{t} \nabla u^{t}\right)=0 & \text { in } \Omega \\ u^{t}=\phi_{t} \circ T_{t} & \text { on } \partial \Omega .\end{cases}
$$

Hence, setting $y=T_{t}(x), x \in \Omega$ we get formally

$$
\mathrm{D}_{t}\left(\phi_{t}\right)(y)=\nabla u_{t}(y) \cdot \boldsymbol{n}_{t}(y)=\left(D T_{t}(x)^{T}\right)^{-1} \nabla v_{t}(x) \cdot \frac{\left(D T_{t}(x)^{T}\right)^{-1} \boldsymbol{n}(x)}{\left\|\left(D T_{t}(x)^{T}\right)^{-1} \boldsymbol{n}(x)\right\|}=\frac{A_{t}(x) \boldsymbol{n}(x) \cdot \nabla u^{t}(x)}{\left\|\left(D T_{t}(x)^{T}\right)^{-1} \boldsymbol{n}(x)\right\|} .
$$

Here again, we can give a sense to the co-normal derivative $A_{t} \boldsymbol{n} . \nabla u^{t}$ thanks to the boundary value problem (28): this quantity is defined in a weak sense as the previous Dirichlet-to-Neumann operator $\mathrm{D}_{t}$.

Transport of the Laplace-Beltrami operator. We recall now the expression of the transported Laplace-Beltrami operator, relying on the relation

$$
\forall \varphi \in H^{2}\left(\partial \Omega_{t}\right), \quad\left(\Delta_{\tau} \varphi\right) \circ T_{t}=\frac{1}{\omega_{t}(x)} \operatorname{div}_{\tau}\left(C_{t}(x) \nabla_{\tau}\left(\varphi \circ T_{t}\right)(x)\right) \quad \text { on } \partial \Omega .
$$

Let us denote by $\mathcal{L}_{t}$ the operator defined as

$$
\mathcal{L}_{t}\left[\varphi \circ T_{t}\right](x)=\frac{1}{\omega_{t}(x)} \operatorname{div}_{\tau}\left\{C_{t}(x) \nabla\left[\varphi \circ T_{t}\right](x)-\frac{C_{t}(x) \nabla\left[\varphi \circ T_{t}\right](x) . \boldsymbol{n}(x)}{A_{t}(x) \boldsymbol{n}(x) . \boldsymbol{n}(x)} A_{t}(x) \boldsymbol{n}(x)\right\}
$$

for $\varphi \in H^{5 / 2}\left(\Omega_{t}\right)$. In [12], we show the following lemma:

\section{Lemma 3.3. The identity}

$$
\left[\Delta_{\tau} \varphi\right] \circ T_{t}=\mathcal{L}_{t}\left[\varphi \circ T_{t}\right]
$$

holds for all functions $\varphi$ belonging to $H^{5 / 2}\left(\Omega_{t}\right)$. 


\subsection{Regularity of the eigenfunctions and eigenvalues with respect to the parameter}

The section is a slight variation of a theorem due to Ortega and Zuazua on the existence and regularity of eigenvalues and associated eigenfunctions in the case of Stokes system [24]. The difficulty comes from the possible multiple eigenvalues. The main result is, for a fixed deformation field $\boldsymbol{V} \in W^{3, \infty}\left(\Omega, \mathbb{R}^{d}\right)$, the existence of smooth branches of eigenvalue. In other words, the eigenvalues are not regular when sorted in the increasing order, but they can be locally relabeled around the multiple point in order to remain smooth. The restriction is that this labeling depends on the deformation field $\boldsymbol{V}$ hence one cannot hope to prove Fréchet-differentiability.

Theorem 3.4. Let $\Omega$ be an open smooth bounded domain of $\mathbb{R}^{d}$. Assume that $\lambda$ is an eigenvalue of multiplicity $m$ of the Wentzell-Laplace operator. We suppose that $T_{t}=I+t \boldsymbol{V}$ for some $\boldsymbol{V} \in W^{3, \infty}(\Omega, \mathbb{R})^{d}$ and denote $\Omega_{t}=T_{t}(\Omega)$. Then there exist $m$ real-valued continuous functions $t \mapsto \lambda_{i}(t), i=1,2, \ldots, m$, and $m$ functions $t \mapsto u_{i}^{t} \in H^{\frac{5}{2}}(\Omega)$ such that the following properties hold

1. $\lambda_{i}(0)=\lambda, i=1, \ldots, m$.

2. The functions $t \mapsto \lambda_{i}(t)$ and $t \mapsto u_{i}^{t}, i=1,2, \ldots, m$, are analytic in a neighborhood of $t=0$.

3. The functions $u_{i, t}$ defined by $u_{i, t} \circ T_{t}=u_{i}^{t}$ are normalized eigenfunctions associated to $\lambda_{i}(t)$ on the moving domain $\Omega_{t}$. If one considers $K$ compact subset such that $K \subset \Omega_{t}$ for all $t$ small enough, then $t \mapsto u_{i, t \mid K}$ is also an analytic function of $t$ in a neighborhood of $t=0$.

4. Let $I \subset \mathbb{R}$ be an interval such that $\bar{I}$ contains only the eigenvalue $\lambda$ of the Wentzell problem of multiplicity $m$. Then there exists a neighborhood of $t=0$ such that $\lambda_{i}(t), i=1, \ldots, m$, are the only eigenvalues of $\Omega_{t}$ which belongs to $I$.

Proof. Let $\lambda$ be an eigenvalue of multiplicity $m$ and let $u_{1}, \ldots, u_{m}$ be the orthonormal eigenfunctions associated to $\lambda$. Let $\left(\lambda(t), u_{t}\right)$ be an eigenpair satisfying

$$
\left(P_{t}\right) \begin{cases}-\Delta u_{t}=0 & \text { in } \Omega_{t}, \\ -\beta \Delta_{\tau} u_{t}+\partial_{n_{t}} u_{t}=\lambda(t) u_{t} & \text { on } \partial \Omega_{t} .\end{cases}
$$

Setting $u^{t}=u_{t} \circ T_{t}$, Lemmas 3.2 (transport of the Dirichlet-to-Neumann map) and 3.3 (transport of the LaplaceBeltrami operator) show that the system $\left(P_{t}\right)$ above is equivalent to the following equation set on the boundary

$$
\left(-\beta \mathcal{L}_{t}+\mathcal{D}_{t}\right) u^{t}=\lambda(t) \omega_{t} u^{t} \quad \text { on } \partial \Omega .
$$

Consider the operator $S(t)$ defined on $\mathrm{H}^{3 / 2}(\partial \Omega)$ by

$$
v \mapsto S(t) v=-\beta \mathcal{L}_{t} v+\mathcal{D}_{t} v
$$

From their expressions computed for example in [17, Section 5-2] and the regularity assumption on $T_{t}$, all the operators $C_{t}, A_{t}$ and $\omega_{t}$ are analytic in a neighborhood of $t=0$. Since $\operatorname{det}\left(D T_{t}\right)>0$ for $t$ small enough, we deduce that all the expressions involved in $\mathcal{C}_{t}, \mathcal{L}_{t}$ and $\mathcal{D}_{t}$ are analytic in a neighborhood of $t=0$. This enables us to conclude that $S(t)$ is also analytic in a neighborhood of zero.

To show that the eigenvalues and the corresponding eigenfunctions are analytic in a neighborhood of zero, we apply the Lyapunov-Schmidt reduction in order to treat a problem on a finite dimensional space, namely the kernel of $S(0)-\lambda I$. To that end, we rewrite the problem $\left(P_{t}\right)$ on the fixed domain $\partial \Omega$ as

$$
S(t)\left(u^{t}\right)-\lambda(t) \omega_{t} u^{t}=0 .
$$

From the decomposition

$$
(S(0)-\lambda)\left(u^{t}\right)=\left[(S(0)-S(t))+\left[(\lambda(t)-\lambda) \omega_{t}+\lambda\left(\omega_{t}-1\right)\right]\right] u^{t},
$$

$u^{t}$ is solution of the equation

$$
(S(0)-\lambda)\left(u^{t}\right)=W(t, \lambda(t)-\lambda) u^{t},
$$

where we have set $R(t)=S(0)-S(t)+\lambda\left(\omega_{t}-1\right)$ and $W(t, \alpha)=R(t)+\alpha \omega_{t} I$. From the Lyapunov-Schmidt Theorem (see [24, Lemma 3-2, p. 999]), we obtain that $S(0)-\lambda$ has a right inverse operator denoted by $K$. Hence the equation 
above implies that $u^{t}=K W(t, \lambda(t)-\lambda) u^{t}+\psi_{t}$ where $\psi_{t} \in \operatorname{Ker}(S(0)-\lambda)$, i.e. $\psi_{t}=\sum_{k=1}^{m} c_{k}(t) \phi_{k}$ where $\left(\phi_{k}\right)$ is a basis of $\operatorname{Ker}(S(0)-\lambda)$. Notice that $I-K W(t, \lambda(t)-\lambda)$ is invertible on $\operatorname{Ker}(S(0)-\lambda I)$, the inverse of his operator restricted to this kernel will be denoted by $(I-K W(t, \lambda(t)-\lambda))^{-1}$ so that

$$
u^{t}=(I-K W(t, \lambda(t)-\lambda))^{-1} \psi_{t} .
$$

From (34), $W(t, \lambda(t)-\lambda) u^{t}$ belongs to $\operatorname{Im}(S(0)-\lambda)=\operatorname{Ker}^{\perp}(S(0)-\lambda)$ since $S(0)$ is a Fredholm selfadjoint operator, and then

$$
\sum_{k=1}^{m} c_{k}(t)\left\langle W(t, \lambda(t)-\lambda)(I-K W(t, \lambda(t)-\lambda))^{-1} \phi_{k}, \phi_{i}\right\rangle=0, \quad i=1,2, \ldots, m,
$$

where $\langle\cdot, \cdot\rangle$ denotes the scalar product of $L^{2}(\partial \Omega)$. This shows that a vector of coefficients $C=\left(c_{j}\right)_{j=1, \ldots, m} \neq 0$ is a solution if and only if the determinant of the $m \times m$ matrix $M(t, \lambda(t)-\lambda)$ with entries

$$
M(t, \alpha)_{i, j}=\left\langle W(t, \alpha)(I-K W(t, \alpha))^{-1} \phi_{j}, \phi_{i}\right\rangle
$$

satisfies

$$
\operatorname{det}(M(t, \lambda(t)-\lambda))=0 .
$$

Hence $\lambda(t)$ is an eigenvalue of our problem if and only if $\operatorname{det}(M(t, \lambda(t)-\lambda))=0$. Note that $t \mapsto M(t, \lambda(t))$ is analytic around $t=0$.

For small values of $t$ the operator $(I-K W(t, \alpha))^{-1}$ is well defined since $I-K W(0,0)=I$ and $t \mapsto(I-$ $K W(t, \alpha))^{-1}$ is analytic around $t=0$. On the other hand, if $\operatorname{det} M(t, \alpha)=0$ then (35) has a nontrivial solution $c_{1}(t), \ldots, c_{m}(t)$ and this means that $\lambda(t)=\lambda+\alpha$ is an eigenvalue of $\left(P_{t}\right)$.

We focus now on $\operatorname{det} M(t, \alpha)$ for $\alpha \in \mathbb{R}$. From the fact that $W(0, \alpha)=\alpha I$, it comes that for sufficiently small values of $\alpha$, the operator $I-K W(0, \alpha)$ is invertible on $\operatorname{Ker}(S(0)-\lambda I)$ and from the Von Neumann expansion we write

$$
\left\langle W(0, \alpha)(I-K W(0, \alpha))^{-1} \phi_{i}, \phi_{j}\right\rangle=\alpha\left[\delta_{i j}+\sum_{k=1}^{\infty} \alpha^{k}\left\langle K^{k} \phi_{i}, \phi_{j}\right\rangle\right]
$$

hence

$$
\operatorname{det}(M(0, \alpha))=\alpha^{m}+\sum_{i=1}^{\infty} \beta_{i} \alpha^{m+i}=\alpha^{m}\left(1+\sum_{i=1}^{\infty} \beta_{i} \alpha^{i}\right) .
$$

Since $\operatorname{det}(M(0, \alpha)) \neq 0$ is the restriction on $t=0$ of $\operatorname{det}(M(t, \alpha))$, we deduce from the Weierstrass preparation theorem that there is neighborhood of $(0,0)$ such that $\operatorname{det}(M(t, \alpha))$ is uniquely representable as

$$
\operatorname{det}(M(t, \alpha))=P_{m}(t, \alpha) h(t, \alpha)
$$

where

$$
P_{m}(t, \alpha)=\alpha^{m}+\sum_{k=1}^{m} a_{k}(t) \alpha^{m-k}
$$

and where

$$
h(t, \alpha) \neq 0 .
$$

Furthermore, the coefficients $a_{k}(t), k=1, \ldots, m$, are real and analytic in a neighborhood of $t=0$. Then $\operatorname{det}(M(t, \alpha))=0$ if and only if $P_{m}(t, \alpha)=0$. If $\alpha_{k}(t), k=1, \ldots, m$, are the real roots of the polynomial, we take $\lambda_{1}(t)=\lambda+\alpha_{1}(t)$ if $\alpha_{1}(t)$ is not identically equal to zero.

We now have to find the $(m-1)$ other branches $\lambda_{i}(t)$ and the corresponding eigenfunction $u_{i, t}$ for $i=2, \ldots, m$. We use the idea of the deflation method by considering the operator

$$
S_{2}(t)=S(t)-\lambda_{1} P_{1}(t)
$$

where $P_{1}$ is the orthogonal projection on the subspace spanned by $u_{1, t}$. At $t=0$, we obtain

$$
S_{2}(0) u_{j}=S(0) u_{j}-\lambda \delta_{1 j} u_{j}
$$


in other terms $S_{2}(0) u_{j}=\lambda u_{j}, j=2, \ldots, m$, while $S_{2}(0) u_{1}=0$. This shows that $\lambda$ is an eigenvalue of multiplicity $m-1$ of $S_{2}(0)$ with eigenvalues $u_{2}, \ldots, u_{m}$. One can show that these functions are the only linearly independent eigenfunctions associated to $\lambda$. Now we can apply the same recipe used before to the operator $S_{2}$ instead of $S$. We then get a branch $\lambda_{2}(t)$ such that $t \mapsto \lambda_{2}(t)$ is analytic in a neighborhood of $t=0$. Iterating the process, we get at the end the $m$ branches $\lambda_{i}(t), i=1, \ldots, m$, such that each branch is analytic in a neighborhood of $t=0$ and $m$ corresponding eigenfunctions forming an orthonormal set of functions in $H^{\frac{3}{2}}\left(\partial \Omega_{t}\right)$.

The proof of the last item follows the same lines as the proof of Ortega and Zuazua for the Stokes system, see [24].

Theorem 3.5. With the notations of Theorem 3.4, if $t \mapsto\left(\lambda(t), u_{t}\right)$ is one of the smooth eigenpair path $\left(\lambda_{i}(t), u_{i, t}\right)$ of $\Omega_{t}$ for the Wentzell problem, then the shape derivative $u^{\prime}=\left(\partial_{t} u_{t}\right)_{\mid t=0}$ of the eigenfunction satisfies

$$
\begin{aligned}
& \Delta u^{\prime}=0 \quad \text { in } \Omega, \\
&-\beta \Delta_{\tau} u^{\prime}+\partial_{n} u^{\prime}-\lambda u^{\prime}= \beta \Delta_{\tau}\left(V_{n} \partial_{n} u\right)-\beta \operatorname{div}_{\tau}\left(V_{n}\left(2 D^{2} b-H I_{d}\right) \nabla_{\tau} u\right) \\
&+\operatorname{div}_{\tau}\left(V_{n} \nabla_{\tau} u\right)-\lambda^{\prime}(0) u+\lambda V_{n}\left(\partial_{n} u+H u\right) \quad \text { on } \partial \Omega .
\end{aligned}
$$

Proof. The fact that $u^{\prime}$ is harmonic inside the domain is trivial. To derive the boundary condition satisfied by $u^{\prime}$, we use a test function $\phi_{t}$ defined on $\partial \Omega_{t}$ with $\partial_{n} \phi_{t}=0$ as used in the proof of Lemmas 3.2 and 3.3 in [12]. We get the following weak formulation valid for all $t$ small enough:

$$
\int_{\partial \Omega_{t}} \beta \nabla_{\tau} u(t, x) \cdot \nabla_{\tau} \phi_{t} d \sigma_{t}+\int_{\partial \Omega_{t}} \partial_{n_{t}} u(t, x) \phi_{t} d \sigma_{t}-\lambda(t) \int_{\partial \Omega_{t}} u(t, x) \phi_{t} d \sigma_{t}=0 .
$$

We take the derivative with respect to $t$ and get at $t=0$ :

$$
\left.\beta \frac{d}{d t}\left(\int_{\partial \Omega_{t}} \nabla_{\tau} u(t, x) \cdot \nabla_{\tau} \phi_{t} d \sigma_{t}\right)\right|_{t=0}+\left.\frac{d}{d t}\left(\int_{\partial \Omega_{t}} \partial_{n_{t}} u(t, x) \phi_{t} d \sigma_{t}\right)\right|_{t=0}=\left.\frac{d}{d t}\left(\lambda(t) \int_{\partial \Omega_{t}} u(t, x) \phi_{t}(x) d \sigma_{t}\right)\right|_{t=0} .
$$

From [14] and [7], we get

$$
\left.\frac{d}{d t}\left(\int_{\partial \Omega_{t}} \nabla_{\tau} u(t, x) \cdot \nabla_{\tau} \phi_{t} d \sigma_{t}\right)\right|_{t=0}=\int_{\partial \Omega}\left(-\Delta_{\tau} u^{\prime}-\Delta_{\tau}\left(V_{n} \partial_{n} u\right)+\operatorname{div}_{\tau}\left(\left(2 D^{2} b-H I_{d}\right) \nabla_{\tau} u\right)\right) \phi d \sigma .
$$

After some lengthy but straightforward computations we also obtain

$$
\left.\frac{d}{d t}\left(\int_{\Omega_{t}} \partial_{n_{t}} u \phi_{t} d \sigma_{t}\right)\right|_{t=0}=\int_{\partial \Omega} \partial_{n} u^{\prime} \phi d \sigma-\int_{\partial \Omega} \nabla_{\tau} V_{n} \cdot \nabla_{\tau} u \phi d \sigma+\int_{\partial \Omega} V_{n}\left(\partial_{n} u+H u\right) \phi d \sigma
$$

and

$$
\left.\frac{d}{d t}\left(\int_{\partial \Omega_{t}} \lambda(t) u_{t} \phi_{t} d \sigma_{t}\right)\right|_{t=0}=\lambda^{\prime}(0) \int_{\partial \Omega} u \phi d \sigma+\lambda \int_{\partial \Omega} u^{\prime} \phi d \sigma+\lambda \int_{\partial \Omega} \partial_{n} u \phi d \sigma+\lambda \int_{\partial \Omega} H u \phi d \sigma .
$$

To end the proof of this second point, it suffices to gather the relations.

\subsection{Shape derivative of simple eigenvalues of the Wentzell-Laplace problem}

Let $\lambda$ be a simple eigenvalue of the Wentzell-Laplace equation (1) and let $u$ be the corresponding normalized eigenfunction. We give in this subsection the explicit formula for the shape derivative of the eigenvalue of the Wentzell-Laplace operator associated to (1).

On $\Omega_{t}=(I+t \boldsymbol{V})(\Omega)$ with $t$ small, there is a unique eigenvalue $\lambda(t)$ near $\lambda$ which is an analytic function with respect of the parameter $t$. The associated eigenfunction $u_{t}(x)=u(t, x)$ is solution of the problem (1). The shape derivative denoted by $u^{\prime}$ is the partial derivative $\partial_{t} u(t, x)$ evaluated at $t=0$ and solves (36). Let us deduce the analytic expression of $\lambda^{\prime}(0)$ : 
Theorem 3.6. If $(\lambda, u)$ is an eigenpair (with $u$ normalized) for the Wentzell problem with the additional assumption that $\lambda$ is simple then the application $t \rightarrow \lambda(t)$ is analytic and its derivative at $t=0$ is

$$
\lambda^{\prime}(0)=\int_{\partial \Omega} V_{n}\left(\left|\nabla_{\tau} u\right|^{2}-\left|\partial_{n} u\right|^{2}-\lambda H|u|^{2}+\beta\left(H I_{d}-2 D^{2} b\right) \nabla_{\tau} u . \nabla_{\tau} u\right) d \sigma .
$$

Proof. We start with the result of Theorem 3.5. Let us multiply the two sides of (36) the boundary condition satisfied by $u^{\prime}$ by the eigenfunction $u$ and integrate over the boundary $\partial \Omega$ :

$$
\begin{aligned}
0= & \int_{\partial \Omega} v^{\prime}\left(-\beta \Delta_{\tau} u+\partial_{n} u-\lambda u\right) d \sigma+\int_{\partial \Omega} V_{n} \partial_{n} u\left(-\beta \Delta_{\tau} u\right) d \sigma \\
& +\int_{\partial \Omega} \beta V_{n}\left(H I_{d}-2 D^{2} b\right) \nabla_{\tau} u . \nabla_{\tau} u d \sigma+\int_{\partial \Omega} V_{n}\left|\nabla_{\tau} u\right|^{2}-\lambda^{\prime}(0) \int_{\partial \Omega}|u|^{2}-\lambda \int_{\partial \Omega} V_{n}\left(u \partial_{n} u+H|u|^{2}\right) d \sigma .
\end{aligned}
$$

Using the boundary condition satisfied by the eigenfunction: $-\beta \Delta_{\tau} u+\partial_{n} u-\lambda u=0$, it follows that

$$
\begin{aligned}
0= & \int_{\partial \Omega} V_{n} \partial_{n} u\left(\lambda u-\partial_{n} u\right) d \sigma+\int_{\partial \Omega} \beta V_{n}\left(H I_{d}-2 D^{2} b\right) \nabla_{\tau} u . \nabla_{\tau} u d \sigma \\
& +\int_{\partial \Omega} V_{n} \nabla_{\tau}|u|^{2}-\lambda^{\prime}(0) \int_{\partial \Omega}|u|^{2}-\lambda \int_{\partial \Omega} V_{n}\left(u \partial_{n} u+H|u|^{2}\right) d \sigma
\end{aligned}
$$

and the normalization condition $\int_{\partial \Omega} u^{2} d \sigma=1$ implies

$$
\lambda^{\prime}(0)=-\int_{\partial \Omega} V_{n}\left|\partial_{n} u\right|^{2} d \sigma+\int_{\partial \Omega} \beta V_{n}\left(H I_{d}-2 D^{2} b\right) \nabla_{\tau} u . \nabla_{\tau} u d \sigma+\int_{\partial \Omega} V_{n}\left|\nabla_{\tau} u\right|^{2}-\lambda \int_{\partial \Omega} V_{n} H|u|^{2} d \sigma .
$$

\subsection{Shape derivative of multiple eigenvalues of the Wentzell-Laplace problem}

\subsubsection{The general result}

We suppose that $\lambda$ is an eigenvalue of multiplicity $m$. For smooth deformation $t \mapsto \Omega_{t}$, there will be $m$ eigenvalues close to $\lambda$ (counting their multiplicities) for small values of $t$. We know that such a multiple eigenvalue is no longer differentiable in the classical sense. We are then led to compute the directional derivative of $t \mapsto \lambda_{i}(t)$ at $t=0$ where $\lambda_{i}(t), j=1, \ldots, m$, are given by Theorem 3.4. This is the second part of Theorem 1.4 that we recall here:

Theorem 3.7. Let $\lambda$ be a multiple eigenvalue of order $m \geq 2$. Then each $t \mapsto \lambda_{i}(t)$ for $i \in \llbracket 1, d \rrbracket$ given by Theorem 3.4 has a derivative near 0 , and the values of $\left(\lambda_{i}^{\prime}(0)\right)_{i \in \llbracket 1, d \rrbracket}$ are the eigenvalues of the matrix $M\left(V_{n}\right)=\left(M_{j k}\right)_{1 \leq j, k \leq m}$ defined by

$$
M_{j k}=\int_{\partial \Omega} V_{n}\left(\nabla_{\tau} u_{j} \cdot \nabla_{\tau} u_{k}-\partial_{n} u_{j} \partial_{n} u_{k}-\lambda H u_{j} u_{k}+\beta\left(H I_{d}-2 D^{2} b\right) \nabla_{\tau} u_{j} \cdot \nabla_{\tau} u_{k}\right) d \sigma
$$

Proof. Let $t \mapsto\left(u(t, x), \lambda(t)=\lambda\left(\Omega_{t}\right)\right)$ be a smooth path of eigenpair of the Laplace-Wentzell problem, so that it satisfies

$$
\begin{cases}\Delta u(t, x)=0 & \text { in } \Omega_{t} \\ -\beta \Delta_{\tau} u(t, x)+\partial_{n} u(t, x)=\lambda(t) u(t, x) & \text { on } \partial \Omega_{t} .\end{cases}
$$

We have proved that $u^{\prime}=\partial_{t} u(0, x)$ is harmonic in $\Omega$ and satisfies the boundary condition (36) on $\partial \Omega$. We use the decomposition of $u=u(0, x)$ as

$$
u=\sum_{j=1}^{m} c_{j} u_{j}
$$


for some $c=\left(c_{1}, c_{2}, \ldots, c_{m}\right)^{T} \neq 0$. Multiplying the two sides of Eq. (36) by $u_{k}$, we get after some integration by parts the eigenvalue equation

$$
\lambda^{\prime}(0) c=M c
$$

where $M=\left(M_{j k}\right)_{1 \leq i, j \leq m}$ is defined by (37). From this, we deduce that the set of derivatives $\left(\lambda_{i}^{\prime}(0)\right)_{i \in \llbracket 1, d \rrbracket}$ is exactly the set of eigenvalues of the matrix $M$, which achieves the proof of Theorem 3.7.

\subsubsection{The case of balls}

We consider now the case where the domain is a ball of radius $R$. The problem is invariant under translation. In order to remove the invariance, we fix the center of mass of the boundary of the domain, as in Section 2.

The coordinate functions $x_{i}$ are eigenfunctions of the Wentzell-Laplace operator, so we get

$$
\lambda=\frac{\beta(d-1)+R}{R^{2}}, \quad \text { and } \quad u_{i}(x)=\frac{x_{i}}{\left\|x_{i}\right\|_{L^{2}\left(\partial B_{R}\right)}}=\frac{x_{i}}{\sqrt{\omega_{d} R^{d+1}}} .
$$

Corollary 3.8. Let $\Omega=B_{R}$ be a ball of radius $R, \lambda_{1}$ its first non-trivial eigenvalue, which is of multiplicity $d$. The shape derivatives of the maps $t \mapsto \lambda_{i}(t), i=1, \ldots, d$, given by Theorem 3.4 are the eigenvalues of the matrix $M_{B_{R}}\left(V_{n}\right)=\left(M_{j k}\right)_{j, k=1, \ldots, d}$ defined by

$$
M_{j k}=\frac{\delta_{j k}}{\omega_{d} R^{d+1}}\left(1+\beta \frac{d-3}{R}\right) \int_{\partial B_{R}} V_{n}-C(d, R) \int_{\partial B_{R}} V_{n} x_{j} x_{k} d \sigma
$$

where $C(d, R)=\frac{(d+1)\left(1+\beta \frac{d-2}{R}\right)}{\omega_{d} R^{d+3}}$.

Proof. We use (37). On one hand we check the geometric quantities:

$$
H=\frac{d-1}{R}, \quad D^{2} b(x)=\frac{1}{R} I_{d}-\frac{1}{R^{3}}\left(x_{i} x_{j}\right)_{i, j}
$$

so since $\nabla_{\tau} u_{j}, \nabla_{\tau} u_{k}$ are in the tangent space of $\partial B_{R}$, we obtain that

$$
\left(H I_{d}-2 D^{2} b(x)\right) \nabla_{\tau} u_{j} \cdot \nabla_{\tau} u_{k}=\frac{d-3}{R} \nabla_{\tau} u_{j} \cdot \nabla_{\tau} u_{k}
$$

and on the other hand:

$$
\partial_{n} u_{j}=\frac{x_{i}}{R \sqrt{\omega_{d} R^{d+1}}} \quad \nabla_{\tau} u_{j} . \nabla_{\tau} u_{k}=\frac{1}{\omega_{d} R^{1+d}}\left(\delta_{j k}-\frac{x_{j} x_{k}}{R^{2}}\right)
$$

Therefore, the matrix $M=M_{B_{R}}$ has the following entries

$$
\begin{aligned}
M_{j k} & =\frac{1}{\omega_{d} R^{d+1}} \int_{\partial B_{R}} V_{n}\left[\left(\delta_{j k}-\frac{x_{j} x_{k}}{R^{2}}\right)-\frac{x_{j} x_{k}}{R^{2}}-\lambda \frac{d-1}{R} x_{j} x_{k}+\beta \frac{d-3}{R}\left(\delta_{j k}-\frac{x_{j} x_{k}}{R^{2}}\right)\right] d \sigma \\
& =\frac{\delta_{j k}}{\omega_{d} R^{d+1}}\left(1+\beta \frac{d-3}{R}\right) \int_{\partial B_{R}} V_{n}-\left[\frac{d+1+\beta \frac{(d-1)^{2}+d-3}{R}}{\omega_{d} R^{d+3}}\right] \int_{\partial B_{R}} V_{n} x_{j} x_{k} d \sigma .
\end{aligned}
$$

This leads to the result since $(d-1)^{2}+d-3=(d+1)(d-2)$.

From this formula, we deduce a first interesting result:

Proposition 3.9. If $V$ is a volume preserving deformation, then the following statements are equivalent:

(i) $V_{n}$ is orthogonal (in $\mathrm{L}^{2}\left(\partial B_{R}\right)$ ) to homogeneous harmonic polynomials of degree 2 ,

(ii) $M_{B_{R}}\left(V_{n}\right)=0$. 
Proof. We denote by $\mathcal{H}_{2}$ the space of homogeneous harmonic polynomials of degree 2 (therefore we use here a slightly different notation than in Section 4). Let us suppose that $M\left(V_{n}\right)=0$; this means that $\int_{\partial B_{R}} V_{n} x_{j} x_{k} d \sigma=0$, for all $j, k=1, \ldots, d$, and in particular $V_{n}$ is orthogonal to $\mathcal{H}_{2}$.

If we assume now that $V_{n}$ is orthogonal to $\mathcal{H}_{2}$, using that

$$
\mathcal{H}_{2}=\operatorname{span}\left\{x_{j} x_{k}, j \neq k \in\{1, \ldots, d\}, x_{1}^{2}-x_{j}^{2}, j=2, \ldots, d\right\}
$$

and moreover that $\int_{\partial B_{R}} V_{n}=0$, we obtain

$$
d \int_{\partial B_{R}} V_{n} x_{1}^{2}=\sum_{j=2_{\partial B_{R}}}^{d} V_{n}\left(x_{1}^{2}-x_{j}^{2}\right)+\int_{\partial B_{R}} \sum_{j=1}^{d} x_{j}^{2}=0,
$$

and therefore

$$
\int_{\partial B_{R}} V_{n} x_{j}^{2}=\int_{\partial B_{R}} V_{n}\left(x_{j}^{2}-x_{1}^{2}\right)=0
$$

which concludes the proof.

In the case where $M_{B_{R}}\left(V_{n}\right) \neq 0$, we compute the trace of the matrix $M_{B_{R}}\left(V_{n}\right)$ to obtain information on its eigenvalues.

Proposition 3.10. When $\Omega$ is a ball of radius $R$, then

$$
\operatorname{Tr}\left(M_{B_{R}}\left(V_{n}\right)\right)=0
$$

for all volume preserving deformations.

Proof. It comes that

$$
\operatorname{Tr}\left(M_{B_{R}}\left(V_{n}\right)\right)=-C(d, R) \int_{\partial B_{R}} \sum_{j=1}^{d} x_{j}^{2} V_{n} d \sigma=-C(d, R) \sum_{j=1}^{d} x_{j}^{2} \int_{\partial B_{R}} V_{n} d \sigma=0
$$

since we are concerned with deformations preserving the volume.

As a consequence of Proposition 3.9 and Proposition 3.10, there is the following alternative: either the only eigenvalue of $M\left(V_{n}\right)$ is 0 , or $M\left(V_{n}\right)$ has at least one nonnegative and one nonpositive eigenvalue. Each $t \mapsto \lambda_{i}(t)$ given by Theorem 3.4 has a directional derivative at $t=0$ denoted by $\lambda_{i}^{\prime}(0)$. We then define, as usual [8], $\partial \lambda_{1}$ the subgradient of $\lambda_{1}$ by $\partial \lambda_{1}=\left[\inf _{i=1 \cdots d} \lambda_{i}^{\prime}(0), \sup _{i=1 \cdots d} \lambda_{i}^{\prime}(0)\right]$. With this notation, $0 \in \partial \lambda_{1}$ and we say the ball is a critical shape.

\subsection{Numerical illustrations}

In order to illustrate Proposition 3.10, we consider the two dimensional case and consider perturbations of the disk given in polar coordinates by

$$
\rho_{t}(\theta)=R+t f(\theta)
$$

where $f$ has zero mean value.

In Fig. 5, the computations are made in the case $R=1$ and $\beta=10$, the deformation parameter $t$ appears in the abscissa.

In both collection of figures, we can see the derivatives of the second and third eigenvalues vanish at the ball in every case except when $f(\theta)=\cos (2 \theta)$, where the regular lines cross, leading to a really nondifferentiable second eigenvalue. This is coherent with Proposition 3.9. Let us explicit the case $V_{n}=R^{2} \cos 2 \theta$, where we are led to compute the eigenvalues of the following symmetric matrix

$$
M=-\frac{3}{\pi R}\left(\begin{array}{cc}
\int_{0}^{2 \pi} \cos 2 \theta \cos ^{2} \theta d \theta & 0 \\
0 & \int_{0}^{2 \pi} \cos 2 \theta \sin ^{2} \theta d \theta
\end{array}\right)
$$

whose eigenvalues are $\alpha_{1}=-\frac{3}{2 R}$ and $\alpha_{2}=\frac{3}{2 R}$. 


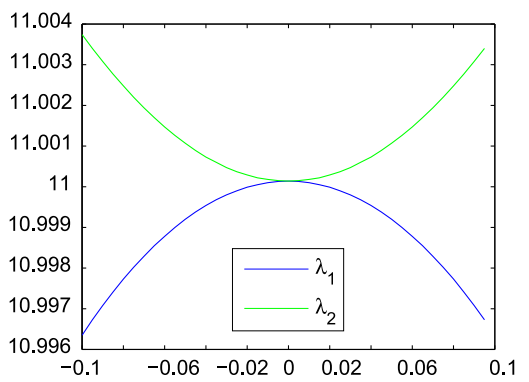

(a) $f(\theta)=\cos (\theta)$

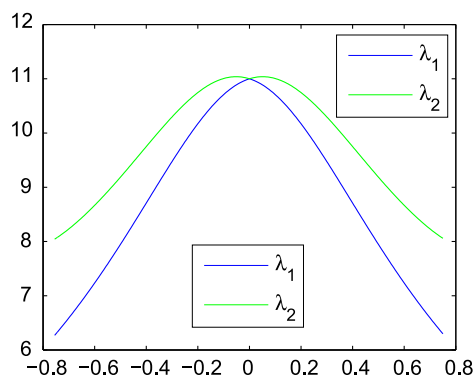

(d) $f(\theta)=\sin (2 \theta)$

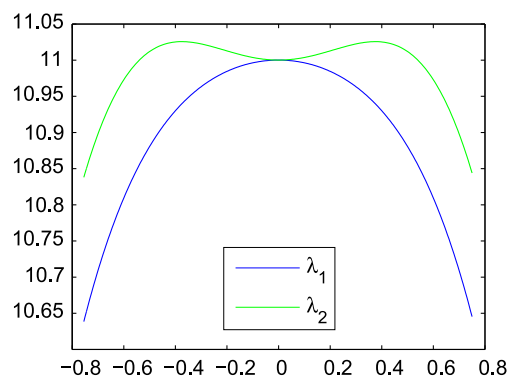

(b) $f(\theta)=\sin (\theta)$

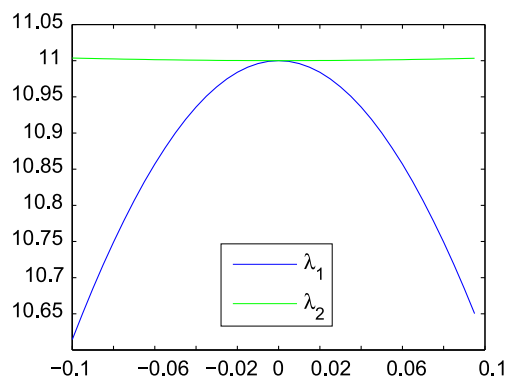

(e) $f(\theta)=\cos (3 \theta)$

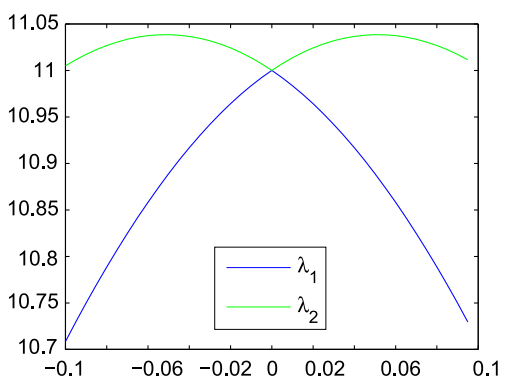

(c) $f(\theta)=\cos (2 \theta)$

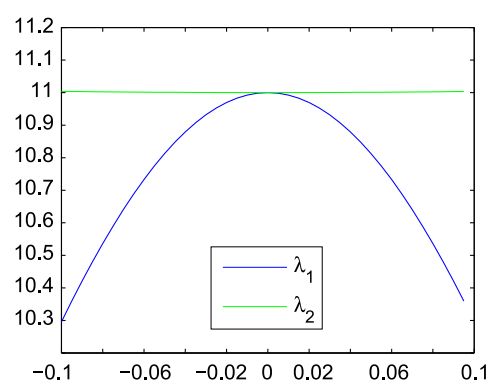

(f) $f(\theta)=\cos (4 \theta)$

Fig. 5. $\lambda_{1}(\Omega)$ and $\lambda_{2}(\Omega)$ in the direction of $f(\theta)-\left|B_{R}\right|=\pi, \beta=10$. (For interpretation of the references to color in this figure legend, the reader is referred to the web version of this article.)

\section{Testing if the ball is a local maximum for $\lambda_{1}$ : second order arguments}

We know that any ball is a critical point for volume preserving deformations. Therefore, if the subgradient $\partial \lambda_{1}\left(B ; V_{n}\right) \neq\{0\}$, then the ball is a local maximizer. It remains to deal with the case where all the eigenvalues of $M_{B}\left(V_{n}\right)$ are 0 ; this case corresponds to $V_{n}$ orthogonal to the harmonics of order two. Then, we aim at proving that the second derivative of $\lambda_{1}$ along at least one of the smooth branches is nonpositive.

The necessary order two conditions of optimality are: the second derivative of the Lagrangian should be nonpositive on the subspace orthogonal to the space generated by the gradient of the volume constraint. We compute:

$$
\operatorname{Vol}^{\prime}(0)=\int_{\partial B_{R}} V_{n}
$$

Hence $\operatorname{Vol}^{\prime}(0)=0$ if and only if $V_{n} \in\left(\mathcal{H}_{0}\right)^{\perp}$ where $\mathcal{H}_{k}$ denotes the linear space of spherical harmonics of order $k$. Due to the previous remarks, we hence consider deformation field in the hilbertian space $\mathcal{H}$ spanned by all the spherical harmonics of order $l \in I=\mathbb{N} \backslash\{0,2\}$. The normal component of such a field is orthogonal to spherical harmonics of order 0 and 2.

The goal of this section is to present the different steps for the computations. We will characterize the matrix $E$ whose eigenvalues are the second order derivatives of the smooth branches of eigenvalues. It turns out that this computation is hard even in the case of a ball. Nevertheless, the computation of $\operatorname{Tr}(E)$ is much simpler than the individual computations of the entries. In order to prove that the ball is a local maximum of $\lambda_{1}$, it suffices to prove that its trace is nonpositive: therefore at least one smooth branch of eigenvalues has a nonpositive second order derivative.

In this section, we consider deformations preserving the volume at second order and not only at first order. Hence, we cannot consider deformation $T_{t}$ of type $I+t V$ with $V$ independent of $t$ and introduce deformations $S_{t}$ that are the flow at time $t$ of a vector field $V$ (see also Remark 1.5). Notice that $S_{t}=I+t V+o(t)$ so that $T_{t}-S_{t}=o(t)$ and first order shape derivatives are unchanged. In particular, one has

$$
\frac{d^{2}}{d t^{2}} \operatorname{Vol}\left(S_{t}(\Omega)\right)=\int_{\partial \Omega(t)}\left(\frac{\partial}{\partial t}\left(V_{n(t)}\right)+V_{n(t)} \frac{\partial}{\partial n(t)}\left(V_{n(t)}\right)+H V_{n(t)}^{2}\right) d \sigma
$$


and the volume preservation at second order means that

$$
\left.\left(\frac{d^{2}}{d t^{2}} \operatorname{Vol}\left(S_{t}(\Omega)\right)\right)\right|_{t=0}=\left.\int_{\partial \Omega}\left(\frac{\partial}{\partial t}\left(V_{n(t)}\right)+V_{n(t)} \frac{\partial}{\partial n(t)}\left(V_{n(t)}\right)+H V_{n(t)}^{2}\right)\right|_{t=0} d \sigma=0 .
$$

\subsection{Construction of the matrix $E$ of the second derivatives}

Let $\left(u(t, x), \lambda(t)=\lambda\left(\Omega_{t}\right)\right)$ be an eigenpair of the Laplace-Wentzell problem, that is to say it solves

$$
\begin{cases}\Delta u(t, x)=0 & \text { in } \Omega_{t} \\ -\beta \Delta_{\tau} u(t, x)+\partial_{n} u(t, x)=\lambda(t) u(t, x) & \text { on } \partial \Omega_{t}\end{cases}
$$

We use the decomposition of $u=u(0, x)$ in the basis of eigenfunctions:

$$
u=\sum_{j=1}^{d} c_{j} u_{j}
$$

for some $c_{1}, c_{2}, \ldots, c_{d}$ not all zero. We have shown that the vector $c=\left(c_{1}, c_{2}, \ldots, c_{d}\right)^{T}$ is solution of

$$
\lambda^{\prime}(0) c=M\left(V_{n}\right) c
$$

where the matrix $M\left(V_{n}\right)=\left(M_{j k}\right)_{1 \leq i, j \leq d}$ is defined by (37).

To compute the second derivative at $t=0$, one has to compute the first shape derivative $u^{\prime}(x)=u^{\prime}(0, x)$. Fredholm's alternative insures the existence of a unique harmonic function $\tilde{u}_{j}$ orthogonal to the eigenfunctions $u_{1}, u_{2}, \ldots, u_{d}$ and satisfying on $\partial \Omega$ the boundary condition

$$
\begin{aligned}
-\beta \Delta_{\tau} \tilde{u}_{j}+\partial_{n} \tilde{u}_{j}-\lambda \tilde{u}_{j}= & \beta\left[\Delta_{\tau}\left[V_{n} \partial_{n} u_{j}\right]+\operatorname{div}_{\tau}\left[V_{n}\left(H I_{d}-2 D^{2} b\right) \cdot \nabla_{\tau} u_{j}\right]\right] \\
& +\operatorname{div}_{\tau}\left[V_{n} \nabla_{\tau} u_{j}\right]+\lambda^{\prime} u_{j}+\lambda V_{n}\left(\partial_{n} u_{j}+H u_{j}\right) .
\end{aligned}
$$

It follows that

$$
u^{\prime}=\sum_{j=1}^{d} \tilde{c}_{j} u_{j}+\sum_{j=1}^{d} c_{j} \tilde{u}_{j}
$$

for some $c_{j}, \tilde{c}_{j}$ when $j=1, \ldots, d$. We point out that the $\left(c_{j}\right)$ are the same coefficients as the decomposition of $u$ in the basis $\left(u_{j}\right)$.

The strategy is straightforward: we have to consider the equation satisfied by $u^{\prime}$ on the boundary $\partial \Omega$ and take its shape derivative again. A first look at the second derivative shows that we will encounter three operators:

- the first contains only $u^{\prime \prime}$ and its expression is the following

$$
E^{(0)}=-\beta \Delta u^{\prime \prime}+\partial_{n} u^{\prime \prime}-\lambda u^{\prime \prime}
$$

- concerning the term in $u^{\prime}$ and $\lambda^{\prime}=0$ we have

$$
E^{(1)}=-2 \beta \Delta_{\tau}\left(V_{n} \partial_{n} u^{\prime}\right)-2 \operatorname{div}_{\tau}\left(V_{n}(I+\beta \mathcal{A}) \nabla_{\tau} u^{\prime}\right)-2\left[\lambda^{\prime} u^{\prime}+\lambda V_{n}\left(\partial_{n} u^{\prime}+H u^{\prime}\right)\right]
$$

where $\mathcal{A}=H I-2 D^{2} b$ is the deviatoric part of the curvature tensor.

- The remaining term $E^{(2)}$ contains only $u$; we give a more explicit expression below.

Green-Riemann identity tells us that $\left\langle E^{(0)}, u_{i}\right\rangle=\left\langle u^{\prime \prime},-\beta \Delta_{\tau} u_{i}+\partial_{n} u_{i}-\lambda u_{i}\right\rangle=0, i=1, \ldots, d$. This means that the term $E^{(0)}$ will have no influence on the determination of the second derivative of the eigenvalue. We will focus only on $E^{(1)}$ and $E^{(2)}$.

Construction of $E^{(2)}$ : The computations are very technical. We need first to use a test function $\phi$ which is the restriction of a test function $\Phi$ defined on a tubular neighborhood of the boundary such that its normal derivative 
on $\partial \Omega$ is zero. This kind of extension is well discussed in the book [13] of Delfour and Zolésio. Taking the shape derivative of the boundary condition (36) (in the multiple case) we need to compute

$$
\begin{aligned}
& \left.\left(\frac{d}{d t} \int_{\partial \Omega_{t}} V_{n} \nabla_{\tau} u \cdot \nabla_{\tau} \phi d \sigma_{t}\right)\right|_{t=0}=\left\langle A^{(1)} u^{\prime}, \phi\right\rangle+\left\langle A^{(2)} u, \phi\right\rangle, \\
& \left.\beta\left(\frac{d}{d t} \int_{\partial \Omega_{t}} \mathcal{A}(t) V_{n} \nabla_{\tau} u \cdot \nabla_{\tau} \phi d \sigma_{t}\right)\right|_{t=0}=\left\langle B^{(1)} u^{\prime}, \phi\right\rangle+\left\langle B^{(2)} u, \phi\right\rangle, \\
& -\left.\frac{d}{d t}\left(\int_{\partial \Omega_{t}}\left[\lambda^{\prime} u+\lambda\left(u^{\prime}+V_{n} \partial_{n} u+V_{n} H u\right)\right] \phi d \sigma_{t}\right)\right|_{t=0}=\left\langle C^{(0)} u^{\prime \prime}, \phi\right\rangle+\left\langle C^{(1)} u^{\prime}, \phi\right\rangle+\left\langle C^{(2)} u, \phi\right\rangle, \\
& \left.\beta\left(\frac{d}{d t} \int_{\partial \Omega_{t}} \nabla_{\tau}\left(V_{n} \nabla_{\tau} \partial_{n} u\right) . \nabla_{\tau} \phi d \sigma_{t}\right)\right|_{t=0}=\left\langle D^{(1)} u^{\prime}, \phi\right\rangle+\left\langle D^{(2)} u, \phi\right\rangle .
\end{aligned}
$$

The remaining $E^{(2)}$ containing only $u$ is then given by

$$
E^{(2)}=A^{(2)} u+B^{(2)} u+C^{(2)} u+D^{(2)} u .
$$

For an operator $L$ involved in $E^{(i)}, i=1,2,3$, we denote by $\left(L_{i j}\right)_{i, j=1, \ldots, d}$ the matrix of $L$ in the basis of the eigenvalues. After calculations (see also Remark D.1 in Appendix D), we get the following linear equation

$$
\left(\lambda^{\prime \prime} I-E\right) c+2\left(-M\left(V_{n}\right)+\lambda^{\prime} I\right) \tilde{c}=0
$$

(corresponding to the second derivation) together with

$$
\left(-M\left(V_{n}\right)+\lambda^{\prime} I\right) c=0
$$

(corresponding to the first derivation) where the matrix $E=\left(E_{i j}\right)$ is split into $E=E^{(1)}+E^{(2)}$ where the terms involving $u^{\prime}$ are gathered in $E^{(1)}$ and the terms involving $u$ are gathered in $E^{(2)}$.

\subsection{Computation of the trace}

Since the direct computations of the eigenvalues are difficult, we restrict ourselves to the cases $d=2$ or $d=3$, and we will focus on the trace of $E$ and prove that $\operatorname{Tr}(E)$ is nonpositive. We start with the trace of $E^{(2)}$ :

Lemma 4.1. Assume $d \in\{2,3\}$. With $K(R)=\frac{d}{R^{2+d} \omega_{d-1}}$, we have

$$
\operatorname{Tr}\left(E^{(2)}\right)=-(d \beta+R) R K(R) \int_{\partial B_{R}}\left|\nabla_{\tau} V_{n}\right|^{2} d \sigma-K(R) \int_{\partial B_{R}} V_{n}^{2} d \sigma
$$

for all deformations preserving volume and such that $V_{n}$ is orthogonal to spherical harmonics of order two.

Proof. The computation of $E^{(2)}$ is done in the Appendix C, and to obtain the result, we sum all the traces given by Lemmas C.1, C.2, C.3 and C.4.

Concerning $\operatorname{Tr}\left(E^{(1)}\right)$, we start with the following lemma which is straightforward (see also Remark D.1):

Lemma 4.2. We have that

$$
\operatorname{Tr}\left(E^{(1)}\right)=2 \int_{\partial \Omega} V_{n} \sum_{j=1}^{d}\left(-\partial_{n} \tilde{u}_{j} \partial_{n} u_{j}-H \lambda \tilde{u}_{j} u_{j}+\left(I+\beta\left(H I_{d}-2 D^{2} b\right)\right) \nabla_{\tau} \tilde{u}_{j} . \nabla_{\tau} u_{j}\right) d \sigma
$$

holds for all deformations preserving volumes such that $V_{n}$ is orthogonal to spherical harmonics of order two.

From this result we deduce the following, which is proved in Appendix D: 
Proposition 4.3. Assume $d=3$ and set $\alpha=\frac{\beta}{R}$. We denote by $Y_{l}^{m}, m=-l, \ldots, m$, any spherical harmonic of order $l \in I$. If

$$
V_{n}=\sum_{l \in I} R^{l}\left(\sum_{m=-l}^{l} v_{l, m} Y_{l}^{m}\right)
$$

then

$$
\operatorname{Tr}\left(E^{(1)}\right)=-K(R)\left(\sum_{l \in I}\left[A_{l, \alpha}+B_{l, \alpha}\right] R^{2 l+1} \sum_{m=-l}^{l}\left|v_{l, m}\right|^{2}\right)
$$

where

$$
A_{l, \alpha}=\frac{l}{2 l+1} \frac{l+2}{l-2}(4 \alpha+2 l) \frac{1+\alpha(3-l)}{1+\alpha(l+1)} \quad \text { and } \quad B_{l, \alpha}=\frac{l+1}{l} \frac{l-1}{l}(4 \alpha+2) \frac{1+\alpha(4+l)}{1+\alpha(3+l)} .
$$

Since $\operatorname{Tr} E=\operatorname{Tr}\left(E^{(1)}\right)+\operatorname{Tr}\left(E^{(2)}\right)$, we will then deduce the following result:

Proposition 4.4. Assume $d \in\{2,3\}$. Then there exists a nonnegative constant $\mu$ such that

$$
\operatorname{Tr}(E) \leq-K(R) \mu \int_{\partial B_{R}}\left|\nabla_{\tau} V_{n}\right|^{2}+\left|V_{n}\right|^{2} d \sigma
$$

holds for all preserving volume deformations such that $V_{n}$ is orthogonal to $\mathcal{H}$.

Proof. We distinguish the cases $d=2$ and $d=3$.

The case $d=2$. Let us compute the trace of the matrix $E$. Gathering all the results of Lemma 4.1 with the computations of Appendix D concerning the trace of the different matrices involved in the matrix $E$, we obtain the following formula: when

$$
V_{n}=\sum_{l \in I} \frac{R^{l}}{\sqrt{\pi}}\left(v_{1}^{(l)} \cos l \theta+v_{2}^{(l)} \sin l \theta\right), \quad l \in I,
$$

we have

$$
\operatorname{Tr}(E)=-K(R) \sum_{l \in I} G(\alpha, l)\left(l^{2}+1\right) R^{2 l+1}\left(\left(v_{1}^{(l)}\right)^{2}+\left(v_{1}^{(l)}\right)^{2}\right),
$$

where

$$
G(\alpha, l)=\frac{\left(l^{2}-1\right)}{2\left(1+l^{2}\right)} \frac{2+l^{2}+2 \alpha^{2}(l-2) l^{2}+\alpha(l-2)\left(l^{2}+2\right)}{(l-2) l(1+\alpha l)} .
$$

Let us remark that $G(\alpha, 1)=0$. This could have been guessed since the Wentzell eigenvalues are translation invariance: we recall that, denoting by Bar the center of mass of the boundary, we have

$$
\operatorname{Bar}^{\prime}(0)=\int_{\partial B_{R}} x V_{n}
$$

so that deformations orthogonal to spherical harmonics of order 1 preserve at first order the center of mass. A close look at the fraction $G$ shows that it has no pole for $\alpha>0$ and $l \geq 3$, that it is nonnegative for $l>2$ and that $G(l, \alpha) \rightarrow 1$ when $l \rightarrow+\infty$; then there is a nonnegative constant $\mu$ such that for all $l \geq 3, \mu \leq G(l, \alpha)$. This gives

$$
\operatorname{Tr}(E) \leq-K(R) \mu \int_{\partial B_{R}}\left|\nabla_{\tau} V_{n}\right|^{2}+\left|V_{n}\right|^{2} d \sigma .
$$

The case $d=3$. The strategy is the same, and we use again Lemma 4.1 and the detailed computations from Section D.2: we get for $l \in I$ : 


$$
\begin{aligned}
& V_{n}=\sum_{l \in I} R^{l} \sum_{p=-l}^{l} v_{p}^{(l)} Y_{l}^{p}, \\
& \operatorname{Tr}(E)=-K(R) \sum_{l \in I} F(\alpha, l)(l(l+1)+1) R^{2 l+1} \sum_{p=-l}^{l}\left(v_{p}^{(l)}\right)^{2},
\end{aligned}
$$

where $F(\alpha, l)$ is the fraction

$$
F(\alpha, l)=\frac{(l-1) \sum_{m=0}^{3} P_{m}(l) \alpha^{m}}{(l(l+1)+1) l(1+\alpha(l+1))(2 l+1)(l-2)(1+\alpha(l+3))},
$$

and where the polynomials $P_{m}$ are defined as

$$
\begin{aligned}
& P_{0}(X)=2 X^{4}+5 X^{3}+16 X^{2}-8, \\
& P_{1}(X)=4 X^{5}+18 X^{4}+40 X^{3}+68 X^{2}-28 X-56, \\
& P_{2}(X)=2 X^{6}+21 X^{5}+42 X^{4}+35 X^{3}+16 X-112, \\
& P_{3}(X)=8 X^{6}+18 X^{5}+24 X^{4}-68 X^{3}-144 X^{2}-112 X-64 .
\end{aligned}
$$

Let us remark that $F(\alpha, 1)=0$ for the same reason as in dimension two. By Descartes's rule of signs, the polynomials $P_{m}$ have at most one positive root. Since $P_{m}(0)<0$ and $P_{m}(2)>0$ for $m=0, \ldots 3, P_{m}$ has exactly one positive root which is in $[0,2]$. Since $l>2$, there exists a nonnegative constant $\mu$ such that for all $k \geq 3, \mu \leq F(k, \alpha)$ and

$$
\operatorname{Tr}(E) \leq-K(R) \mu \int_{\partial B_{R}}\left|\nabla_{\tau} V_{n}\right|^{2}+\left|V_{n}\right|^{2} d \sigma .
$$

\section{Conflict of interest statement}

The authors certify that they have no affiliations with or involvement in any organization or entity with any financial interest, or non-financial interest in the subject matter or materials discussed in this manuscript.

\section{Acknowledgements}

Part of the work was supported by the Projet ANR-12-BS01-0007 OPTIFORM financed by the French Agence Nationale de la Recherche (ANR). We would also like to thank the anonymous reviewer for its careful reading of the previous version of the manuscript, that helped to improve and clarify the paper.

\section{Appendix A. Some classical results on tangential differential calculus}

We recall some facts about tangential operators acting on functions defined on $\partial \Omega$. The formulas involve the extensions of functions and the differential calculus becomes easier since we will use the classical euclidean differential calculus in a neighborhood of $\partial \Omega$. The canonical extension will be provided thanks to the oriented distance and the orthogonal projection on the tangent plane. For more details, the interested reader will consult the book [13] of M. Delfour and J.P. Zolésio from which we borrowed the necessary material.

\section{A.1. Notations and definitions. Preliminary results}

We recall some essential notations and definitions that are needed for the computations of shape derivatives. Given a smooth function $f: \partial \Omega \mapsto \mathbb{R}$, we define its tangential gradient $\nabla_{\tau}$ as

$$
\nabla_{\tau} f=\nabla \tilde{f}-\nabla \tilde{f} . \mathbf{n} \mathbf{n}
$$

where $\tilde{f}$ is any extension of $f$ in a tubular neighborhood of $\partial \Omega$. An extension is easily obtained when $\partial \Omega$ is smooth. The tangential gradient does not depend on the extension. 
It is also useful to define the tangential gradient as the normal projection of $\nabla \tilde{f}$ to the tangent hyperplane of $\partial \Omega$; in other words

$$
\nabla_{\tau} f=\nabla \tilde{f}-n \otimes n \nabla \tilde{f}, \quad \text { on } \partial \Omega .
$$

We also need the definition of the tangential divergence: for a tensor $v$, we define the surface divergence as

$$
\operatorname{div}_{\tau} u=\operatorname{Tr}\left(\nabla_{\tau} u\right)
$$

For regular functions we define the surface Laplacian or Laplace-Beltrami operator as

$$
\Delta_{\tau} f:=\operatorname{div}_{\tau}\left(\nabla_{\tau} f\right)
$$

We recall the definition of the oriented distance $b_{\partial \Omega}$ :

$$
b_{\partial \Omega}(x)= \begin{cases}d_{\Omega}(x) & \text { for } x \in \mathbb{R}^{d} \backslash \bar{\Omega} \\ -d_{\Omega}(x) & \text { for } x \in \Omega\end{cases}
$$

where the notation $d_{\Omega}$ stands for the distance function for a subset $\Omega \subset \mathbb{R}^{d}$ :

$$
d_{\Omega}(x)=\inf _{y \in \Omega}|x-y|
$$

We shall sometimes write $b$ instead of $b_{\partial \Omega}$; its gradient is an extension of the normal vector field $\mathbf{n}$ in a neighborhood of $\partial \Omega$.

Let $D^{2} b$ be the Weingarten operator with entries $\left(\nabla_{\tau}\right)_{i} n_{j}$ where $n_{j}$ is the $j$-th component of $\mathbf{n}$. The normal vector is known to be in the kernel of $D^{2} b$, while the other eigenfunctions are tangential with the corresponding eigenvalues given by the principal curvatures of $\partial \Omega$.

Let $\kappa_{i}, i=1, \ldots, d-1$, be the nonzero eigenvalues of $D^{2} b$. We define the mean curvature $H$ as

$$
H=\sum_{i=1}^{d-1} \kappa_{i}=\operatorname{Tr}\left(D^{2} b\right)=\Delta b, \quad \text { on } \partial \Omega .
$$

An important result about the normal derivative of these quantities is:

Proposition A.1. Suppose that the boundary $\partial \Omega$ is of class $C^{3}$. Then the normal derivative of the mean curvature $H$ is

$$
\partial_{n} H=-\sum_{i=1}^{d-1} \kappa_{i}^{2}
$$

Other known identities: we denote by $\mathbf{x}$ the identity function. We have

$$
\begin{aligned}
& -\Delta_{\tau} \mathbf{x}=H \mathbf{n} \\
& \operatorname{div}_{\tau} \mathbf{n}=H \mathbf{n}
\end{aligned}
$$

Tangential integral formula: Given two functions $f$ (scalar) and $\mathbf{v}$ smooth enough, we have

$$
\int_{\partial \Omega} f \operatorname{div}_{\tau} \mathbf{v}+\int_{\partial \Omega} \nabla_{\tau} f . v=\int_{\partial \Omega} H f \mathbf{v} . \mathbf{n}
$$

Shape derivative of the main curvature $H$ and of the normal $\mathbf{n}$ in the direction of a velocity $\boldsymbol{V}$ :

Proposition A.2. Let a surface $\partial \Omega$ be of class $C^{2}$. The shape derivatives of the normal $\mathbf{n}$ and of the mean curvature $H$ in the direction of the velocity vector $\boldsymbol{V}$ are

$$
\begin{aligned}
& \mathbf{n}^{\prime}=-\nabla_{\tau} V_{n} \\
& H^{\prime}=-\Delta_{\tau} V_{n}
\end{aligned}
$$

where $V_{n}=\langle\boldsymbol{V}, \boldsymbol{n}\rangle$ denotes the normal component of the vector deformation $\mathbf{V}$. 


\section{A.2. A commutation lemma}

Here $f$ and $g$ are two smooth functions defined on $\mathcal{U}$ a neighborhood of $\partial \Omega$; the notation $b$ stands for the oriented distance. Recall that its gradient is an extension of the normal field $\mathbf{n}$ on $\partial \Omega$.

Proposition A.3. We have

$$
\partial_{\mathbf{n}}\left(\nabla_{\tau} f \cdot \nabla_{\tau} g\right)+2\left(D^{2} b \nabla_{\tau} f\right) \cdot \nabla g=\nabla_{\tau}\left(\partial_{\mathbf{n}} f\right) \cdot \nabla_{\tau} g+\nabla_{\tau}\left(\partial_{\mathbf{n}} g\right) . \nabla_{\tau} f
$$

Proof. A straightforward computation gives

$$
\partial_{\mathbf{n}}(\nabla f . \nabla g)=\left(D^{2} f \nabla g\right) \cdot \mathbf{n}+\left(D^{2} g \nabla f\right) \cdot \mathbf{n}
$$

and

$$
\begin{aligned}
\nabla\left(\partial_{\mathbf{n}} f\right) \cdot \nabla g & =\nabla(\nabla f \cdot \mathbf{n}) \cdot \nabla g \\
& =\left(D^{2} f \mathbf{n}\right) \cdot \nabla g+\left(D^{2} b \nabla f\right) \cdot \nabla g
\end{aligned}
$$

hence

$$
\begin{aligned}
\nabla\left(\partial_{\mathbf{n}} f\right) \cdot \nabla g+\nabla\left(\partial_{\mathbf{n}} g\right) \cdot \nabla f & =2\left(D^{2} b \nabla f\right) \cdot \nabla g+\left(D^{2} f \mathbf{n}\right) \cdot \nabla g+\left(D^{2} g \mathbf{n}\right) \cdot \nabla f \\
& =2\left(D^{2} b \nabla f\right) \cdot \nabla g+\partial_{\mathbf{n}}(\nabla f \cdot \nabla g)
\end{aligned}
$$

We use now the decomposition of $\nabla$ into its normal and tangential components and the well known identity $D^{2} b \mathbf{n} . \mathbf{n}=0$. We get

$$
\begin{aligned}
& \nabla_{\tau}\left(\partial_{\mathbf{n}} f\right) \cdot \nabla_{\tau} g+\nabla_{\tau}\left(\partial_{\mathbf{n}} g\right) \cdot \nabla_{\tau} f+\frac{\partial^{2} f}{\partial n^{2}} \frac{\partial g}{\partial n}+\frac{\partial^{2} g}{\partial n^{2}} \frac{\partial f}{\partial n} \\
& =2\left(D^{2} b \nabla_{\tau} f\right) \cdot \nabla_{\tau} g+\partial_{\mathbf{n}}\left(\nabla_{\tau} f \cdot \nabla_{\tau} g\right)+\frac{\partial^{2} f}{\partial n^{2}} \frac{\partial g}{\partial n}+\frac{\partial^{2} g}{\partial n^{2}} \frac{\partial f}{\partial n}
\end{aligned}
$$

hence

$$
\nabla_{\tau}\left(\partial_{\mathbf{n}} f\right) \cdot \nabla_{\tau} g+\nabla_{\tau}\left(\partial_{\mathbf{n}} g\right) \cdot \nabla_{\tau} f=2\left(D^{2} b \nabla_{\tau} f\right) \cdot \nabla_{\tau} g+\partial_{\mathbf{n}}\left(\nabla_{\tau} f . \nabla_{\tau} g\right)
$$

\section{Appendix B. Spherical harmonics}

In order to explicit the shape hessian under consideration, a useful tool is the surface spherical harmonics defined as the restriction to the surface of the unit sphere of harmonic polynomials in the special case $d=3$. We recall here facts from [25, pages 139-141]. Spherical harmonics are defined as restrictions of homogeneous harmonic polynomials to the unit sphere. The spherical harmonics are said to be of order $k$ when the harmonic homogeneous polynomial is of degree $k$. We denote by $\mathcal{H}_{k}$ the space of spherical harmonics of degree $k$. We show that is also the eigenspace of the Laplace-Beltrami operator on the unit sphere associated with the eigenvalue $k(k+1)$. Its dimension is

$$
d_{k}=2 k+1 .
$$

Let $\left(Y_{k}^{l}\right)_{-k \leq l \leq k}$ be an orthonormal basis of $\mathcal{H}_{k}$ with respect to the $\mathrm{L}^{2}\left(\partial B_{1}\right)$ scalar product. The $\left(\mathcal{H}_{k}\right)_{k \in \mathbb{N}}$ spans a vector space dense in $\mathrm{L}^{2}\left(\partial B_{1}\right)$ and the family $\left(Y_{k}^{l}\right)_{k \in \mathbb{N},-k \leq l \leq k}$ is a Hilbert basis of $\mathrm{L}^{2}\left(\partial B_{1}\right)$. To be more precise, if $f \in \mathrm{L}^{2}\left(\partial B_{1}\right)$, then there exists a unique representation

$$
f=\sum_{k=0}^{\infty} \mathbf{Y}_{k}
$$

where the series converge to $f$ in the $\mathrm{L}^{2}$ norm and

$$
\mathbf{Y}_{k}=\sum_{l=-k}^{k} b_{k}^{l} Y_{k}^{l} \in \mathcal{H}_{k}
$$


If $x=\left(x_{1}, x_{2}, x_{3}\right) \in \mathbb{R}^{3}$, it is natural to use on a sphere the spherical coordinates $(r, \theta, \phi)$ where $r$ is the radius and $\theta$ and $\phi$ are the Euler angles. The spherical harmonic $Y_{k}^{l}$ is defined with the Euler angles $(\theta, \phi)$ as

$$
Y_{k}^{l}=(-1)^{l} \sqrt{\left[\frac{k+\frac{1}{2}}{2 \pi} \frac{(k-l) !}{(k+l) !}\right]} e^{i l \phi} \mathbb{P}_{k}^{l}(\cos \theta), \quad-k \leq l \leq k,
$$

where the polynomial $\mathbb{P}_{k}^{l}$ is the associated Legendre polynomial. The formula giving the explicit form of these polynomials can be found in the book of Nédélec [23, page 24].

When $k \neq k^{\prime}$, we have also the orthogonality property

$$
\int_{\partial B_{1}} \mathbf{Y}_{k} \mathbf{Y}_{k^{\prime}} d \sigma=0
$$

when $\mathbf{Y}_{k} \in \mathcal{H}_{k}$ and $\mathbf{Y}_{k^{\prime}} \in \mathcal{H}_{k^{\prime}}$. A homogeneity argument shows that any function $\varphi$ in $L^{2}\left(\partial B_{R}\right)$ can be decomposed as the Fourier series:

$$
\varphi(x)=\sum_{k=0}^{\infty} R^{k}\left(\sum_{l=-k}^{k} \alpha_{k, l}(\varphi) Y_{k}^{l}\left(\frac{x}{|x|}\right)\right), \quad \text { for }|x|=R .
$$

Then, by construction, the function $u$ defined by

$$
u(x)=\sum_{k=0}^{\infty}|x|^{k}\left(\sum_{l=1}^{d_{k}} \alpha_{k, l}(\varphi) Y_{k}^{l}\left(\frac{x}{|x|}\right)\right), \quad \text { for }|x| \leq R,
$$

being harmonic in $B_{R}$ and satisfying $u=\varphi$ on $\partial B_{R}$.

We recall now some results about the integration of three spherical harmonics, they will enable us to estimate $\operatorname{Tr}(E)$ in dimension three. When we integrate three spherical harmonics, we use coefficients called Clebsch-Gordon coefficients or Wigner- $3 j$ coefficients. The Wigner-3 $j$ coefficients are mostly used; they are related to ClebschGordon coefficients via some known formula that the interested reader will find in the book of Cohen Tannoudji et al. [9, Tome 2, Annex B].

The first general result concerns the product of two spherical harmonics; it is given by the following proposition.

Proposition B.1. Given $l_{1}, l_{2}>0$ two natural integers and $-l_{1} \leq m_{1} \leq l_{1},-l_{2} \leq m_{2} \leq l_{2}$, we have

$$
Y_{l_{1}}^{m_{1}} Y_{l_{2}}^{m_{2}}=(-1)^{m_{1}+m_{2}} \sum_{L=\left|l_{1}-l_{2}\right|}^{l_{1}+l_{2}} \sqrt{\frac{\left(2 l_{1}+1\right)\left(2 l_{2}+1\right)(2 L+1)}{4 \pi}}\left(\begin{array}{ccc}
l_{1} & l_{2} & L \\
0 & 0 & 0
\end{array}\right)\left(\begin{array}{ccc}
l_{1} & l_{2} & L \\
m_{1} & m_{2} & -m_{1}-m_{2}
\end{array}\right) Y_{L}^{m_{1}+m_{2}},
$$

where $\left(\begin{array}{ccc}l_{1} & l_{2} & L \\ 0 & 0 & 0\end{array}\right)$ and $\left(\begin{array}{ccc}l_{1} & l_{2} & L \\ m_{1} & m_{2} & -m_{1}-m_{2}\end{array}\right)$ are the Wigner-3j symbols.

The second result concerns the integration of three spherical harmonics.

Proposition B.2. We have:

$$
\int_{\partial B_{1}} Y_{l_{1}}^{m_{1}} Y_{l_{2}}^{m_{2}} Y_{l_{3}}^{m_{3}}=\sqrt{\frac{\left(2 l_{1}+1\right)\left(2 l_{2}+1\right)\left(2 l_{3}+1\right)}{4 \pi}}\left(\begin{array}{ccc}
l_{1} & l_{2} & l_{3} \\
0 & 0 & 0
\end{array}\right)\left(\begin{array}{ccc}
l_{1} & l_{2} & l_{3} \\
m_{1} & m_{2} & m_{3}
\end{array}\right) .
$$

In particular it holds

Proposition B.3. Let $l$ be a natural integer and $m$ an integer. We have:

1. If $-l \leq m \leq l$ then

$$
\int_{\partial B_{1}} Y_{l}^{m} Y_{0}^{0} \overline{Y_{l}^{m}}=\sqrt{\frac{1}{4 \pi}}
$$


and

$$
\int_{\partial B_{1}} Y_{l}^{m} Y_{1}^{1} \overline{Y_{l-1}^{m+1}}=-\sqrt{\frac{3}{8 \pi}} \sqrt{\frac{(l-m)(l-m-1)}{(2 l+1)(2 l-1)}} .
$$

2. If $-l-1 \leq m \leq l+1$ then

$$
\int_{\partial B_{1}} Y_{l}^{m} Y_{1}^{0} \overline{Y_{l+1}^{m}}=\sqrt{\frac{3}{4 \pi}} \sqrt{\frac{(l+m+1)(l-m+1)}{(2 l+1)(2 l+3)}} .
$$

3. If $-l-2 \leq m \leq l$ then

$$
\int_{\partial B_{1}} Y_{l}^{m} Y_{1}^{1} \overline{Y_{l+1}^{m+1}}=\sqrt{\frac{3}{8 \pi}} \sqrt{\frac{(l+m+1)(l+m+2)}{(2 l+1)(2 l+3)}} .
$$

\section{Appendix C. Intermediate results for the second shape derivative matrix}

We need to construct the matrix associated to the second shape derivative. To that end, we have to compute the explicit formula for all the shape derivatives of order one involved in the formula giving $\lambda^{\prime}$ (see Theorem 3.6). In this appendix, we focus on the term $E^{(2)}$ introduced in Section 4.1. Since these computations are very technical, we only give the main line and the used arguments, omitting a couple of details. In the following lines, we denote by $H(t)$ the mean curvature associated to the boundary of $\Omega_{t}$ and $\mathcal{A}(t)$ the deviatoric part defined on $\partial \Omega_{t}$ as

$$
\mathcal{A}(t)=H(t) I-2 D^{2} b(t)
$$

(see [13] for the terminology).

In order to deal with the weak formulation on the boundary $\partial \Omega_{t}$, we will make use of a test function $\phi$ which is the restriction of a test function $\Phi$ defined on a tubular neighborhood of the boundary such that its normal derivative is zero. This kind of extension is well discussed in the book [13] of Delfour and Zolésio.

In this differentiation, nineteen terms arise and we introduce some notations to study them separately. For all function test $\phi \in H^{1}(\partial \Omega)$, we will need in the sequel the following quantities:

$$
\begin{aligned}
& A\left(u, u^{\prime}, \phi\right)=\left.\left(\frac{d}{d t} \int_{\partial \Omega_{t}} V_{n} \nabla_{\tau} u \cdot \nabla_{\tau} \phi d \sigma_{t}\right)\right|_{t=0}, \\
& B\left(u, u^{\prime}, \phi\right)=\left.\beta\left(\frac{d}{d t} \int_{\partial \Omega_{t}} \mathcal{A}(t) V_{n} \nabla_{\tau} u \cdot \nabla_{\tau} \phi d \sigma_{t}\right)\right|_{t=0}, \\
& C\left(u, u^{\prime}, u^{\prime \prime}, \phi\right)=-\left.\frac{d}{d t}\left(\int_{\partial \Omega_{t}}\left[\lambda^{\prime} u+\lambda\left(u^{\prime}+V_{n} \partial_{n} u+V_{n} H u\right)\right] \phi d \sigma_{t}\right)\right|_{t=0}, \\
& D\left(u, u^{\prime}, \phi\right)=\left.\beta\left(\frac{d}{d t} \int_{\partial \Omega_{t}} \nabla_{\tau}\left(V_{n} \partial_{n} u\right) . \nabla_{\tau} \phi d \sigma_{t}\right)\right|_{t=0} .
\end{aligned}
$$

We will now study independently each term $A, B, C$ and $D$, when $\Omega=B_{R} \subset \mathbb{R}^{2}$ or $\mathbb{R}^{3}$, and $t \mapsto \Omega_{t}$ is volume preserving.

Study of $D\left(u, u^{\prime}, \phi\right)$. First, we denote

$$
W=\left.\frac{d}{d t}\left(\boldsymbol{V} \cdot \boldsymbol{n}_{\Omega_{t}}\right)\right|_{t=0} .
$$

From the derivative formula of boundary integrals, we know that we have to compute three main terms: the first corresponds to the shape derivative, the second concerns the normal derivative of the integrand and the third is related to the term related to the mean curvature $H$. The first term is 


$$
\begin{aligned}
& \left.\beta\left(\int_{\partial \Omega_{t}} \frac{d}{d t}\left[\nabla_{\tau}\left(V_{n} \partial_{n} u\right) \cdot \nabla_{\tau} \phi\right] d \sigma_{t}\right)\right|_{t=0} \\
& =\beta\left(\int_{\partial B_{R}} \nabla_{\tau}\left(V_{n} \cdot \partial_{n} u^{\prime}-V_{n} \nabla_{\tau} u \cdot \nabla_{\tau} V_{n}\right) \cdot \nabla_{\tau} \phi d \sigma+\int_{\partial B_{R}} \nabla_{\tau}\left(V_{n}^{\prime} \cdot \partial_{n} u\right) \cdot \nabla_{\tau} \phi d \sigma\right) \\
& \quad+\beta \int_{\partial B_{R}} \partial_{n}\left(V_{n} \partial_{n} u\right) \nabla_{\tau} V_{n} \cdot \nabla_{\tau} \phi d \sigma \\
& =-\beta \int_{\partial B_{R}} \Delta_{\tau}\left(V_{n} \cdot \partial_{n} u^{\prime}\right) \phi d \sigma+\beta \int_{\partial B_{R}} \nabla_{\tau}\left(V_{n}^{\prime} \cdot \partial_{n} u\right) \cdot \nabla_{\tau} \phi d \sigma \\
& \quad+\beta \int_{\partial B_{R}} \partial_{n}\left(V_{n} \partial_{n} u\right) \nabla_{\tau} V_{n} \cdot \nabla_{\tau} \phi d \sigma+\beta \int_{\partial B_{R}} \Delta_{\tau}\left(V_{n} \nabla_{\tau} u \cdot \nabla_{\tau} V_{n}\right) \phi d \sigma .
\end{aligned}
$$

The third term is

$$
\beta \int_{\partial B_{R}} H V_{n} \nabla_{\tau}\left(V_{n} \partial_{n} u\right) . \nabla_{\tau} \phi d \sigma=-\beta \int_{\partial B_{R}} \operatorname{div}_{\tau}\left(H V_{n} \nabla_{\tau}\left(V_{n} \partial_{n} u\right)\right) \phi d \sigma .
$$

We focus now on the second term. We have

$$
\begin{aligned}
& \beta \int_{\partial B_{R}} V_{n} \partial_{n}\left[\nabla_{\tau}\left(V_{n} \partial_{n} u\right) \cdot \nabla_{\tau} \phi\right] d \sigma \\
& \quad=\beta \int_{\partial B_{R}} V_{n} \nabla_{\tau}\left[\partial_{n}\left(V_{n} \partial_{n} u\right)\right] \cdot \nabla_{\tau} \phi d \sigma-2 \beta \int_{\partial B_{R}} V_{n}\left(D^{2} b \nabla_{\tau}\left[V_{n} \partial_{n} u\right]\right) \cdot \nabla_{\tau} \phi d \sigma \\
& =\beta\left(\int_{\partial B_{R}} V_{n} \nabla_{\tau}\left[\partial_{n}\left(V_{n} \partial_{n} u\right)\right] \cdot \nabla_{\tau} \phi d \sigma-2 \int_{\partial B_{R}} V_{n}\left(D^{2} b \nabla_{\tau}\left[V_{n} \partial_{n} u\right]\right) \cdot \nabla_{\tau} \phi d \sigma\right) \\
& =-\beta \int_{\partial B_{R}} \operatorname{div}_{\tau}\left[V_{n} \nabla_{\tau}\left[\partial_{n} u \partial_{n} V_{n}\right]-2 V_{n} D^{2} b \nabla_{\tau}\left[V_{n} \partial_{n} u\right]\right] \phi d \sigma .
\end{aligned}
$$

We expand $D(u, \phi)$ into a sum $\left\langle D^{(1)} u^{\prime}, \phi\right\rangle+\left\langle D^{(2)} u, \phi\right\rangle$. For $D^{(2)}$, we will set $D^{(2)}=\sum_{k=1}^{3} D^{(2, k)}$ where

$$
\begin{aligned}
\left\langle D^{(1)} u^{\prime}, \phi\right\rangle= & \beta \int_{\partial B_{R}} \nabla_{\tau}\left[V_{n} \cdot \partial_{n} u^{\prime}\right] \cdot \nabla_{\tau} \phi d \sigma=-\beta \int_{\partial B_{R}} \Delta_{\tau}\left[V_{n} \partial_{n} u^{\prime}\right] \phi d \sigma \\
\left\langle D^{(2,1)} u, \phi\right\rangle= & \beta\left[\int_{\partial B_{R}}-\Delta_{\tau}\left[W \partial_{n} u\right] \phi d \sigma-\int_{\partial B_{R}} \operatorname{div}_{\tau}\left[V_{n} \partial_{n} V_{n} \nabla_{\tau}\left[\partial_{n} u\right]\right] \phi d \sigma\right. \\
& \left.-\int_{\partial B_{R}} \operatorname{div}_{\tau}\left[H V_{n} \nabla_{\tau}\left(V_{n} \partial_{n} u\right)\right] \phi d \sigma\right] \\
\left\langle D^{(2,2)} u, \phi\right\rangle= & -\beta \int_{\partial B_{R}} \operatorname{div}_{\tau}\left[\partial_{n} u \partial_{n} V_{n} \nabla_{\tau} V_{n}\right] \phi d \sigma+\beta \int_{\partial B_{R}} \Delta_{\tau}\left[V_{n} \partial_{n} u \nabla_{\tau} V_{n}\right] \phi d \sigma, \\
\left\langle D^{(2,3)} u, \phi\right\rangle= & 2 \beta \int_{\partial B_{R}} \operatorname{div}_{\tau}\left[V_{n} D^{2} b \cdot \nabla_{\tau}\left[V_{n} \partial_{n} u\right]\right] \phi d \sigma .
\end{aligned}
$$

We denote by $D^{(1)}$ and $D^{(2, k)}, k=1,2,3$, the matrices whose elements are defined by

$$
D_{i j}^{(1)}=\left\langle D^{(1)} \tilde{u}_{i}, u_{j}\right\rangle, \quad \text { and } \quad D_{i j}^{(2, k)}=\left\langle D^{(2, k)} u_{i}, u_{j}\right\rangle, \quad i, j=1,2, \ldots, d .
$$


We give a result concerning the traces of the matrices.

Lemma C.1. We have

$$
\operatorname{Tr}\left(D^{(2,1)}\right)=\operatorname{Tr}\left(D^{(2,2)}\right)=0 \quad \text { and } \operatorname{Tr}\left(D^{(2,3)}\right)=-\frac{2 \beta(d-1) K(R)}{R} \int_{\partial B_{R}} V_{n}^{2} d \sigma,
$$

with the normalization constant $K(R)=\frac{d}{R^{2+d} \omega_{d}}$.

Proof. We have

$$
\begin{aligned}
\operatorname{Tr}\left(D^{(2,1)}\right)= & \beta\left[\int_{\partial B_{R}}-\Delta_{\tau}\left(W \sum_{i=1}^{d} \partial_{n} u_{i}\right) u_{i} d \sigma-\int_{\partial B_{R}} \operatorname{div}_{\tau}\left(V_{n} \partial_{n} V_{n} \sum_{i=1}^{d} \nabla_{\tau}\left(\partial_{n} u_{i}\right)\right) u_{i} d \sigma\right. \\
& \left.-\int_{\partial B_{R}} \sum_{i=1}^{d} \operatorname{div}_{\tau}\left(H V_{n} \nabla_{\tau}\left(V_{n} \partial_{n} u_{i}\right)\right) u_{i} d \sigma\right] \\
= & \beta \int_{\partial B_{R}} V_{n}^{\prime}(d-1) \sum_{i=1}^{d}\left|\partial_{n} u_{i}\right|^{2} \frac{d \sigma}{R}+\beta \int_{\partial B_{R}} V_{n} \partial_{n} V_{n} \sum_{i=1}^{d} \nabla_{\tau}\left(\partial_{n} u_{i}\right) \cdot \nabla_{\tau} u_{i} \partial \sigma \\
& +\beta \int_{\partial B_{R}} H V_{n}^{2} \sum_{i=1}^{d} \nabla_{\tau}\left(\partial_{n} u_{i}\right) \cdot \nabla_{\tau} u_{i} d \sigma+\beta \int_{\partial B_{R}} H \sum_{i=1}^{d} \partial_{n} u_{i} V_{n} \nabla_{\tau} V_{n} \cdot \nabla_{\tau} u_{i} \cdot d \sigma
\end{aligned}
$$

Combining the two facts (coming from algebraic properties of spherical harmonics, see Appendix B),

$$
(d-1) \sum_{i=1}^{d} \frac{\left|\partial_{n} u_{i}\right|^{2}}{R}=\sum_{i=1}^{d} \nabla_{\tau}\left(\partial_{n} u_{i}\right) \cdot \nabla_{\tau} u_{i}=\frac{d(d-1)}{R^{2+d} \omega_{d}}=(d-1) K(R)
$$

and

$$
\int_{\partial B_{R}} V_{n} \sum_{i=1}^{d} \partial_{n} u_{i} \nabla_{\tau} V_{n} \cdot \nabla_{\tau} u_{i}=0
$$

we get

$$
\operatorname{Tr}\left(D^{(2,1)}\right)=(d-1) \sum_{i=1}^{d}\left|\partial_{n} u_{i}\right|^{2} \int_{\partial B_{R}}\left(W+V_{n} \partial_{n} V_{n}+H V_{n}^{2}\right) \frac{d \sigma}{R} .
$$

Since we assumed the deformation to be volume preserving up to the second order $(41)$, we have $\operatorname{Tr}\left(D^{(2,1)}\right)=0$. The same strategy applies for $\operatorname{Tr}\left(D^{(2,2)}\right)$.

We focus now on $\operatorname{Tr}\left(D^{(2,3)}\right)$. We first expand the second term in the definition of $D^{(4)}$ :

$$
\begin{aligned}
\operatorname{Tr}\left(D^{(2,3)}\right)= & \beta \sum_{i=1}^{d} \int_{\partial B_{R}} V_{n} \partial_{n} u_{i} \nabla_{\tau}\left[\partial_{n} V_{n}\right] . \nabla_{\tau} u_{i}-2 V_{n} \partial_{n} u_{i} D^{2} b \nabla_{\tau} V_{n} \cdot \nabla_{\tau} u_{i} d \sigma \\
& -\beta \sum_{i=1}^{d} \int_{\partial B_{R}} 2 V_{n}^{2} D^{2} b \nabla_{\tau}\left(\partial_{n} u_{i}\right) \cdot \nabla_{\tau} u_{i} d \sigma .
\end{aligned}
$$

We follow the same argument thanks to the relations (55)-(56) and the fact

$$
\sum_{i=1}^{d} D^{2} b \nabla_{\tau}\left(\partial_{n} u_{i}\right) \cdot \nabla_{\tau} u_{i}=\frac{(d-1) K(R)}{R}
$$

on the sphere. Recall that on the sphere $D^{2} b=I_{d} / R$ when restricted to the tangent space. 
Study of $B\left(u, u^{\prime}, \phi\right)$. In the same manner, we begin to compute the derivative of the integrand:

$$
\left.\frac{d}{d t}\left(\mathcal{A}(t) V_{n} \nabla_{\tau} u . \nabla_{\tau} \phi\right)\right|_{t=0}=\mathcal{A}^{\prime} V_{n} \nabla_{\tau} u \cdot \nabla_{\tau} \phi+\mathcal{A} V_{n}^{\prime} \nabla_{\tau} u . \nabla_{\tau} \phi+\mathcal{A} V_{n} \nabla_{\tau} u^{\prime} \cdot \nabla_{\tau} \phi-\mathcal{A} V_{n} \partial_{n} u \nabla_{\tau} V_{n} . \nabla_{\tau} \phi .
$$

Denote $\mathcal{A}=\left(a_{i j}\right)_{1 \leq i, j \leq d}$ and $\tilde{\mathcal{A}}=\left(\partial_{n} a_{i j}\right)_{1 \leq i, j \leq d}$. Thanks to Lemma A.3, we get

$$
V_{n} \partial_{n}\left(V_{n} \mathcal{A} \cdot \nabla_{\tau} u \cdot \nabla_{\tau} \phi\right)=V_{n}^{2} \tilde{\mathcal{A}} \cdot \nabla_{\tau} u \cdot \nabla_{\tau} \phi+V_{n} \partial_{n} V_{n} \mathcal{A} \cdot \nabla_{\tau} u \cdot \nabla_{\tau} \phi+V_{n}^{2} \mathcal{A} \partial_{n}\left[\nabla_{\tau} u \cdot \nabla_{\tau} \phi\right] .
$$

From the relation

$$
\begin{aligned}
\beta \frac{d}{d t} \int_{\partial \Omega_{t}} \mathcal{A}(t) V_{n} \nabla_{\partial \Omega_{t}} u .\left.\nabla_{\partial \Omega_{t}} \phi d \sigma_{t}\right|_{t=0}= & \left.\int_{\partial B_{R}} \frac{d}{d t}\left(\mathcal{A}(t) V_{n} \nabla_{\partial \Omega_{t}} u \cdot \nabla_{\partial \Omega_{t}} \phi\right)\right|_{t=0} d \sigma \\
& +\int_{\partial B_{R}} V_{n} \partial_{n}\left(\mathcal{A} V_{n} \nabla_{\tau} u . \nabla_{\tau} \phi\right) d \sigma+\int_{\partial B_{R}} H V_{n}^{2} \mathcal{A} \nabla_{t} u . \nabla_{\tau} \phi d \sigma_{t},
\end{aligned}
$$

we gather all the terms and obtain $B(u, \phi)=\left\langle B^{(1)} u^{\prime}, \phi\right\rangle+\left\langle B^{(2)} u^{\prime}, \phi\right\rangle$; we then set

$$
\left\langle B^{(2)} u, \phi\right\rangle=\sum_{i=1}^{4}\left\langle B^{(2, i)} u, \phi\right\rangle
$$

where

$$
\begin{aligned}
& \left\langle B^{(2,1)} u^{\prime}, \phi\right\rangle=-\beta \int_{\partial B_{R}} \operatorname{div}_{\tau}\left[V_{n} A \cdot \nabla_{\tau} u^{\prime}\right] \phi d \sigma \\
& \left\langle B^{(2,1)} u, \phi\right\rangle=-\beta \int_{\partial B_{R}} \operatorname{div}_{\tau}\left[\left(W+H V_{n}^{2}+V_{n} \partial_{n} V_{n}\right) \mathcal{A} \cdot \nabla_{\tau} u\right] \phi d \sigma, \\
& \left\langle B^{(2,2)} u, \phi\right\rangle=-\beta \int_{\partial B_{R}} \operatorname{div}_{\tau}\left[\partial_{n} u V_{n} \mathcal{A} \cdot \nabla_{\tau} V_{n}\right] \phi d \sigma, \\
& \left\langle B^{(2,3)} u, \phi\right\rangle=-\beta \int_{\partial B_{R}} \operatorname{div}_{\tau}\left[V_{n} \mathcal{A}^{\prime} \cdot \nabla_{\tau} u\right] \phi d \sigma \\
& \left\langle B^{(2,4)} u, \phi\right\rangle=\beta \int_{\partial B_{R}} V_{n}^{2} \partial_{n}\left[\mathcal{A} \cdot \nabla_{\tau} u \cdot \nabla_{\tau} \phi\right] d \sigma .
\end{aligned}
$$

We get

$$
\begin{aligned}
\left\langle B^{(2,4)} u, \phi\right\rangle & =\beta \int_{\partial B_{R}} V_{n}^{2}\left(\partial_{n}[\mathcal{A}] \cdot \nabla_{\tau} u \cdot \nabla_{\tau} \phi\right) d \sigma+\beta \int_{\partial B_{R}} V_{n}^{2} \mathcal{A} \cdot \nabla_{\tau} \partial_{n} u \cdot \nabla_{\tau} \phi d \sigma-\beta \int_{\partial \Omega} 2\left(D^{2} b \mathcal{A}\right) \cdot \nabla_{\tau} u \cdot \nabla_{\tau} \phi d \sigma \\
& \left.=-\beta \int_{\partial B_{R}} \operatorname{div}_{\tau}\left[V_{n}^{2}\left(\tilde{\mathcal{A}} \cdot \nabla_{\tau} u+\mathcal{A} \cdot \nabla_{\tau}\left[\partial_{n} u\right]\right)-2 D^{2} b \mathcal{A} \cdot \nabla_{\tau} u\right)\right] \phi d \sigma
\end{aligned}
$$

Let $B^{(2, k)}, k=1,2,3,4$, denote the respective matrices associated to the operator with respect to the basis of eigenvectors. We have the following result:

Lemma C.2. We have

$$
\operatorname{Tr}\left(\sum_{i=1}^{4} B^{(2, i)}\right)=-\beta(d-1) R K(R) \int_{\partial B_{R}}\left|\nabla_{\tau} V_{n}\right|^{2} d \sigma+2 \frac{\beta K(R)}{R} \int_{\partial B_{R}} V_{n}^{2} d \sigma .
$$


Proof. Using the same arguments as before, we prove easily that $\operatorname{Tr}\left(B^{(2,1)}\right)=\operatorname{Tr}\left(B^{(2,2)}\right)=0$.

For the other terms, above all we have to focus on the term

$$
\operatorname{Tr}\left(B^{(2,3)}\right)=\beta \int_{\partial B_{R}} V_{n} \sum_{i=1}^{d}\left(\mathcal{A}^{\prime} \cdot \nabla_{\tau} u_{i}\right) \cdot \nabla_{\tau} u_{i} d \sigma .
$$

We have, thanks to the expression of shape derivation of the normal vector and of the mean curvature given in Proposition A.2:

$$
\mathcal{A}(t)=H(t)-2 D^{2} b(t) \quad \Rightarrow \quad \mathcal{A}^{\prime}=-\Delta_{\tau} V_{n}+2 D\left(\nabla_{\tau} V_{n}\right) ;
$$

then

$$
\begin{aligned}
\operatorname{Tr}\left(B^{(2,3))}\right. & =\beta \int_{\partial B_{R}} V_{n} \sum_{i=1}^{d}\left(\mathcal{A}^{\prime} \cdot \nabla_{\tau} u_{i}\right) \cdot \nabla_{\tau} u_{i} d \sigma \\
& =-\beta \int_{\partial B_{R}} V_{n} \Delta_{\tau} V_{n} \sum_{i=1}^{d}\left|\nabla_{\tau} u_{i}\right|^{2} d \sigma+2 \beta \int_{\partial B_{R}} V_{n} \sum_{i=1}^{d}\left[D\left(\nabla_{\tau} V_{n}\right) \cdot \nabla_{\tau} u_{i}\right] \cdot \nabla_{\tau} u_{i} d \sigma \\
& =-\beta \int_{\partial B_{R}} V_{n} \Delta_{\tau} V_{n} \sum_{i=1}^{d}\left|\nabla_{\tau} u_{i}\right|^{2} d \sigma+2 \beta \int_{\partial B_{R}} V_{n} \sum_{i=1}^{d}\left[D_{\tau}\left(\nabla_{\tau} V_{n}\right) \cdot \nabla_{\tau} u_{i}\right] \cdot \nabla_{\tau} u_{i} d \sigma \\
& =-\beta \int_{\partial B_{R}} V_{n} \Delta_{\tau} V_{n} \sum_{i=1}^{d}\left|\nabla_{\tau} u_{i}\right|^{2} d \sigma+2 \beta \int_{\partial B_{R}} V_{n} \sum_{i=1}^{d}\left[D_{\tau}^{2} V_{n} \cdot \nabla_{\tau} u_{i}\right] \cdot \nabla_{\tau} u_{i} d \sigma \\
& =-\beta \int_{\partial B_{R}} V_{n} \Delta_{\tau} V_{n} \sum_{i=1}^{d}\left|\nabla_{\tau} u_{i}\right|^{2} d \sigma+2 \beta \int_{\partial B_{R}} V_{n} \operatorname{Tr}\left(D_{\tau}^{2} V_{n}\right) \sum_{i=1}^{d}\left|\nabla_{\tau} u_{i}\right|^{2} d \sigma
\end{aligned}
$$

Since $\operatorname{Tr}\left(D_{\tau}^{2} V_{n}\right)=\Delta_{\tau} V_{n}$, and since $\sum_{i=1}^{d}\left|\nabla_{\tau} u_{i}\right|^{2}=R K(R)$, on $\partial B_{R}$ we get

$$
\operatorname{Tr}\left(B^{(2,3)}\right)=\beta \int_{\partial B_{R}} V_{n} \Delta_{\tau} V_{n} \sum_{i=1}^{d}\left|\nabla_{\tau} u_{i}\right|^{2} d \sigma=\beta(d-1) R K(R) \int_{\partial B_{R}} V_{n} \Delta_{\tau} V_{n} d \sigma .
$$

Concerning $\operatorname{Tr}\left(B^{(2,4)}\right)$, we have to distinguish the case $d=2$ from the case $d=3$. If $d=3$ then $\mathcal{A}=0$; this implies that $\operatorname{Tr}\left(B^{(2,4)}\right)$ is reduced to

$$
\operatorname{Tr}\left(B^{(2,4)}\right)=(d-1) K(R) \frac{\beta}{R} \int_{\partial B_{R}} V_{n}^{2} d \sigma .
$$

If $d=2$, then $\mathcal{A}+\tilde{\mathcal{A}}$ is a null matrix and this leads to

$$
\begin{aligned}
\operatorname{Tr}\left(B^{(2,4)}\right) & =2 \beta \int_{\partial B_{R}} V_{n}^{2} \sum_{i=1}^{d} D^{2} b \cdot \nabla_{\tau} u_{i} \cdot \nabla_{\tau} u_{i} d \sigma \\
& =2 K(R) \frac{\beta}{R} \int_{\partial B_{R}} V_{n}^{2} d \sigma .
\end{aligned}
$$

Then for $d=2,3$ we get

$$
\operatorname{Tr}\left(B^{(2,4)}\right)=2 \beta \frac{K(R)}{R} .
$$


Study of $A\left(u, u^{\prime}, \phi\right)$. We have

$$
\begin{aligned}
\left.\frac{d}{d t}\left(\int_{\partial \Omega_{t}} V_{n} \nabla_{\tau} u \cdot \nabla_{\tau} \phi d \sigma_{t}\right)\right|_{t=0}= & \int_{\partial B_{R}} W \nabla_{\tau} u \cdot \nabla_{\tau} \phi d \sigma+\int_{\partial B_{R}} V_{n} \nabla_{\tau} u^{\prime} \cdot \nabla_{\tau} \phi d \sigma \\
& +\int_{\partial B_{R}} V_{n} \nabla_{\tau} V_{n} \cdot\left[\partial_{n} u \nabla_{\tau} \phi+\partial_{n} \phi \nabla_{\tau} u\right] \\
& +\left(V_{n} \partial_{n}\left[V_{n} \nabla_{\tau} u \cdot \nabla_{\tau} \phi\right]+H V_{n}^{2} \nabla_{\tau} u \cdot \nabla_{\tau} \phi\right) d \sigma .
\end{aligned}
$$

Since $\partial_{n} \phi=0$, it comes that

$$
\begin{aligned}
\int_{\partial B_{R}} V_{n} \nabla_{\tau} V_{n} \cdot\left[\partial_{n} u \nabla_{\tau} \phi+\partial_{n} \phi \nabla_{\tau} u\right] d \sigma= & -\frac{1}{2} \int_{\partial B_{R}} V_{n}^{2}\left[\partial_{n} u \Delta_{\tau} \phi+\nabla_{\tau}\left[\partial_{n} u\right] . \nabla_{\tau} \phi\right] \\
= & -\frac{1}{2} \int_{\partial B_{R}} V_{n}^{2}\left(\partial_{n}\left[\nabla_{\tau} u \cdot \nabla_{\tau} \phi\right]+2 D^{2} b \nabla_{\tau} u \cdot \nabla_{\tau} \phi\right) d \sigma \\
& -\frac{1}{2} \int_{\partial B_{R}} V_{n}^{2} \partial_{n} u \Delta_{\tau} \phi d \sigma
\end{aligned}
$$

Hence, gathering the equivalent terms we get

$$
\begin{aligned}
\left.\frac{d}{d t} \int_{\partial \Omega_{t}} V_{n} \nabla_{\tau} u \cdot \nabla_{\tau} \phi d \sigma_{t}\right|_{t=0}= & \int_{\partial B_{R}} W \nabla_{\tau} u^{\prime} . \nabla_{\tau} \phi d \sigma+\int_{\partial B_{R}} V_{n} \nabla_{\tau} u^{\prime} . \nabla_{\tau} \phi d \sigma \\
& -\frac{1}{2} \int_{\partial B_{R}} \Delta_{\tau}\left[V_{n}^{2} \partial_{n} u\right] \phi-\partial_{n}\left(V_{n}^{2} \nabla_{\tau} u \cdot \nabla_{\tau} \phi\right) d \sigma \\
& +\int_{\partial B_{R}}\left(H I_{d}-D^{2} b\right) V_{n}^{2} \nabla_{\tau} u \cdot \nabla_{\tau} \phi d \sigma .
\end{aligned}
$$

We split these terms into $A(u, \phi)=\left\langle A^{(1)} u^{\prime}, \phi\right\rangle+\left\langle A^{(2)} u, \phi\right\rangle$. As before, we set $\left\langle A^{(2)} u, \phi\right\rangle=\sum_{i=1}^{3}\left\langle A^{(i)} u, \phi\right\rangle$ where

$$
\begin{aligned}
& \left\langle A^{(1)} u^{\prime}, \phi\right\rangle=\int_{\partial B_{R}}-\operatorname{div}_{\tau}\left[V_{n} \nabla_{\tau} u^{\prime}\right] \phi d \sigma, \\
& \left\langle A^{(2,1)} u, \phi\right\rangle=\int_{\partial B_{R}}-\operatorname{div}_{\tau}\left[\left(W+H V_{n}^{2}+V_{n} \partial_{n} V_{n}\right) \nabla_{\tau} u\right] \phi d \sigma, \\
& \left\langle A^{(2,2)} u, \phi\right\rangle=\int_{\partial B_{R}} \operatorname{div}_{\tau}\left[\partial_{n} u V_{n} \nabla_{\tau} V_{n}\right] \phi d \sigma, \\
& \left\langle A^{(2,3)} u, \phi\right\rangle=\int_{\partial B_{R}} \operatorname{div}_{\tau}\left[V_{n}^{2}\left(2 D^{2} b \nabla_{\tau} u-\nabla_{\tau}\left(\partial_{n} u\right)\right)\right] \phi d \sigma .
\end{aligned}
$$

We have

Lemma C.3. We have

$$
\operatorname{Tr}\left(A^{(2,1)}\right)=0, \quad \operatorname{Tr}\left(A^{(2,2)}\right)=0 \quad \text { and } \quad \operatorname{Tr}\left(A^{(2,3)}\right)=-K(R) \int_{\partial B_{R}} V_{n}^{2} d \sigma .
$$


The proof of Lemma C.3 follows the lines of the proof of Lemma C.2.

Study of $C\left(u, u^{\prime}, u^{\prime \prime}, \phi\right)$. We decompose $C\left(u, u^{\prime}, u^{\prime \prime}, \phi\right)$ as follows:

$$
C(u, \phi)=\left\langle C^{(0)} u^{\prime \prime}, \phi\right\rangle+\left\langle C^{(1)} u^{\prime}, \phi\right\rangle+\left\langle C^{(2)} u, \phi\right\rangle
$$

with $\left\langle C^{(2)} u, \phi\right\rangle=\sum_{i=3}^{6}\left\langle C^{(2, i)} u, \phi\right\rangle$ where

$$
\begin{aligned}
\left\langle C^{(0)} u^{\prime \prime}, \phi\right\rangle & =-\lambda \int_{\partial B_{R}} u^{\prime \prime} \phi d \sigma \\
\left\langle C^{(1)} u^{\prime}, \phi\right\rangle & =-2 \int_{\partial B_{R}}\left(\lambda^{\prime} u^{\prime}+\lambda V_{n}\left(\partial_{n} u^{\prime}+H u^{\prime}\right)\right) \phi d \sigma \\
\left\langle C^{(2,1)} u, \phi\right\rangle & =-\lambda^{\prime \prime} \int_{\partial B_{R}} u \phi-\lambda^{\prime} \int_{\partial B_{R}} V_{n} \partial_{n} u \phi d \sigma \\
\left\langle C^{(2,2)} u, \phi\right\rangle & =-\lambda \int_{\partial B_{R}}\left(W+V_{n} \partial_{n} V_{n}+H V_{n}^{2}\right)\left(\partial_{n} u+H u\right) \phi d \sigma \\
\left\langle C^{(2,3)} u, \phi\right\rangle & =-\lambda \int_{\partial B_{R}} V_{n}\left(-\nabla_{\tau} V_{n} \cdot \nabla_{\tau} u+H^{\prime} u\right) \phi d \sigma \\
& =\lambda \int_{\partial B_{R}} V_{n}\left(\nabla_{\tau} V_{n} \cdot \nabla_{\tau} u+\Delta_{\tau} V_{n} u\right) \phi d \sigma \\
\left\langle C^{(2,4)} u, \phi\right\rangle & =-\lambda \int_{\partial B_{R}} V_{n}^{2}\left(\partial_{n}^{2} u-u \sum_{i=1}^{d-1} \kappa_{i}^{2}+H \partial_{n} u\right) \phi d \sigma \\
& =0 .
\end{aligned}
$$

Denoting by $\left(C^{(2, j)}\right), j=1,2,3,4$, the matrices associated to the linear operators $C^{(2, p)}, p=1,2,3,4$, in the basis of eigenvectors, we get:

Lemma C.4. We have

$$
\sum_{j=1}^{4} \operatorname{Tr}\left(C^{(2, j)}\right)=\lambda R^{3} K(R) \int_{\partial B_{R}} V_{n} \Delta_{\tau} V_{n} d \sigma=-((d-1) \beta+R) R K(R) \int_{\partial B_{R}}\left|\nabla_{\tau} V_{n}\right|^{2} d \sigma .
$$

Proof. The proof is straightforward and obeys to the same arguments used before. The only nonnull trace concerns the factor in $-H^{\prime}=\Delta_{\tau} V_{n}$.

\section{Appendix D. Computing $u^{\prime}$}

In this section, we focus on the computation of the trace of $E^{(1)}$ introduced in Section 4.1. We recall that $t \mapsto$ $(\lambda(t), u(t, \cdot))$ is solution of

$$
\begin{array}{ll}
\Delta u=0 & \text { in } T_{t}\left(B_{R}\right), \\
-\beta \Delta_{\tau} u+\partial_{n} u-\lambda(t) u=0 & \text { on } \partial T_{t}\left(B_{R}\right) .
\end{array}
$$

To compute the second derivative, one must know $u^{\prime}=u^{\prime}(0)$. For the reader convenience, we recall the problem (36) solved by $u^{\prime}$. 


$$
\begin{aligned}
\Delta u^{\prime}=0 \quad \text { in } B_{R}, \\
\begin{aligned}
-\beta \Delta_{\tau} u^{\prime}+\partial_{n} u^{\prime}-\lambda v^{\prime}= & \beta \Delta_{\tau}\left(V_{n} \partial_{n} u\right)-\beta \operatorname{div}_{\tau}\left(V_{n}\left(2 D^{2} b-H I_{d}\right) \nabla_{\tau} u\right) \\
& +\operatorname{div}_{\tau}\left(V_{n} \nabla_{\tau} u\right)-\lambda^{\prime} u+\lambda V_{n}\left(\partial_{n} u+H u\right) \quad \text { on } \partial B_{R} .
\end{aligned}
\end{aligned}
$$

First, Fredholm's alternative insures the existence of a unique harmonic function $\tilde{u}_{j}$ orthogonal to the eigenfunctions $u_{1}, u_{2}, \ldots, u_{d}$ and satisfying on $\partial B_{R}$ the boundary condition

$$
\begin{aligned}
-\beta \Delta_{\tau} \tilde{u}_{j}+\partial_{n} \tilde{u}_{j}-\lambda \tilde{u}_{j}= & \beta\left[\Delta_{\tau}\left[V_{n} \partial_{n} u_{j}\right]+\operatorname{div}_{\tau}\left[V_{n}\left(H I_{d}-2 D^{2} b\right) \cdot \nabla_{\tau} u_{j}\right]\right] \\
& +\operatorname{div}_{\tau}\left[V_{n} \nabla_{\tau} u_{j}\right]+\lambda^{\prime} u_{j}+\lambda V_{n}\left(\partial_{n} u_{j}+H u_{j}\right) .
\end{aligned}
$$

It follows that

$$
u^{\prime}=\sum_{j=1}^{d} \tilde{c}_{j} u_{j}+\sum_{j=1}^{m} c_{j} \tilde{u}_{j}
$$

for some $c_{j}, \tilde{c}_{j}$ when $j=1, \ldots, d$. We point out that the $\left(c_{j}\right)$ are the same coefficients as the decomposition of $u$ in the basis $\left(u_{j}\right)$ of the eigenspace associated to $\lambda: u=c_{1} u_{1}+\cdots+c_{d} u_{d}$.

Remark D.1. We recall that we only need the terms $\tilde{u}_{j}$ : we inject this decomposition of $u^{\prime}$ in $E^{(1)}$ :

$$
\begin{aligned}
E^{(1)} \phi= & -2 \sum_{j=1}^{d} \tilde{c}_{j}\left[\int_{\partial B_{R}} V_{n} \partial_{n} u_{j} \partial_{n} \phi d \sigma+2 \frac{R+\beta(d-3)}{R} \int_{\partial B_{R}} V_{n} \nabla_{\tau} u_{j} . \nabla_{\tau} \phi d \sigma\right] \\
& -2 \sum_{j=1}^{m} c_{j}\left[\int_{\partial B_{R}} V_{n} \partial_{n} \tilde{u}_{j} \partial_{n} \phi d \sigma+2 \frac{R+\beta(d-3)}{R} \int_{\partial B_{R}} V_{n} \nabla_{\tau} \tilde{u}_{j} . \nabla_{\tau} \phi d \sigma\right. \\
& \left.-2 \lambda \int_{\partial B_{R}} V_{n} H u_{j} \phi d \sigma-2 \lambda \int_{\partial B_{R}} V_{n} H \tilde{u}_{j} \phi d \sigma\right] .
\end{aligned}
$$

By construction the first sum cancels and we simply get

$$
E_{j k}^{(1)}=2 \int_{\partial \Omega} V_{n}\left(-\partial_{n} \tilde{u}_{j} \partial_{n} u_{k}-H \lambda \tilde{u}_{j} u_{k}+\left(I+\beta\left(H I_{d}-2 D^{2} b\right)\right) \nabla_{\tau} \tilde{u}_{j} . \nabla_{\tau} u_{k}\right) d \sigma
$$

\section{D.1. Explicit resolution of (58) to compute $\tilde{u}_{j}$}

Let us now compute $\tilde{u}_{j}$ solution of (58). This step consists in technical computations. For the completeness of the presentation, we present the case of dimension three, we will then simply state the results in dimension two. From now on, we do not consider the case $d \geq 4$ for technical reasons.

\section{D.1.1. Explicit representation of $\tilde{u}_{j}$ in the case $d=2$}

We illustrate the computation of the elements $\tilde{u}_{i}, i=1,2$ in the case $d=2$. The eigenfunctions are the normalized coordinate functions that is $\left(u_{1}, u_{2}\right)$ given by

We have

$$
u_{1}(r, \theta)=r \frac{\cos \theta}{\sqrt{\pi R^{3}}} \quad \text { and } \quad u_{2}(r, \theta)=r \frac{\sin \theta}{\sqrt{\pi R^{3}}} .
$$

Lemma D.2. Let $V$ be a deformation of normal component $V_{n}=R^{k}\left(v_{1}^{(k)} \cos k \theta+v_{2}^{(k)} \sin k \theta\right)$, then

$$
\begin{aligned}
\tilde{u}_{1}(r, \theta)= & \frac{r^{k+1}}{2 \sqrt{\pi} R^{\frac{7}{2}}} \frac{1-k}{k}\left[v_{1}^{(k)} \cos (k+1) \theta+v_{2}^{k} \sin (k+1) \theta\right] \\
& +\frac{r^{k-1}}{2 \sqrt{\pi} R^{\frac{3}{2}}} \frac{1+k-2}{k-2}\left[\frac{\beta(2-k)+R}{k \beta+R}\right]\left[v_{1}^{(k)} \cos (k-1) \theta+v_{2}^{k} \sin (k-1) \theta\right]
\end{aligned}
$$


and

$$
\begin{aligned}
\tilde{u}_{2}(r, \theta)= & \frac{r^{k+1}}{2 \sqrt{\pi} R^{\frac{7}{2}}} \frac{1-k}{k}\left[-v_{2}^{(k)} \cos (k+1) \theta+v_{1}^{k} \sin (k+1) \theta\right] \\
& +\frac{r^{k-1}}{2 \sqrt{\pi} R^{\frac{3}{2}}} \frac{1+k}{k-2}\left[\frac{\beta(2-k)+R}{k \beta+R}\right]\left[v_{2}^{(k)} \cos (k-1) \theta-v_{1}^{k} \sin (k-1) \theta\right]
\end{aligned}
$$

In order to justify these formulae, one has to compute $a, b, c, d$ the coefficients

$$
\tilde{u}_{j}=a^{(k)} \cos (k+1) \theta+b^{(k)} \sin (k+1) \theta+c^{(k)} \cos (k-1) \theta+d^{(k)} \cos (k-1) \theta
$$

such that $\tilde{u}_{j}$ satisfies (58) with $u_{i}=\frac{x_{i}}{\left\|x_{i}\right\|_{L^{2}\left(\partial B_{R}\right)}}$. We left the tedious computations to the reader.

\section{D.1.2. Explicit representation of $\tilde{u}_{j}$ in the case $d=3$}

We begin with the case where $V_{n}=r^{l} Y_{l}^{m}$ and $\varphi_{p}=r Y_{1}^{p}$ where $-l \leq m \leq l$ and $-1 \leq p \leq 1$. We introduce the coefficients:

$$
C_{l-1, p}^{(l, 1, m, p)}=(-1)^{m+p} \sqrt{\frac{3(2 l-1)(2 l+1)}{4 \pi}}\left(\begin{array}{ccc}
l & 1 & l-1 \\
m & p & -m-p
\end{array}\right)\left(\begin{array}{ccc}
l & 1 & l-1 \\
0 & 0 & 0
\end{array}\right),
$$

and

$$
C_{l+1, p}^{(l, 1, m, p)}=(-1)^{m+p} \sqrt{\frac{3(2 l+1)(2 l+3)}{4 \pi}}\left(\begin{array}{ccc}
l & 1 & l+1 \\
m & p & -m-p
\end{array}\right)\left(\begin{array}{ccc}
l & 1 & l+1 \\
0 & 0 & 0
\end{array}\right),
$$

where we use the Wigner $3 j$ symbol and Clebsch-Gordon coefficients. We set $\alpha=\beta / R$ in order to obtain an adimensional constant.

Lemma D.3. Let $l \neq 0$ be a natural integer and let $-l \leq m \leq l$. Let $V_{n}=r^{l} Y_{l}^{m}$ and $u_{p}=r Y_{1}^{p}$ where $-1 \leq p \leq 1$. The unique solution of (58) that is orthogonal to $\operatorname{Span}\left(Y_{1}^{-1}, Y_{1}^{0}, Y_{1}^{1}\right)$ is given by

$$
\tilde{u}_{p}=a_{l-1, p, \alpha}^{(l, 1, m, p)} r^{l-1} Y_{l-1}^{m+p}+a_{l+1, p, \alpha}^{(l, 1, m, p)} \frac{r^{l+1}}{R^{2}} Y_{l+1}^{m+p}
$$

where

$$
a_{l-1, p, \alpha}^{(l, 1, m, p)}=\frac{l+2}{l-2} \frac{1+\alpha(3-l)}{1+\alpha(1+l)} C_{l-1, p}^{(l, 1, m, p)} \quad \text { and } \quad a_{l+1, p, \alpha}^{(l, 1, m, p)}=\frac{l-1}{l} \frac{1+\alpha(4+l)}{1+\alpha(3+l)} C_{l+1, p}^{(l, 1, m, p)} .
$$

Proof. We first decompose the right hand side of (58) into the basis of spherical harmonics. Taking into account that

$$
\left(\begin{array}{ccc}
l_{1} & l_{2} & L \\
0 & 0 & 0
\end{array}\right)=0
$$

whenever $\left(l_{1}, l_{2}, L\right)$ satisfies the triangular inequality and $l_{1}+l_{2}+L$ is odd, we get

$$
\beta V_{n} \partial_{n} u_{p}=\beta R^{l} Y_{l}^{m} Y_{1}^{p}=\beta R^{l}\left[C_{l-1, p}^{(l, 1, m, p)} Y_{l-1}^{m+p}+C_{l+1, p}^{(l, 1, m, p)} Y_{l+1}^{m+p}\right]
$$

and then

$$
\beta \Delta_{\tau}\left(V_{n} \partial_{n} u_{p}\right)=\alpha R^{l-1}\left[l(1-l) C_{l-1, p}^{(l, 1, m, p)} Y_{l-1}^{m+p}-(l+1)(l+2) C_{l+1, p}^{(l, 1, m, p)} Y_{l+1}^{m+p}\right] .
$$

We also have

$$
\begin{aligned}
\nabla_{\tau} V_{n} . \nabla_{\tau} u_{p}= & \frac{1}{2}\left[\Delta_{\tau}\left(V_{n} u_{p}\right)-V_{n} \Delta_{\tau} u_{p}-u_{p} \Delta_{\tau} V_{n}\right] \\
= & \frac{R^{l-1}}{2}\left[l(1-l) C_{l-1, p}^{(l, 1, m, p)} Y_{l-1}^{m+p}-(l+1)(l+2) C_{l+1, p}^{(l, 1, m, p)} Y_{l+1}^{m+p}\right. \\
& +2 C_{l-1, p}^{(l, 1, m, p)} Y_{l-1}^{m+p}+2 C_{l+1, p}^{(l, 1, m, p)} Y_{l+1}^{m+p} \\
& \left.+l(l+1) C_{l-1, p}^{(l, 1, m, p)} Y_{l-1}^{m+p}+l(l+1) C_{l+1, p}^{(l, 1, m, p)} Y_{l+1}^{m+p}\right] \\
= & R^{l-1}\left[(l+1) C_{l-1, p}^{(l, 1, m, p)} Y_{l-1}^{m+p}-l C_{l+1, p}^{(l, 1, m, p)} Y_{l+1}^{m+p}\right] .
\end{aligned}
$$


Since $\operatorname{div}_{\tau} V_{n} \nabla_{\tau} u_{p}=\nabla_{\tau} V_{n} \cdot \nabla_{\tau} u_{p}+V_{n} \Delta_{\tau} u_{p}$, it comes

$$
\operatorname{div}_{\tau} V_{n} \nabla_{\tau} u_{p}=R^{l-1}\left[(l-1) C_{l-1, p}^{(l, 1, m, p)} Y_{l-1}^{m+p}-(l+2) C_{l+1, p}^{(l, 1, m, p)} Y_{l+1}^{m+p}\right] .
$$

Hence, gathering the various terms on the right hand side of (58), we see that $\tilde{u}_{p}$ is solution of

$$
\begin{aligned}
& -\beta \Delta_{\tau} \tilde{u}_{p}+\partial_{n} \tilde{u}_{p}-\lambda_{2} \tilde{u}_{p} \\
& \quad=R^{l-1}\left[(l+2)(1+\alpha(3-l)) C_{l-1, p}^{(l, m, 1, p)} Y_{l-1}^{m+p}+(1-l)(1+\alpha(4+l)) C_{l+1, p}^{(l, m, 1, p)} Y_{l+1}^{m+p}\right] .
\end{aligned}
$$

After identification, we obtain:

$$
\tilde{u}_{p}=a_{l-1, p, \alpha}^{(l, 1, m, p)} r^{l-1} Y_{l-1}^{m+p}+a_{l+1, p, \alpha}^{(l, 1, m, p)} \frac{r^{l+1}}{R^{2}} Y_{l+1}^{m+p},
$$

where the coefficients $a_{l \pm 1, p, \alpha}^{(l, 1, m, p)}$ are defined in Lemma D.3.

As a corollary, we deduce the general case for $V_{n}$.

\section{Corollary D.4. If}

$$
V_{n}=\sum_{l=2}^{\infty} r^{l} \sum_{m=-l}^{l} v_{l, m} Y_{l}^{m} \quad \text { and } \quad u_{p}=\sum_{p=-1}^{1} \alpha_{p} Y_{1}^{p}
$$

then

$$
\tilde{u}_{p}=\sum_{l=2}^{\infty} \sum_{m=-l}^{l} \sum_{p=-1}^{1} \alpha_{p} v_{l, m}\left[a_{l-1, p, \alpha}^{(l, 1, m, p)} r^{l-1} Y_{l-1}^{m+p}+a_{l+1, p, \alpha}^{(l, 1, m, p)} \frac{r^{l+1}}{R^{2}} Y_{l+1}^{m+p}\right] .
$$

\section{D.2. The explicit expression of the trace of $E^{(1)}$}

We leave the tedious but easy computations of the case $d=2$ to the reader; the obtained result is written in (46). We focus here on the much more technical case $d=3$.

We set $u_{j}=K(R)\left(\alpha_{-1}^{i} Y_{1}^{-1}+\alpha_{0}^{i} Y_{1}^{0}+\alpha_{1}^{i} Y_{1}^{1}\right)$ for $1 \leq j \leq 3$ where

$$
\begin{array}{lll}
\alpha_{-1}^{1}=1 / \sqrt{2}, & \alpha_{0}^{1}=0, & \alpha_{1}^{1}=1 / \sqrt{2}, \\
\alpha_{-1}^{2}=0, & \alpha_{0}^{2}=1, & \alpha_{1}^{2}=0, \\
\alpha_{-1}^{3}=-i / \sqrt{2}, & \alpha_{0}^{3}=0, & \alpha_{1}^{3}=i / \sqrt{2} .
\end{array}
$$

On the sphere in dimension 3 , the deviatoric part of the curvature cancels and the entries of $E^{(1)}$ are

$$
\operatorname{Tr}\left(E^{(1)}\right)=\sum_{j=1}^{3} E_{j j}^{(1)} \quad \text { where } E_{j j}^{(1)}=\int_{\partial \Omega} V_{n}\left(-\partial_{n} \tilde{u}_{j} \partial_{n} u_{j}-H \lambda \tilde{u}_{j} u_{j}+\nabla_{\tau} \tilde{u}_{j} . \nabla_{\tau} u_{j}\right) d \sigma
$$

where each $\tilde{u}_{j}$ corresponding to $u_{j}$ is computed thanks to Corollary D.4.

We first state a technical result to perform this summation. We postpone its proof to the end of the section.

Lemma D.5. Let $V_{n}=R^{l} Y_{l}^{m},-l \leq m \leq l$ and

$$
\psi=r Y_{1}^{p}
$$

for $-1 \leq p \leq 1$. Let $m^{\prime}$ and $p^{\prime}$ be integers such that $-l \leq m^{\prime} \leq l$ and $-1 \leq p^{\prime} \leq 1$ and suppose

$$
\tilde{\psi}=a r^{l-1} Y_{l-1}^{m^{\prime}+p^{\prime}}+b \frac{r^{l+1}}{R^{2}} Y_{l+1}^{m^{\prime}+p^{\prime}} .
$$

Then 


$$
\begin{aligned}
& \int_{\partial B_{R}} V_{n}\left(-\partial_{n} \tilde{\psi} \partial_{n} \psi-H \lambda \tilde{\psi} \psi+\nabla_{\tau} \tilde{\psi} \cdot \nabla_{\tau} \psi\right) d \sigma \\
& \quad=-a(4 \alpha+2 l) R^{2 l-1} \int_{\partial B_{1}} Y_{l-1}^{m^{\prime}+p^{\prime}} Y_{l}^{m} Y_{1}^{p}-b(4 \alpha+2) R^{2 l-1} \int_{\partial B_{1}} Y_{l+1}^{m^{\prime}+p^{\prime}} Y_{l}^{m} Y_{1}^{p} .
\end{aligned}
$$

As a consequence, we get for $j=1,2,3$

$$
\begin{aligned}
E_{j j}^{(1)}= & -K(R) R^{2 l+1}\left[(4 \alpha+2 l) \frac{l+2}{l-2} \frac{1+\alpha(3+l)}{1+\alpha(1+l)} \sum_{m=-l}^{l} \sum_{p=-1}^{1}\left|\alpha_{p}^{j}\right|^{2}\left|v_{l, m}\right|^{2}\left(\int_{\partial B_{1}} \overline{Y_{l-1}^{m+p}} Y_{l}^{m} Y_{1}^{p}\right)^{2}\right. \\
& \left.+(4 \alpha+2) \frac{l-1}{l} \frac{1+\alpha(4+l)}{1+\alpha(3+l)} \sum_{m=-l}^{l} \sum_{p=-1}^{1}\left|\alpha_{p}^{j}\right|^{2}\left|v_{l, m}\right|^{2}\left(\int_{\partial B_{1}} \overline{Y_{l+1}^{m+p}} Y_{l}^{m} Y_{1}^{p}\right)^{2}\right] .
\end{aligned}
$$

We are now in a position to prove Proposition 4.3 concerning the trace of $E^{(1)}$ in dimension $d=3$.

Proof of Proposition 4.3. We have to sum the $E_{j j}^{(1)}$ obtained before the statement of Proposition 4.3. By the normalization condition $\sum_{j}\left|\alpha_{p}^{j}\right|^{2}=1$, our main task is to compute the sum over $p=-1,0,1$ of the integrals involving three spherical harmonics. The values of this type of integral is recalled in Propositions B.2 and B.3. Elementary computations then give

$$
\sum_{m=-l}^{l} \sum_{p=-1}^{1}\left(\int_{\partial B_{1}} \overline{Y_{l-1}^{m+p}} Y_{l}^{m} Y_{1}^{p}\right)^{2}=\frac{3}{4 \pi} \frac{l}{2 l+1} \text { and } \sum_{m=-l}^{l} \sum_{p=-1}^{1}\left(\int_{\partial B_{1}} \overline{Y_{l+1}^{m+p}} Y_{l}^{m} Y_{1}^{p}\right)^{2}=\frac{3}{4 \pi} \frac{l+1}{2 l+1} .
$$

Proof of Lemma D.5. We compute:

$$
\begin{aligned}
& -V_{n} \partial_{n} \tilde{\psi} \partial_{n} \psi=-R^{2 l-1}\left[a(l-1) Y_{l-1}^{m^{\prime}+p^{\prime}}+b(l+1) Y_{l+1}^{m^{\prime}+p^{\prime}}\right] Y_{l}^{m} Y_{1}^{p}, \\
& -\lambda H V_{n} \tilde{\psi} \psi=-R^{2 l-1}(4 \alpha+2)\left[a Y_{l-1}^{m^{\prime}+p^{\prime}}+b Y_{l+1}^{m^{\prime}+p^{\prime}}\right] Y_{l}^{m} Y_{1}^{p} .
\end{aligned}
$$

We have also

$$
\begin{aligned}
\int_{\partial B_{R}} V_{n} \nabla_{\tau} \tilde{\psi} \cdot \nabla_{\tau} \psi= & \frac{1}{2} \int_{\partial B_{R}} V_{n}\left[\Delta_{\tau}(\tilde{\psi} \psi)-\psi \Delta_{\tau} \tilde{\psi}-\tilde{\psi} \Delta_{\tau} \psi\right] \\
= & -\frac{1}{2} l(l+1) R^{2 l-1} \int_{\partial B_{1}}\left(a Y_{l-1}^{m^{\prime}+p^{\prime}}+b Y_{l+1}^{m^{\prime}+p^{\prime}}\right) Y_{l}^{m} Y_{1}^{p}+R^{2 l+1} \int_{\partial B_{1}}\left(a Y_{l-1}^{m^{\prime}+p^{\prime}}+b Y_{l+1}^{m^{\prime}+p^{\prime}}\right) Y_{l}^{m} Y_{1}^{p} \\
& +\frac{1}{2} R^{2 l-1} \int_{\partial B_{1}}\left[a l(l-1) Y_{l-1}^{m^{\prime}+p^{\prime}}+b(l+1)(l+2) Y_{l+1}^{m^{\prime}+p^{\prime}}\right] Y_{l}^{m} Y_{1}^{p} \\
= & R^{2 l-1} \int_{\partial B_{1}}\left[a(l-1) Y_{l-1}^{m^{\prime}+p^{\prime}}+b(l+2) Y_{l+1}^{m^{\prime}+p^{\prime}}\right] Y_{l}^{m} Y_{1}^{p} .
\end{aligned}
$$

We obtain the result by summing the three terms.

\section{Appendix E. Shape Derivatives of Steklov and Laplace-Beltrami eigenvalues problem}

The following result is obtained by taking $\beta=0$ in Theorem 1.4.

Theorem E.1 (Steklov eigenvalues). We distinguish the case of simple and multiple eigenvalue. 
- If $\lambda=\lambda_{k}(\Omega)$ is a simple eigenvalue of the Steklov problem and $u$ an associated eigenfunction, then the application $t \rightarrow \lambda(t)=\lambda_{k}((I+t \boldsymbol{V})(\Omega))$ is differentiable and the derivative at $t=0$ is

$$
\lambda^{\prime}(0)=\int_{\partial \Omega} V_{n}\left(\left|\nabla_{\tau} u\right|^{2}-\left|\partial_{n} u\right|^{2}-\lambda H|u|^{2}\right) d \sigma .
$$

The shape derivative $u^{\prime}$ of the eigenfunction satisfies

$$
\begin{aligned}
& \Delta u^{\prime}=0 \quad \text { in } \Omega, \\
& \partial_{n} u^{\prime}-\lambda u^{\prime}=\operatorname{div}_{\tau}\left(V_{n} \nabla_{\tau} u\right)-\lambda^{\prime}(0) u+\lambda V_{n}\left(\partial_{n} u+H u\right) \quad \text { on } \partial \Omega .
\end{aligned}
$$

- Let $\lambda$ be a multiple eigenvalue of order $m \geq 2$. Let $\left(u_{j}\right)$ for $1 \leq j \leq m$ denote the eigenfunctions associated to $\lambda$. Then there exist $m$ functions $t \mapsto \lambda_{k}(t), k=1, \ldots, m$, defined in a neighborhood of 0 such that

$-\lambda_{k}(0)=\lambda$,

- for every $t$ in a neighborhood of $0, \lambda_{k}(t)$ is a Steklov eigenvalue of $\Omega_{t}=(I+t \boldsymbol{V})(\Omega)$,

- the functions $t \mapsto \lambda_{k}(t), k=1, \ldots, m$, admit derivatives which are the eigenvalues of the $m \times m$ matrix $M=M_{\Omega}\left(V_{n}\right)$ of entries $\left(M_{i j}\right)$ defined by

$$
M_{j k}=\int_{\partial \Omega} V_{n}\left(-\partial_{n} u_{j} \partial_{n} u_{k}-H \lambda u_{j} u_{k}+\nabla_{\tau} u_{j} . \nabla_{\tau} u_{k}\right) d \sigma .
$$

The following result is obtain by taking $\beta \rightarrow+\infty$ in Theorem 1.4.

Theorem E.2 (Laplace-Beltrami eigenvalues). We distinguish the case of simple and multiple eigenvalue.

- If $\lambda=\lambda_{k}(\Omega)$ is a simple eigenvalue of the Laplace-Beltrami problem and $u$ an associated eigenfunction, then the application $t \rightarrow \lambda(t)=\lambda_{k}((I+t \boldsymbol{V})(\Omega))$ is differentiable and the derivative at $t=0$ is

$$
\lambda^{\prime}(0)=\int_{\partial \Omega} V_{n}\left(\left(H I_{d}-2 D^{2} b\right) \nabla_{\tau} u . \nabla_{\tau} u\right) d \sigma .
$$

The shape derivative $v^{\prime}$ of the eigenfunction satisfies

$$
\begin{aligned}
& \Delta u^{\prime}=0 \quad \text { in } \Omega, \\
& -\Delta_{\tau} u^{\prime}=\Delta_{\tau}\left(V_{n} \partial_{n} u\right)-\operatorname{div}_{\tau}\left(V_{n}\left(2 D^{2} b-H I_{d}\right) \nabla_{\tau} u\right)-\lambda^{\prime}(0) u \quad \text { on } \partial \Omega .
\end{aligned}
$$

- Let $\lambda$ be a multiple eigenvalue of order $m \geq 2$. Let $\left(u_{j}\right)$ for $1 \leq j \leq m$ denote the eigenfunctions associated to $\lambda$. Then there exists $m$ functions $t \mapsto \lambda_{k}(t), k=1, \ldots, m$, defined in a neighborhood of 0 such that

$-\lambda_{k}(0)=\lambda$,

- for every $t$ in a neighborhood of $0, \lambda_{k}(t)$ is a Laplace-Beltrami eigenvalue of $\Omega_{t}=(I+t \boldsymbol{V})(\Omega)$,

- the functions $t \mapsto \lambda_{k}(t), k=1, \ldots, m$, admit derivatives which are the eigenvalues of the $m \times m$ matrix $M=M_{\Omega}\left(V_{n}\right)$ of entries $\left(M_{i j}\right)$ defined by

$$
M_{j k}=\int_{\partial \Omega} V_{n}\left(\left(H I_{d}-2 D^{2} b\right) \nabla_{\tau} u_{i} \cdot \nabla_{\tau} u_{j}\right) d \sigma .
$$

\section{References}

[1] A. Bendali, K. Lemrabet, The effect of a thin coating on the scattering of a time-harmonic wave for the Helmholtz equation, SIAM J. Appl. Math. 56 (6) (1996) 1664-1693.

[2] M.F. Betta, F. Brock, A. Mercaldo, M.R. Posteraro, A weighted isoperimetric inequality and applications to symmetrization, J. Inequal. Appl. 4 (3) (1999) 215-240.

[3] D.D. Bleecker, The spectrum of a Riemannian manifold with a unit Killing vector field, Trans. Am. Math. Soc. 275 (1) (1983) $409-416$.

[4] V. Bonnaillie-Noël, M. Dambrine, F. Hérau, G. Vial, On generalized Ventcel's type boundary conditions for Laplace operator in a bounded domain, SIAM J. Math. Anal. 42 (2) (2010) 931-945. 
[5] L. Brasco, G. De Philippis, B. Ruffini, Spectral optimization for the Stekloff-Laplacian: the stability issue, J. Funct. Anal. 262 (11) (2012) 4675-4710.

[6] F. Brock, An isoperimetric inequality for eigenvalues of the Stekloff problem, Z. Angew. Math. Mech. 81 (1) (2001) 69-71.

[7] F. Caubet, M. Dambrine, D. Kateb, Shape optimization methods for the inverse obstacle problem with generalized impedance boundary conditions, Inverse Problems 29 (11) (2013).

[8] F.H. Clarke, Optimization and Nonsmooth Analysis, Canad. Math. Soc. Ser. Monogr. Adv. Texts, John Wiley \& Sons, Inc., New York, 1983. A Wiley-Interscience Publication.

[9] C. Cohen Tannoudji, B. Diu, F. Laloe, Mécanique Quantique, Hermann, Paris, 1997.

[10] B. Colbois, J. Dodziuk, Riemannian metrics with large $\lambda_{1}$, Proc. Am. Math. Soc. 122 (3) (1994) 905-906.

[11] B. Colbois, E.B. Dryden, A. El Soufi, Bounding the eigenvalues of the Laplace-Beltrami operator on compact submanifolds, Bull. Lond. Math. Soc. 42 (1) (2010) 96-108.

[12] M. Dambrine, D. Kateb, Persistency of wellposedness of Ventcel's boundary value problem under shape deformations, J. Math. Anal. Appl. 394 (1) (2012) 129-138.

[13] M.C. Delfour, J.-P. Zolésio, Shapes and geometries, in: Analysis, Differential Calculus, and Optimization, in: Adv. Des. Control, vol. 4, Society for Industrial and Applied Mathematics (SIAM), Philadelphia, PA, 2001.

[14] F.R. Desaint, J.-P. Zolésio, Manifold derivative in the Laplace-Beltrami equation, J. Funct. Anal. 151 (1) (1997) $234-269$.

[15] G.R. Goldstein, Derivation and physical interpretation of general boundary conditions, Adv. Differ. Equ. 11 (4) (2006) 457-480.

[16] H. Haddar, P. Joly, H.-M. Nguyen, Generalized impedance boundary conditions for scattering by strongly absorbing obstacles: the scalar case, Math. Models Methods Appl. Sci. 15 (8) (2005) 1273-1300.

[17] A. Henrot, M. Pierre, Variation et optimisation de formes, in: Une analyse géométrique (A Geometric Analysis), in: Math. Appl. (Mathematics \& Applications), vol. 48, Springer, Berlin, 2005.

[18] J. Hersch, Caractérisation variationnelle d'une somme de valeurs propres consécutives; généralisation d'inégalités de Pólya-Schiffer et de Weyl, C. R. Acad. Sci. Paris 252 (1961) 1714-1716.

[19] J. Hersch, Quatre propriétés isopérimétriques de membranes sphériques homogènes, C. R. Acad. Sci. Paris Sér. A-B 270 (1970) A1645-A1648.

[20] G.N. Hile, Z.Y. Xu, Inequalities for sums of reciprocals of eigenvalues, J. Math. Anal. Appl. 180 (2) (1993) $412-430$.

[21] J. Kennedy, A Faber-Krahn inequality for the Laplacian with generalised Wentzell boundary conditions, J. Evol. Equ. 8 (3) (2008) $557-582$.

[22] K. Lemrabet, D. Teniou, Vibrations d'une plaque mince avec raidisseur sur le bord, Maghreb Math. Rev. 2 (1) (1992) $27-41$.

[23] J.-C. Nédélec, Acoustic and electromagnetic equations, in: Integral Representations for Harmonic Problems, in: Appl. Math. Sci., vol. 144, Springer-Verlag, New York, 2001.

[24] J.H. Ortega, E. Zuazua, Generic simplicity of the eigenvalues of the Stokes system in two space dimensions, Adv. Differ. Equ. 6 (8) (2001) $987-1023$.

[25] E.M. Stein, G. Weiss, Introduction to Fourier Analysis on Euclidean Spaces, Princeton Math. Ser., vol. 32, Princeton University Press, Princeton, NJ, 1971. 